\title{
Traveling Wave Solutions in a Chain of Periodically Forced Coupled Nonlinear Oscillators
}

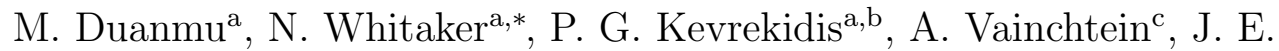 \\ Rubin ${ }^{\mathrm{c}, \mathrm{d}}$ \\ ${ }^{a}$ Department of Mathematics and Statistics, University of Massachusetts, Amherst MA \\ 01003-4515, USA \\ ${ }^{b}$ Center for Nonlinear Studies and Theoretical Division, Los Alamos National \\ Laboratory, Los Alamos, NM 87545, USA \\ ${ }^{c}$ Department of Mathematics, University of Pittsburgh, Pittsburgh, PA 15260, USA \\ ${ }^{d}$ Center for the Neural Basis of Cognition, University of Pittsburgh, Pittsburgh, PA \\ 15260, USA
}

\begin{abstract}
Motivated by earlier studies of artificial perceptions of light called phosphenes, we analyze traveling wave solutions in a chain of periodically forced coupled nonlinear oscillators modeling this phenomenon. We examine the discrete model problem in its co-traveling frame and systematically obtain the corresponding traveling waves in one spatial dimension. Direct numerical simulations as well as linear stability analysis are employed to reveal the parameter regions where the traveling waves are stable, and these waves are, in turn, connected to the standing waves analyzed in earlier work. We also consider a two-dimensional extension of the model and demonstrate the robust evolution and stability of planar fronts. Our simulations also suggest the radial fronts tend to either annihilate or expand and flatten out, depending on the phase value inside and the parameter regime. Finally, we observe that solutions that initially feature two symmetric fronts with bulged centers evolve in qualitative agreement with experimental observations of phosphenes.
\end{abstract}

Keywords:

coupled nonlinear oscillators, discrete model, traveling waves

\footnotetext{
* Corresponding author

Email address: whitaker@math.umass.edu (N. Whitaker)
} 


\section{Introduction}

Electrical stimulation of the retina can produce artificial perceptions of luminance changes called phosphenes, which may also arise in early stages of retinal or visual disease [1]. The induction of phosphenes is being used to help restore vision or develop visual aids for patients with severely compromised vision $[2,3]$, and an understanding of how phosphenes arise and behave could contribute to such efforts. In a detailed experimental study [4], Carpenter explored electrically induced phosphenes in human subjects. Each subject's eyes were immersed in a saline bath to which an alternating current was applied. When a dark object was passed through a subject's visual field in the presence of such stimulation, visual perceptions of line phosphenes occurred in its wake. The lines were observed to move and interact but never cross. This work suggested the presence of a bistability of activity states in the system, with the moving lines representing boundaries between sets of cells in different activity regimes.

Drover and Ermentrout [5] developed a one-dimensional model providing a simple representation of the phosphenes in Carpenter's experiments and their motion. In their work, a chain of excitable neurons was driven by a spatially uniform periodic stimulus at a frequency higher than the cells could follow, inducing a $1: 2$ phase locking with the stimulus. In the absence of coupling, neurons could fire on even or odd cycles of the stimulus, resulting in an intrinsic bistability for the forced system. Sufficiently strong coupling, even if directionally unbiased, elicited unidirectional traveling waves in which cells were recruited to switch phase. Large-amplitude forcing of an excitable system is a difficult problem to tackle analytically. In view of that difficulty, the more recent analysis of [6] assumed that each neuron is intrinsically oscillatory and characterized by a state evolving at half the frequency of an applied forcing signal. Using multiple time scale expansion and the Fredholm alternative, the authors of [6] derived a more analytically tractable effective model for the time evolution of the neurons' phases. This reduced model, which is quite general and particularly interesting in its own right, is the focus of the present work.

In [6], a detailed numerical existence and stability analysis was done using XPPAUT [7] for a finite chain of coupled phase oscillators. Certain interesting bifurcation phenomena were identified including saddle-node and pitchfork bifurcations in a two-dimensional parameter space characterizing the strength and asymmetry of coupling between nearest-neighbor oscilla- 
tors. Beyond the critical points involved in these bifurcations, direct numerical simulations identified traveling waves that are strongly reminiscent of the "recruitment waves" obtained in the original chain of forced neural oscillators. It is exactly these traveling waves that we systematically obtain and analyze in the present manuscript, by a combination of numerical and, whenever possible, semi-analytical techniques.

Specifically, we consider the exact traveling wave problem, which takes the form of an advance-delay differential equation in the co-traveling frame of such waves and which we introduce along with the mathematical formulation of the problem in Section 2. In Section 3, we proceed to solve this problem numerically, identifying exact (up to numerical error) traveling and standing (zero-speed) waves within the full two-dimensional parameter space used in [6]. We explore the stability of these waves in two complementary ways. On the one hand, we consider the traveling waves as steady states of the associated advance-delay partial differential equation (PDE). On the other hand, we examine them via direct numerical simulations of the original system of ordinary differential equations (ODEs) for the coupled oscillators, with the initial condition obtained from the traveling wave solution, evaluated on the lattice sites. In Section 3, as in [6], we study the problem in the physically relevant range of the parameter $\mu$ measuring the asymmetry of the nearest-neighbor coupling function but for mathematical completeness we extend the analysis to all values of $\mu$ in Appendix A. We provide strong numerical evidence, supported by the linear stability analysis of the background state presented in Appendix A, that traveling waves are stable in certain parameter regions that are periodic in $\mu$ and are located above a certain curve, below which there exist stable standing waves. The stability regions alternate along the direction of $\mu$ with regions where the waves are unstable due to the instability of the background state as well as a frontal instability. Simulations of the lattice system initialized by an unstable traveling wave show that the frontal instability results in formation of two fronts that propagate in the opposite directions with the same speed as the initial wave.

In Section 4, we extend the lattice model to a two-dimensional setting and show that the planar fronts obtained from the one-dimensional traveling wave are very robust even with a local initial distortion, unlike radial fronts. In all the case examples that we have considered, and depending on the phase value inside and the parameter regime, the latter may either be eventually annihilated or move outward and flatten out into a square-like 
shape in the dynamical evolution. Finally, we consider the evolution of two symmetric fronts with initially bulged centers and show that the resulting dynamics is in agreement with Carpenter's findings, based on observations of phosphenes, that lines form loops instead of crossing through each other and that a line does not break apart unless it meets another line. This work adds support to the idea that a phase model may provide a useful framework for studying phosphenes and also raises interesting mathematical issues about the relation between advance-delay PDE and lattice ODE system solutions, and we conclude with a brief discussion of these points in Section 5. Some details about the numerical methods can be found in Appendix B.

\section{Discrete model and the traveling wave equation}

Consider a chain of $N$ periodically forced oscillators governed by the reduced spatially discrete model of stimulated retinal cells derived in [6]:

$\dot{\theta}_{-n}=k H\left(\theta_{-n+1}-\theta_{-n}\right)+f\left(\theta_{-n}\right)$

$\dot{\theta}_{j}=k\left[H\left(\theta_{j-1}-\theta_{j}\right)+H\left(\theta_{j+1}-\theta_{j}\right)\right]+f\left(\theta_{j}\right)$, for $j=-n+1, \ldots, N-n-2$

$\dot{\theta}_{N-n-1}=k H\left(\theta_{N-n-2}-\theta_{N-n-1}\right)+f\left(\theta_{N-n-1}\right)$.

Here $\theta_{j}(t)$ is the slowly evolving phase of each neuron, $f(\theta)$ is a $\pi$-periodic forcing or locking function, and $H(\theta)$ is a $2 \pi$-periodic function characterizing the coupling of the nearest neighbors and multiplied by the coupling constant $k>0$. The periodicities of $f(\theta)$ and $H(\theta)$ represent the $1: 2$ frequency locking present in the system and can be easily generalized to different types of frequency locking. In [6] the number of oscillators was set to be even, $N=2 n$; here, we also also allow it to be odd, with $N \in\{2 n, 2 n+1\}$.

In what follows, we consider prototypical examples of the two functions proposed in [6],

$$
H(\theta)=\sin (\theta+\mu)-\sin (\mu), \quad f(\theta)=-\sin (2 \theta),
$$

where the parameter $\mu$ measures the asymmetry of the coupling function $H(\theta)$. From a biological perspective, it makes sense to identify 0 with $2 \pi$ such that $\theta_{j} \in[0,2 \pi)$ and then the firing of the $j$ th neuron corresponds to $\theta_{j}$ crossing through some distinguished value, taken for some oscillator models, for example, to be $\theta_{j}=\pi$; for plotting purposes in this paper, however, it 
is sometimes convenient to treat $\theta_{j}$ as varying in $\mathbb{R}$. It is not hard to see that in the case of $\mu=0$, when $H(\theta)=\sin \theta$ is odd, there exist equilibrium split-state (antiphase) solutions given by

$\left(\theta_{-n}, \ldots, \theta_{-1}, \theta_{0}, \ldots, \theta_{N-n-1}\right)=(0, \ldots, 0, \pi, \ldots, \pi) \quad$ and $\quad(\pi, \ldots, \pi, 0, \ldots, 0)$.

If $N=2 n$, these solutions have equal numbers of oscillators firing in each cycle. When $\mu$ is nonzero, such piecewise constant split states no longer exist. However, there are single-front equilibrium split states that are close to the above antiphase state but have boundary layers near the front. In [6], such steady-state solutions of (1) are obtained numerically by varying $\mu$ and $k$. A prototypical example of the corresponding bifurcation diagram in the $(\mu, k)$ plane is shown in Fig. 1, adapted from Fig. 4 in [6]. There we observe that the primary split-state solution is stable in a region of small enough $k$ (region A) but becomes unstable through a pitchfork bifurcation at $k=k^{*}(\mu)$ shown by the solid curve. This bifurcation is subcritical for $\mu$ above a certain threshold, $\mu>\mu_{c}$. For $\mu<\mu_{c}$, the bifurcation is supercritical and gives rise to two other, secondary, stable nonsymmetric split states that exist in region B. These states, in turn, disappear through a pair of saddle-node bifurcations at $k=k^{* *}(\mu)$ (dashed curve), where $k^{* *}(\mu)>k^{*}(\mu)$ for $\mu<\mu_{c}$ and $k^{* *}\left(\mu_{c}\right)=k^{*}\left(\mu_{c}\right)$, corresponding to a codimension-2 point $\left(\mu_{c}, k^{*}\left(\mu_{c}\right)\right)$ where the saddle-node curves emerge and the pitchfork bifurcation changes criticality. When exploring the dynamical byproducts of these instabilities, the authors of [6] identified a spontaneous emergence of traveling waves in region C. This motivates us to seek (numerically) exact traveling wave solutions for this problem.

To this end, we consider the infinite chain of oscillators

$$
\dot{\theta}_{j}=k\left[H\left(\theta_{j-1}-\theta_{j}\right)+H\left(\theta_{j+1}-\theta_{j}\right)\right]+f\left(\theta_{j}\right), \quad j \in \mathbb{Z} .
$$

We start by seeking a solution of this system in the form $\theta_{j}(t)=\Theta(z, \tau)$, where $z=j-c t$ is a traveling wave coordinate with wave velocity $c$ and $\tau=t$, such that $\Theta \rightarrow 0$ and $\Theta \rightarrow \pi$ when $z \rightarrow-\infty$ and $\infty$, respectively, or vice versa. Substitution of this ansatz into (2) leads to the partial differential advance-delay equation (co-traveling frame PDE) of the form

$$
\begin{aligned}
\Theta_{\tau}-c \Theta_{z}= & k[H(\Theta(z+1, \tau)-\Theta(z, \tau))+H(\Theta(z-1, \tau)-\Theta(z, \tau))] \\
& +f(\Theta(z, \tau)) .
\end{aligned}
$$




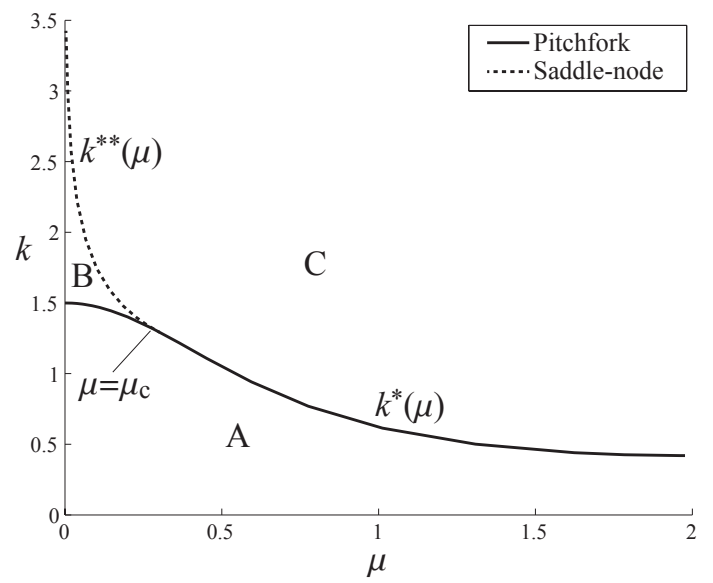

Figure 1: Bifurcation diagram for stationary solutions of the system (1) with $N=2 n=$ 10 , as obtained in [6]. The primary split-state equilibria are stable in region $\mathrm{A}$ and destabilize via a pitchfork bifurcation (solid curve, $k=k^{*}(\mu)$ ). At $\mu<\mu_{c}$ the bifurcation is supercritical and gives rise to a pair of secondary split-state equilibria that are stable in region $\mathrm{B}$ and disappear through a saddle-node bifurcation (dashed curve, $k=k^{* *}(\mu)$, $\left.\mu<\mu_{c}\right)$.

Traveling wave solutions of (2),

$$
\theta_{j}(t)=\phi(z), \quad z=j-c t
$$

are stationary solutions of (3) and satisfy the advance-delay differential equation

$$
-c \phi^{\prime}(z)=k[H(\phi(z+1)-\phi(z))+H(\phi(z-1)-\phi(z))]+f(\phi(z)) .
$$

Since every translate of the traveling solution is also a solution, the additional pinning condition $\phi(0)=\pi / 2$ is imposed on the nonlinear system in order to fix (i.e., pin) the traveling wave. This condition uniquely identifies the solution, as well as the corresponding velocity $c$. The solution $\phi(z)$ contains all of the information about the traveling wave solution of the ODE system (2): for any integer $j, \theta_{j}(0)=\phi(j)$ at $t=0$ and $\theta_{j}(t)=\phi(j-c t)$ at $t>0$. Note that the traveling wave ansatz (4) implies that $\theta_{j}(t)=\theta_{j+1}(t+1 / c)$ for any integer $j$, meaning that the phase value $\theta_{j}(t)$ is periodic (modulo shifts) with a period of $1 / c$. 


\section{Existence, Stability and Dynamics}

\subsection{Examples of traveling wave solutions}

We start by considering solutions of the traveling wave equation (5) satisfying $\phi(0)=\pi / 2, \phi(z) \rightarrow \pi$ as $z \rightarrow \infty, \phi(z) \rightarrow 0$ as $z \rightarrow-\infty$. Eq. (5) is discretized in the interval $[-25,25]$ with a forward difference approximation of the derivative in the left hand side (for comparison purposes, a centered difference scheme was also used) and $\Delta z=0.025$. The middle grid point is fixed to satisfy the pinning condition $\phi(0)=\pi / 2$, and the speed $c$ is added as a variable. We solve the resulting system using Newton's method for the traveling wave $\phi(z)$ and its velocity $c$, thus identifying numerically exact (at least up to the prescribed tolerance of $10^{-12}$ in the discretized system) solutions.

Fig. 2(a-c) shows examples of traveling wave solutions corresponding to different values of the parameters $k$ and $\mu$ that characterize the intersite coupling. In each case, stability of the traveling wave solution $\phi(z)$ is analyzed by computing the spectrum of the Jacobian matrix obtained by linearizing Eq. (3) about $\phi(z)$ with a forward difference approximation of the spatial derivative (see Appendix B for further details); the resulting spectra are shown in Fig. 2(d-f). We also check stability of the obtained solutions by solving the ODE system (1) initialized at the traveling wave. For example, in Fig. 2(g-i), we show the space-time evolution of the solution of the ODE system (1) with $N=2 n+1=51$ oscillators initialized by the traveling wave solution $\phi(z)$ evaluated at the integer values of $z, \theta_{j}(0)=\phi(j)$, $j=-25, \ldots, 25$. The time evolution of the ODE system (1) is accomplished using the classical fourth-order Runge-Kutta method.

In Fig. 2(a), the traveling wave solution $\phi(z)$ (solid line) is shown at $k=2.25$ and $\mu=0.5$, which yields $c=0.8123$. Fig. $2(\mathrm{~d})$ indicates that the eigenvalues of the linearization Jacobian of the PDE (3), evaluated at this solution, have only negative real parts, implying the linear (spectral) stability of the traveling wave solution as a stationary solution of Eq. (3). Finally, Fig. 2(g) shows the evolution of the solution of the discrete ODE system (1) initialized by the traveling wave solution, $\theta_{j}(0)=\phi(j)$. As anticipated by the traveling wave ansatz, the ODE solution is found to traverse the lattice at the velocity $c=0.8124$, which agrees with the traveling wave velocity very well ${ }^{1}$. Further evidence that the traveling wave solution indeed represents the

\footnotetext{
${ }^{1}$ The speed of the front is numerically calculated as follows. We choose an integer grid
} 


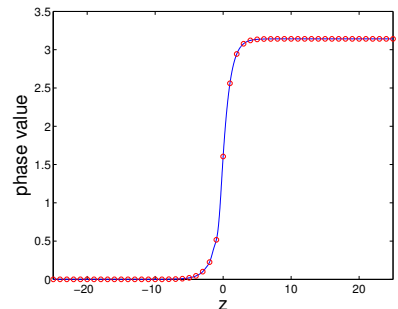

(a)

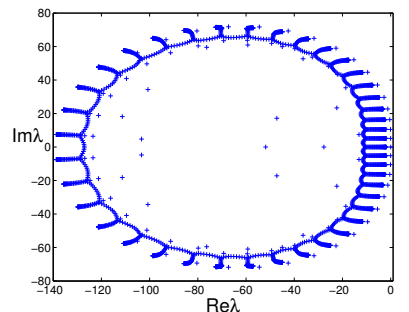

(d)

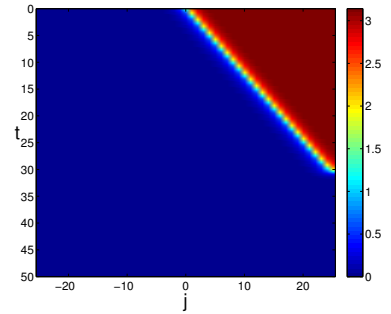

$(\mathrm{g})$

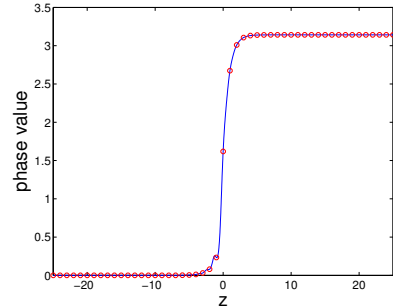

(b)

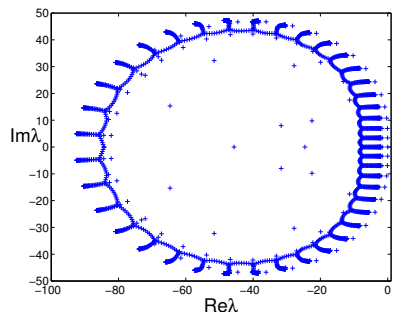

(e)

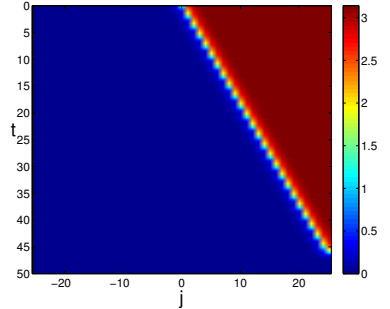

(h)

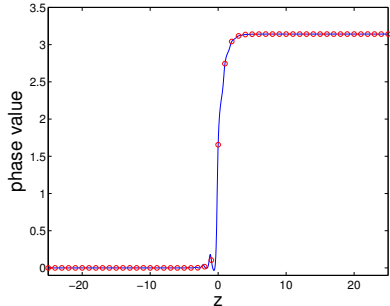

(c)

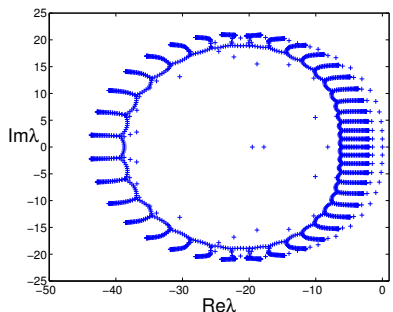

(f)

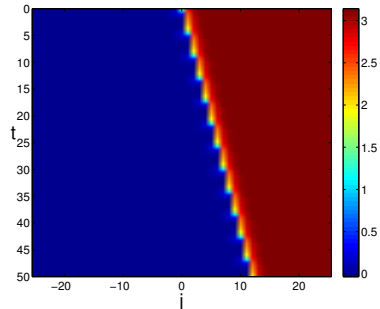

(i)

Figure 2: Top row: traveling wave solution (solid curve) $\phi(z)$ of (5) at $\mu=0.5$ and (a) $k=2.25$, (b) $k=1.5$ and (c) $k=1.1$, yielding $c=0.8123,0.5368$ and 0.2382 respectively, and the initial condition (circles) $\theta_{j}(0)=\phi(j)$ used for the ODE simulations shown in $(\mathrm{g}),(\mathrm{h})$ and (i). Middle row: eigenvalues of the Jacobian of the linearization of the cotraveling frame PDE (3) around the stationary solution $\phi(z)$. Bottom row: space-time evolution of the solution of ODE (1) with initial condition $\theta_{j}(0)=\phi(j)$ shown by circles in (a),(b) and (c), respectively.

point $k$ and place the right-traveling front to its left, near an integer grid point $m$ with $m<k$ such that $\left|\theta_{m}-\frac{\pi}{2}\right|<\left|\theta_{j}-\frac{\pi}{2}\right|$ for all $j$. The front is allowed to evolve using the standard fourth order Runge-Kutta method. We count the number of time steps $p$ such that $\left|\theta_{k}-\frac{\pi}{2}\right|<\left|\theta_{j}-\frac{\pi}{2}\right|$ for all $j$ and approximate the speed by $\frac{1}{p \Delta t}$ where $\Delta t$ is the Runge-Kutta time step. The error is observed to be $O(\Delta t)$. If $p=0$, meaning that the 
dynamics of the ODE system can be seen in Fig. 3(a), where one can observe an excellent agreement between $\theta_{20}(t)$ plotted as a function of $z=20-c t$ and the traveling wave solution $\phi(z)$ of $(5)$. Observe that the traveling wave profile $\phi(z)$ is not monotone and exhibits oscillations behind the front. To the best of our understanding, these oscillations are not a numerical artifact since they persist even as the grid is refined and are also captured by the ODE dynamics (1). While we cannot fully explain their nature, we suspect that they arise due to the nonlinearity of the coupling function $H(\theta)$ because linear coupling is associated with monotone profiles [8]. It may be worthwhile to further explore this issue in the future work.

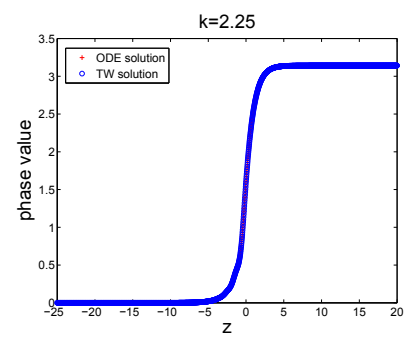

(a)

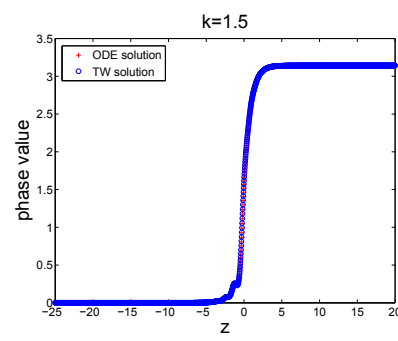

(b)

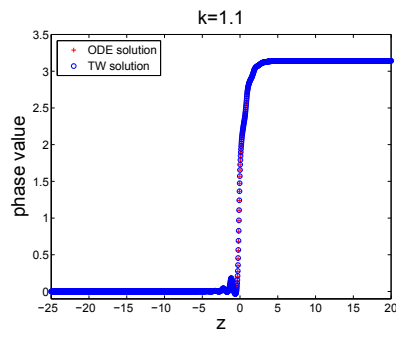

(c)

Figure 3: Comparison of the solution $\theta_{20}(t)$ (pluses) of the ODE system (1), plotted as a function of $z=20-c t$, and the traveling wave solution $\phi(z)$ of $(5)$ at $\mu=0.5$ and (a) $k=2.25$, (b) $k=1.5$ and (c) $k=1.1$. For comparison purposes the time step used to solve (1) was set to $\Delta t=\Delta z / c$, where $\Delta z$ is the mesh size in the numerical solution of (5) and $c$ is the obtained speed of the traveling wave.

Fig. 2(b,e,h) and Fig. 2(c,f,i) illustrate the cases of $k=1.5$ and $k=1.1$ at $\mu=0.5$, with traveling wave velocities $c=0.5368$ and $c=0.2382$, respectively, obtained by solving Eq. (5). These are very close to the velocities $c=0.5367$ and $c=0.2377$, respectively, obtained from the solution of the ODE system (1) initialized by the traveling wave (Fig. 2, bottom row). As before, direct comparison of the ODE dynamics and the traveling wave solution in Fig. 3(b,c) shows a very good agreement. Observe that as $k$ decreases at fixed $\mu$, the velocity $c$ of the traveling wave decreases, and the eigenvalues shown in Fig. 2(d-f) move closer to the imaginary axis as well. Nonethe-

front never entered the interval $\left[k-\frac{1}{2}, k+\frac{1}{2}\right]$, then the speed is zero, assuming that the method was allowed to run long enough. 


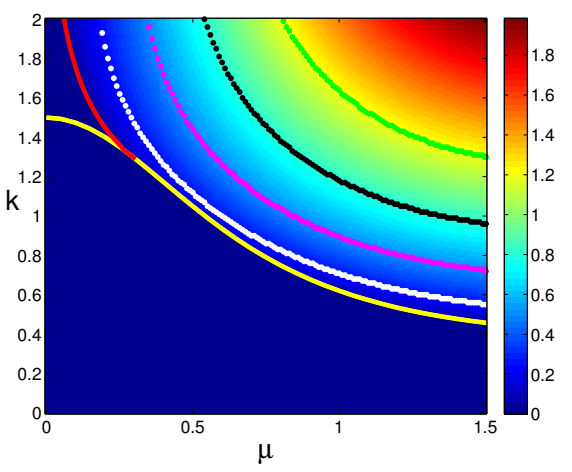

(a)

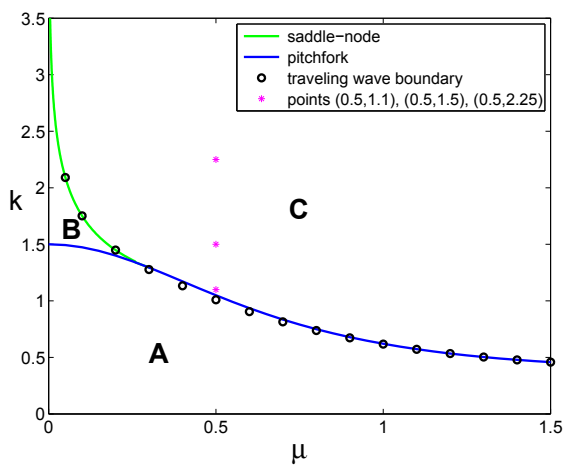

(b)

Figure 4: (a) Density plot of the velocity $c$ of the traveling waves in the $(\mu, k)$ plane obtained by solving Eq. (5) and isocontours of $c=0.25$ (white), $c=0.5$ (magenta), $c=0.8$ (black) and $c=1.2$ (green), shown together with the pitchfork (yellow) and saddle-node (red) bifurcation curves obtained for the equilibrium states of the 51-oscillator chain. (b) The curve (open circles) where the traveling waves have (almost) zero velocity nearly coincides with the saddle-node (green) and pitchfork (blue) bifurcation curves; parameter values used in Fig. 2 are also shown (magenta stars).

less, the obtained eigenvalues in all three cases remain in the left half-plane $\operatorname{Re} \lambda<0$, suggesting that the relevant waves are stable as stationary solutions of the co-traveling wave PDE (3). We anticipate that this spectral stability of the solution for the PDE implies the dynamical stability of the traveling wave as the solution of the ODE system (1), as confirmed by our simulations. However, the general demonstration of such a connection is, to the best of our knowledge, an intriguing open problem in analysis.

\subsection{Existence and stability of traveling waves for the chain}

We now explore more systematically the existence and stability of traveling waves in the $(\mu, k)$ parameter plane. The existence results for sufficiently small $\mu(0 \leq \mu \leq 1.5)$ are summarized in Fig. 4(a), which shows the density plot of velocity of the traveling wave computed at different $\mu$ and $k$ using Eq. (5) and complements the steady state analysis of [6] shown in Fig. 1. To obtain this plot, the forward-difference discretization of Eq. (5) with $\Delta z=0.025$ is solved on $[-25,25]$ using Newton's method as described above for each $\left(\mu_{i}, k_{j}\right)$, where $\mu_{i}=i \Delta \mu, i=0, \ldots, 151$, and $k_{j}=j \Delta k$, $k=0, \ldots, 201$, with $\Delta \mu=\Delta k=0.01$. To further illustrate how the veloc- 
ity $c$ of traveling wave depends on $\mu$ and $k$, we also include the isocontours associated with different velocities. For comparison, pitchfork (yellow) and saddle-node (red) bifurcation curves for the 51-oscillator chain (shown by solid and dashed curves, respectively, in Fig. 1) are also included. To obtain the pitchfork curve, stationary solutions of Eq. (1) are computed using Newton's method, starting with the value $k=0.4$ for given $\mu$ and using the continuation procedure with increasing $k$ until the Jacobian of the relevant system becomes singular. To obtain the saddle-node curve, a stable stationary solution is identified in region B of Fig. 1. This is accomplished by integrating Eq. (1) with an unstable solution as an initial condition over a long time horizon until a stable stationary solution is reached as an attractor of the system's dynamics. This solution is used as the initial guess in the Newton's method to obtain the stable equilibrium state within the region B. Numerical continuation is then performed with increasing $\mu$ until the Jacobian becomes singular. This monoparametric process is repeated for different values of $k$ within region $\mathrm{B}$. The obtained curves are almost indistinguishable from the ones in Fig. 1. While we display the results of our computations on a specific finite chain, having examined chains of different sizes, we expect our principal conclusions to persist (essentially without modification) for the case of the infinite chain. On the flip side, we can identify traveling waves only in region $\mathrm{C}$, while below it their speed degenerates to zero, leading to standing waves (split-state equilibria), in agreement with the findings of [6] for the finite chain case (and our discussion above of standing wave states).

We explore this comparison in more quantitative detail in Fig. 4(b). Here the open circles mark the curve above which numerical simulations of ODE system (1) yield stable traveling waves. To obtain these points, we fixed $\mu$ and solved (5) for the traveling wave solution $\phi(z)$ and its velocity at $(\mu, k)$ starting with $k$ just above the bifurcation curves and then progressively decreasing its value. To verify the velocity of the traveling wave at given $(\mu, k)$, we then used $\theta_{j}(0)=\phi(j)$ as initial conditions in the simulations of (1) and computed the velocity of the propagating front. The open circles were obtained by finding the values of $k$ where the traveling wave solution has zero velocity up to the numerical error in both methods. The comparison strongly suggests that the disappearance of standing wave solutions of [6] gives rise to the traveling wave solutions analyzed here.

To further illustrate the bifurcations in Fig. 4(b), we include Fig. 5(a), which shows the bifurcation diagrams in the $\left(k, \theta_{-1}\right)$ plane, where $\theta_{-1}$ is the phase of the 25 th oscillator in the 50 -oscillator chain, at $\mu=0.1$, below 


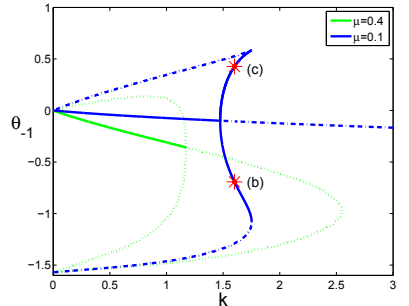

(a)

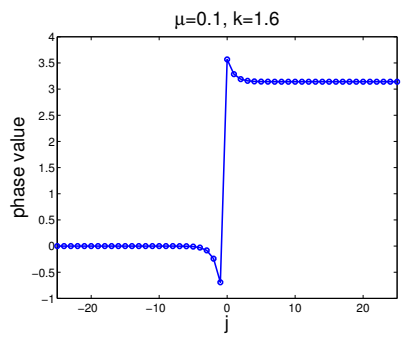

(b)

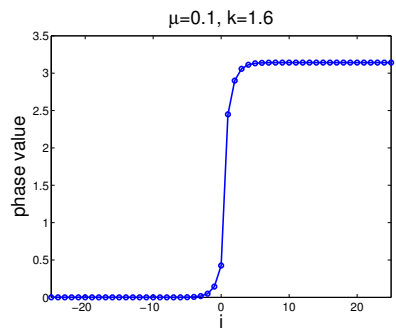

(c)

Figure 5: (a) Supercritical pitchfork and saddle-node bifurcations at $\mu=0.1<\mu_{c}$ and subcritical pitchfork bifurcation at $\mu=0.4>\mu_{c}$ for a 50-oscillator system. Solid curves correspond to stable solutions, and dashed to unstable ones. Two different stable solutions for $\mu=0.1$ and $k=1.6$, marked by stars in (a), are shown in (b) and (c).

the critical value $\mu_{c}$ where the saddle-node and pitchfork bifurcation curves merge, and $\mu=0.4$, above this value. At $\mu=0.1<\mu_{c}$ one can see a supercritical pitchfork bifurcation that gives rise to two stable split-state equilibria existing for a range of the parameter $k$. The panels (b) and (c) in Fig. 5 show these solutions at $k=1.6$, with the corresponding points marked by stars in panel (a). At large enough $k$ these equilibria merge with unstable ones and disappear through saddle-node bifurcations, so that for higher $k$ there are no stable equilibria. Meanwhile, at $\mu=0.4>\mu_{c}$ the pitchfork bifurcation becomes subcritical (note that there is also a saddlenode bifurcation at larger $k$ where two unstable equilibria merge). Similar diagrams were obtained in [6] for a 10-oscillator system.

Using symmetry and periodicity of the functions $H(\theta)$ and $f(\theta)$, one can extend the bifurcation diagram in Fig 4(b) to the entire real axis in $\mu$; see Appendix A for details. In particular, this diagram has even symmetry about the vertical axis, as shown in Fig. A.16. In Appendix A, we also analytically compute the spectra of the background states $\Theta=0$ and $\Theta=\pi$. Henceforth, in the main text, we focus on the physically meaningful range $|\mu|<\pi / 2$, which ensures that the nearest-neighbor coupling is attractive for small enough $\theta$ and that the spectra of the background states are confined to the negative real part of the complex plane, ruling out background instability (Appendix A).

Our results suggest that at any $(\mu, k)$ in the region $\mathrm{C}$, with $0<\mu<$ $\pi / 2$, there exists a traveling wave solution $\phi(z)$ with velocity $c>0$ that satisfies $\lim _{z \rightarrow \infty} \phi(z)=\pi$ and $\lim _{z \rightarrow-\infty} \phi(z)=0$. By reflectional symmetry, 

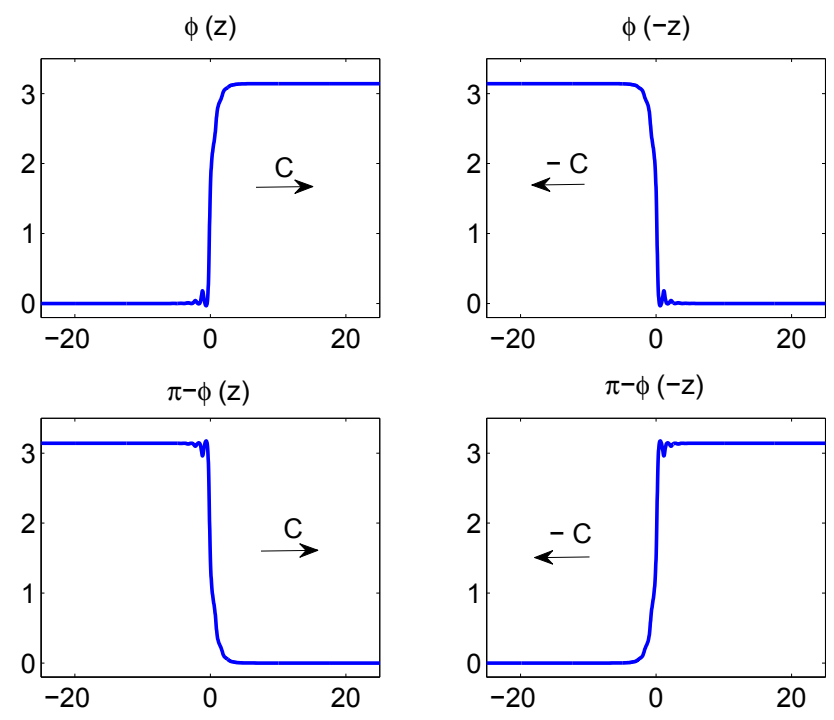

Figure 6: Symmetric traveling wave solutions at $(\mu, k)$ (upper panels) and $(-\mu, k)$ (lower panels), for $0<\mu<\pi / 2$.

there is also the solution $\bar{\phi}(z)=\phi(-z)$ with velocity $\bar{c}=-c<0$ that satisfies $\lim _{z \rightarrow \infty} \bar{\phi}(z)=0$ and $\lim _{z \rightarrow-\infty} \bar{\phi}(z)=\pi$. Both solutions involve the phase value $\theta=0$ displacing $\theta=\pi$ as the front propagates. As shown in Appendix A, by symmetry and periodicity of $H(\theta)$ and $f(\theta)$, at $(-\mu, k)$ there are traveling wave solutions $\pi-\phi(z)$, with velocity $c$, and $\pi-\phi(-z)$, with velocity $-c$, where $\theta=\pi$ displaces $\theta=0$; see Fig. 6 and Table A.1 in Appendix A. In summary, for the traveling waves at $0<\mu<\pi / 2$, the phase value 0 always displaces $\pi$ and for waves at $-\pi / 2<\mu<0, \pi$ displaces 0 . This observation will be used to interpret some results for the two-dimensional problem considered in the next section.

\section{Two-dimensional phase equation dynamics}

We now explore a two-dimensional generalization of our oscillator problem. Specifically, we consider the following extension of Eq. (1) to two di- 
mensions (for a square lattice of $2 m \times 2 n$ oscillators):

$$
\begin{aligned}
& \dot{\theta}_{-m,-n}=k\left(H\left(\theta_{-m,-n+1}-\theta_{-m,-n}\right)+H\left(\theta_{-m+1,-n}-\theta_{-m,-n}\right)\right)+f\left(\theta_{-m,-n}\right) \\
& \dot{\theta}_{i,-n}=k\left(H\left(\theta_{i,-n+1}-\theta_{i,-n}\right)+H\left(\theta_{i+1,-n}-\theta_{i,-n}\right)+H\left(\theta_{i-1,-n}-\theta_{i,-n}\right)\right) \\
& \quad+f\left(\theta_{i,-n}\right), \quad i=-m+1, \ldots, m-2, \\
& \dot{\theta}_{-m, j}=k\left(H\left(\theta_{-m, j+1}-\theta_{-m, j}\right)+H\left(\theta_{-m, j-1}-\theta_{-m, j}\right)+H\left(\theta_{-m+1, j}-\theta_{-m, j}\right)\right) \\
& \quad+f\left(\theta_{-m, j}\right), \quad j=-n+1, \ldots, n-2, \\
& \dot{\theta}_{i, j}=k\left(H\left(\theta_{i, j+1}-\theta_{i, j}\right)+H\left(\theta_{i, j-1}-\theta_{i, j}\right)+H\left(\theta_{i+1, j}-\theta_{i, j}\right)+H\left(\theta_{i-1, j}-\theta_{i, j}\right)\right) \\
& \quad+f\left(\theta_{i, j}\right), \quad i=-m+1, \ldots, m-2, \quad j=-n+1, \ldots, n-2, \\
& \quad \dot{\theta}_{i, n-1}=k\left(H\left(\theta_{i, n-2}-\theta_{i, n-1}\right)+H\left(\theta_{i+1, n-1}-\theta_{i, n-1}\right)+H\left(\theta_{i-1, n-1}-\theta_{i, n-1}\right)\right) \\
& \quad+f\left(\theta_{i, n-1}\right), \quad i=-m+1, \ldots, m-2, \\
& \dot{\theta}_{m-1, j}=k\left(H\left(\theta_{m-1, j+1}-\theta_{m-1, j}\right)+H\left(\theta_{m-1, j-1}-\theta_{m-1, j}\right)+H\left(\theta_{m-2, j}-\theta_{m-1, j}\right)\right) \\
& \quad+f\left(\theta_{m-1, j}\right), \quad j=-n+1, \ldots, n-2, \\
& \dot{\theta}_{m-1, n-1}=k\left(H\left(\theta_{m-1, n-2}-\theta_{m-1, n-1}\right)+H\left(\theta_{m-2, n-1}-\theta_{m-1, n-1}\right)\right)+f\left(\theta_{m-1, n-1}\right) .
\end{aligned}
$$

We start by solving Eq. (5) on the interval $[-25,25]$ at the parameter values $k=1.3$ and $\mu=0.5$ to obtain a one-dimensional traveling wave $\phi(z)$ with velocity $c=0.4155$. We then solve Eq. (6) for $\theta_{i, j}(t)$ using the classical fourth order Runge-Kutta method with the initial data

$$
\theta_{i, j}(0)= \begin{cases}0, & i \leq-247 \\ \phi(i+247), & -246 \leq i \leq-196 \\ \pi, & i \geq-196\end{cases}
$$

thus studying the two-dimensional evolution of an initially planar front. The planar front propagates in the horizontal direction at the velocity of the one-dimensional wave, suggesting that the solution $\theta_{i, j}(t)=\phi(i-c t)$, which corresponds to a stable traveling wave in the one-dimensional problem, is also stable in the two-dimensional setting. To illustrate this stability, we distort a segment of the planar front in the initial condition, as shown in the first panel $(t=0)$ in Fig. 7 . The resulting evolution is shown in the remaining panels of Fig. 7, where for better visualization, the range of the $i$-axis is shifted to the left in the bottom three panels. It can be seen that over time the system "heals" the perturbation and gradually restores its quasi-onedimensional planar front character, while the solution eventually settles into 
traveling with the velocity predicted by the one-dimensional results. Here $0<\mu<\pi / 2$, and we have $\theta=0$ displacing $\theta=\pi$ as the front propagates, as discussed at the end of the previous section. For $k=1.3, \mu=-0.5$, the front
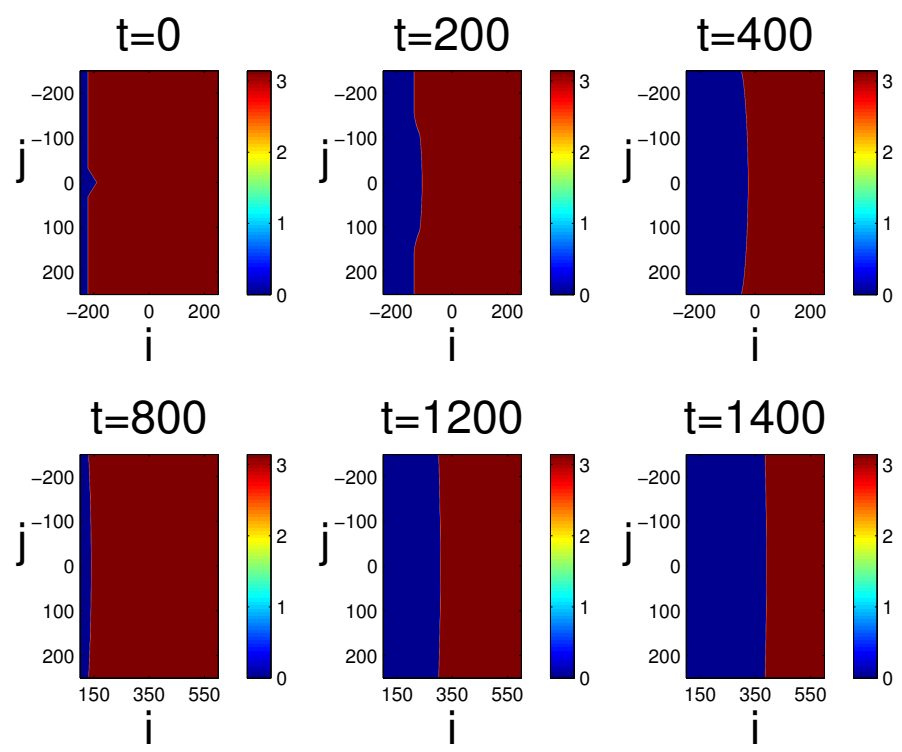

Figure 7: Snapshots of the evolution of a perturbed planar front introduced within a twodimensional domain with $k=1.3, \mu=0.5$ at times $t=0,200,400,800,1200$ and 1400 . Color coding indicates values of $\theta_{i, j}$. The evolution shows the healing of the front and the decay of the associated perturbation that restore the dynamically robust planar front traveling with velocity $c=0.4155$.

(due to symmetry) moves in the opposite direction with the same speed.

Next we consider the evolution of a radial front. In the first four panels of Fig. 8, we show the evolution of an initially circular front for $k=1.3$ and $\mu=0.5$. The initial condition is set to $\theta_{i, j}(0)=\pi$ for $(i, j)$ within the circle of radius 30 centered at $(0,0)$ and $\theta_{i, j}(0)=0$ outside of the concentric circle of radius 36 . The initial value of $\theta_{i, j}$ for $(i, j)$ between these circles is obtained by linear interpolation. The front shrinks and is eventually annihilated. As the front evolves, $\theta=0$ again displaces $\theta=\pi$, which is expected since $0<\mu<$ $\pi / 2$. Notice that, apparently, the initial radial profile of the front is gradually deformed to conform more suitably to the symmetry of the underlying square lattice. The bottom panel of Fig. 8 displays horizontal slices of the solution 
at the initial and final time steps shown above.

In Fig. 9, we consider the same parameter values $\mu=0.5$ and $k=1.3$ but in the initial condition the oscillator takes the value 0 inside the inner circle and $\pi$ outside the outer circle. As in the previous figure, the phase value 0 displaces $\pi$ as the front propagates but now it evolves outward. In Fig. 10, the initial condition has phase value $\pi$ inside the inner circle and zero outside the outer one, as in Fig. 8 , but now $\mu=-0.5$ and $k=1.3$. In this case the front moves outward since $-\pi / 2<\mu<0$ and the phase $\theta=\pi$ displaces zero. In both simulations the outward propagation of the front is once again accompanied by its flattening out into a square-like shape due to the symmetry of the underlying lattice. In all initial data used in our 2 -d simulations, the region with the 0 oscillators is always connected to the region with the $\pi$ oscillators through linear interpolation. If the connection is not smooth enough then instabilities arise near the transition region.

As noted in [5], Carpenter [4] observed that experimentally-induced phosphenes move according to the following rules in two dimensions:

1. Lines never cross through one another. Rather, they combine to form loops.

2. A line never breaks apart unless it meets another line.

To test whether our two-dimensional model captures these features, we now consider simulations with two symmetric fronts that initially bulge either outward (Fig. 11 and Fig. 13) or inward (Fig. 12 and Fig. 14). These initial conditions are shown in the first panel $(t=0)$ of each figure. The simulation results shown in Figs. 11-14, with $k=1.3, \mu=0.5$ in Fig. 11 and Fig. 12 and $k=2, \mu=0.15$ in Fig. 13 and Fig. 14, are consistent with Carpenter's observations listed above. We can see that the outwardly bulging fronts eventually touch near the top and bottom edges of the domain and form one loop. Meanwhile, the inwardly bulging fronts eventually touch near their centers and the lines break into two parts. These features are very much in line with the expectations of [5] (compare with their Fig. 3). However, it should also be mentioned that [5] posits a third and final feature, namely that:

3. Neighboring lines show a tendency to move in a similar manner. Our simulations of the coupled oscillator model did not reveal such a tendency. How the model can be improved to reflect this feature is a question that remains to be considered in future studies. 

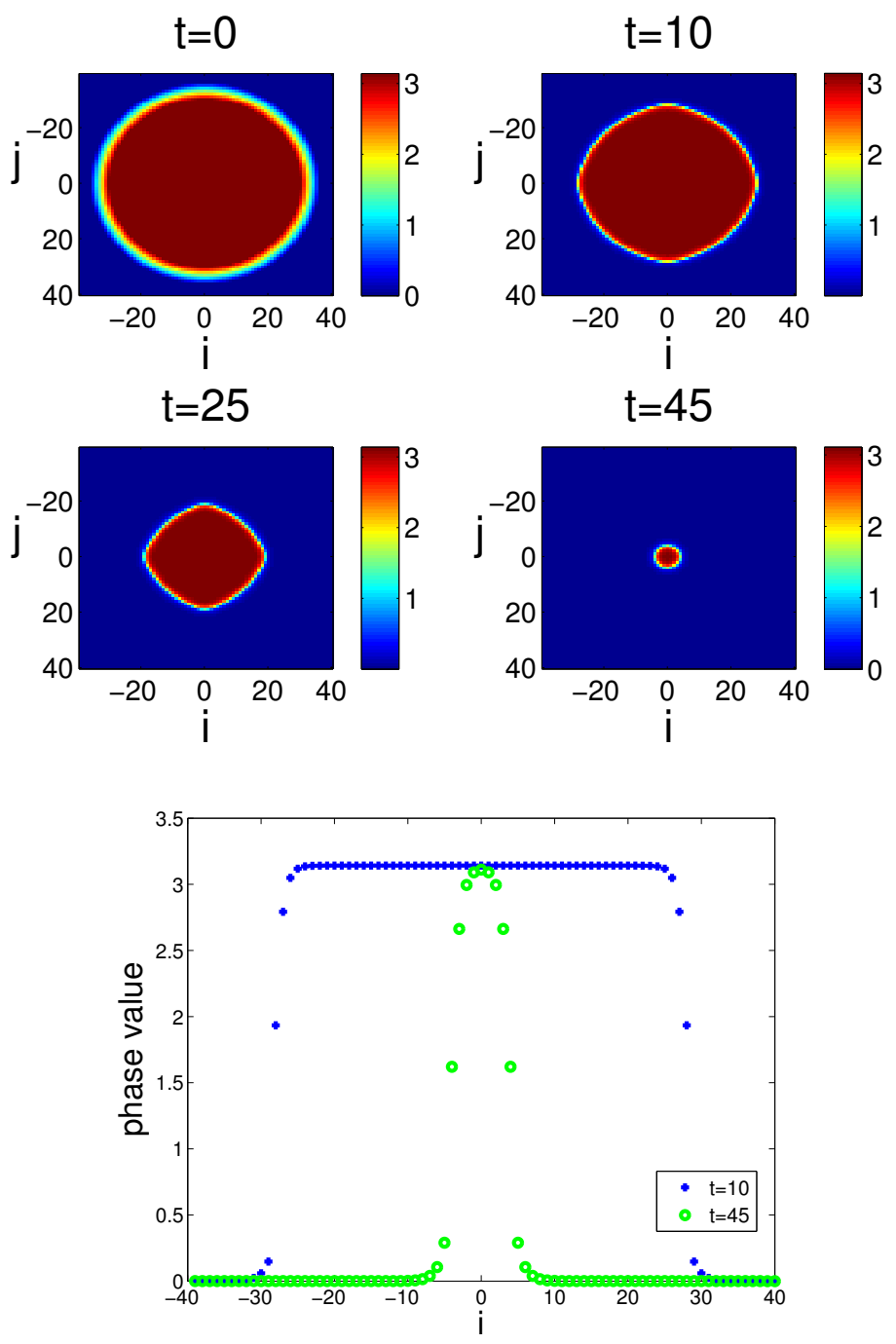

Figure 8: Snapshots of the evolution of a circular front with $k=1.3, \mu=0.5$ at times $t=0,10,25$ and 45. Inside the circle the oscillators' value is $\pi$ and outside the circle it is 0 . The front shrinks (and is eventually annihilated) as time evolves. In the bottom panel, a horizontal section $(j=0)$ of the front at $t=10$ is denoted by plus signs and a horizontal section of the front at $t=45$ (of smaller width) is denoted by circles. 

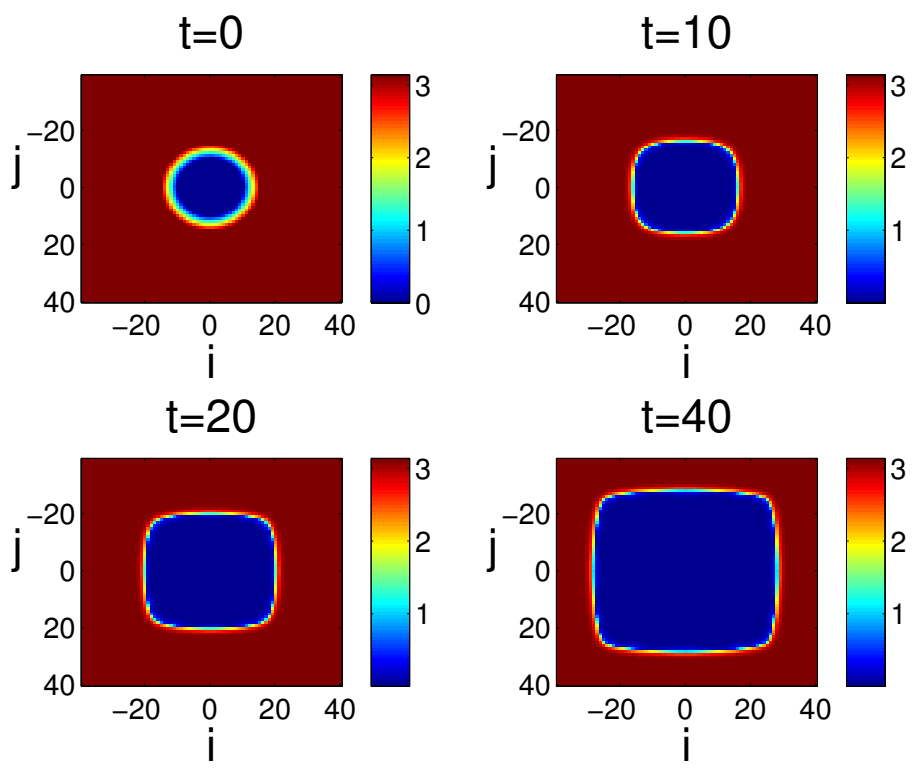

Figure 9: Time evolution at $\mu=0.5, k=1.3$. The initial condition is set to $\theta_{i, j}=\pi$ outside the circle of radius 15 and to zero inside the concentric circle of radius 10 , with linear interpolation between the two circles. The front moves outward.

\section{Conclusions and Future Challenges}

In the present work we have revisited a generic nonlinear lattice model derived in [6] and associated with the dynamics of a forced network of coupled oscillators. A specific motivation for studying this system comes from its relevance to phosphenes, artificial perceptions of light arising in the visual system in which contours, possibly representing boundaries between sets of neurons in different activity states or phases, emerge and propagate. We complemented the important initial steady-state analysis of [6] (see also [5]) by exploring the possibility of traveling waves in the system of ordinary differential equations. This led us to introduce the co-traveling frame (advancedelay) PDE and study existence and stability properties of the traveling waves as both special periodic modulo shift solutions of the original system of ODEs and stationary solutions of the co-traveling frame PDE. Our results on traveling waves complement the work of [6] on the existence and stability of standing waves. We found a curve above which the standing waves become 

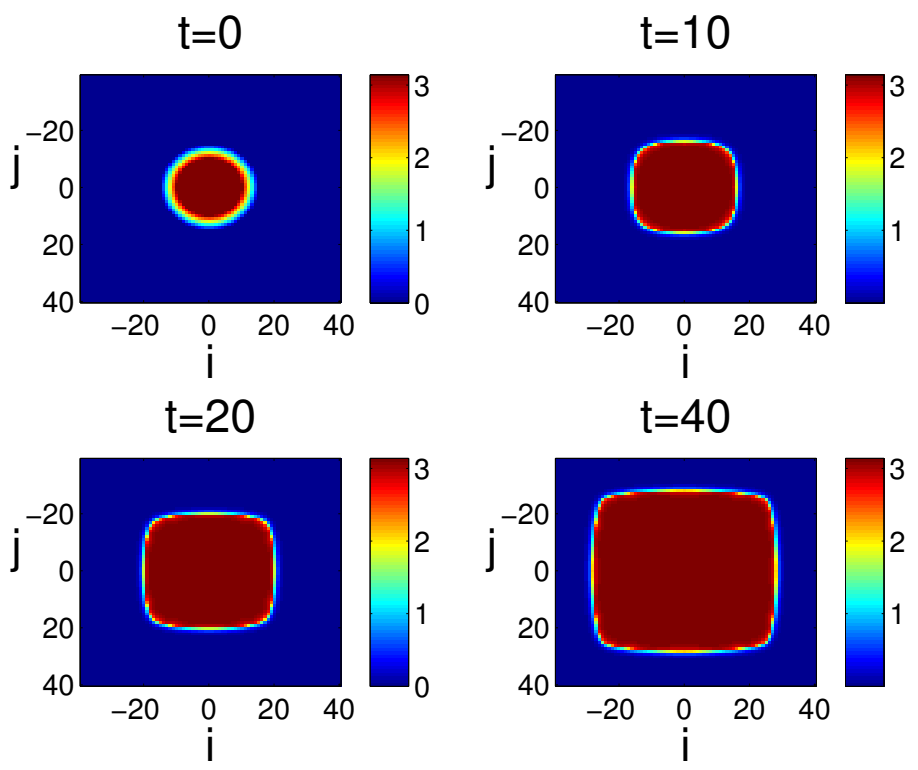

Figure 10: Time evolution at $\mu=-0.5, k=1.3$. The initial condition is set to $\theta_{i, j}=\pi$ inside the circle of radius 10 and to zero outside the concentric circle of radius 15 , with linear interpolation between the two circles. The front moves outward.

unstable. This curve agrees with and extends the bifurcation curves obtained in [6] by analyzing the equilibrium states of the ODE system. We showed that the instability of standing waves above the curve leads to the emergence of stable traveling waves in some parameter regimes, while at other parameter values traveling waves exhibit either frontal or background instability. An analysis of the background steady states provided information about the spectrum of these waves (Appendix A). From an applications perspective, a two-dimensional collection of oscillators, corresponding to cells in the retina or in a layer of visual cortex, is most relevant, and for this reason we also considered some prototypical examples of two-dimensional evolution. In particular, we demonstrated that perturbed planar fronts can heal and resume their planar form, while radial fronts may either shrink or flatten out into a square-like shape and expand, depending on the phase value inside and the parameter values. Our simulations of the system that initially has two fronts with bulging centers are in qualitative agreement with Carpenter's experimental observations [4] (see also the discussion of [5]). 

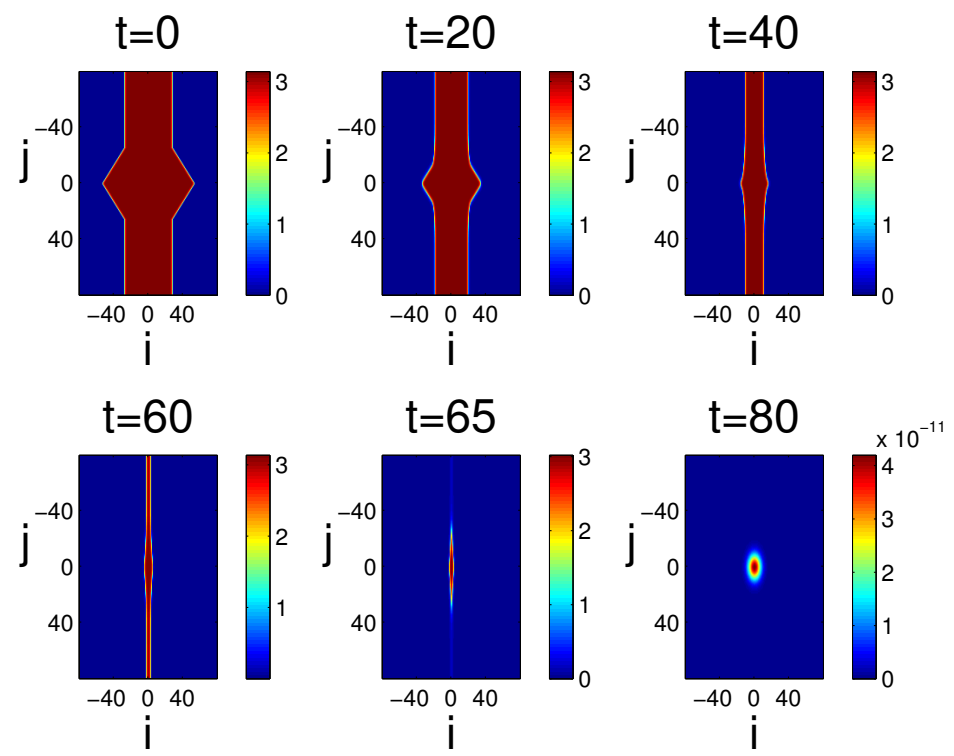

Figure 11: Snapshots of two outwardly perturbed fronts at times $t=0,20,40,60,65$ and 80 when $k=1.3, \mu=0.5$. The fronts meet near the domain edges, form a loop, and shrink.

The present work leads to numerous interesting questions for the further exploration of this and related systems. In particular, obtaining an analytical handle on the spectrum of the front in the co-traveling wave PDE and connecting this spectrum to the stability properties of the original ODEs would be extremely valuable from a theoretical perspective, not only in the context of the present setting but also for wide additional classes of lattice dynamical problems bearing traveling waves, such as generalized Frenkel-Kontorova (see [9] and references therein), Fermi-Pasta-Ulam (see e.g. [10, 11, 12, 13, 14, 15] and the discussion of Chapter 1 in [16]) or nonlinear Schrödinger systems (see e.g. [17, 18, 19, 20, 21, 22] and the discussion of Chapter 21 in [23]). On the numerical front, while we explored a few prototypical cases of twodimensional evolution, a better understanding of the stationary and traveling states in two dimensions clearly merits further investigation. Another interesting direction is to formulate and study solutions and spectra of a two-component problem that may support traveling pulses.

On the applications side, once the basic properties of this model system 

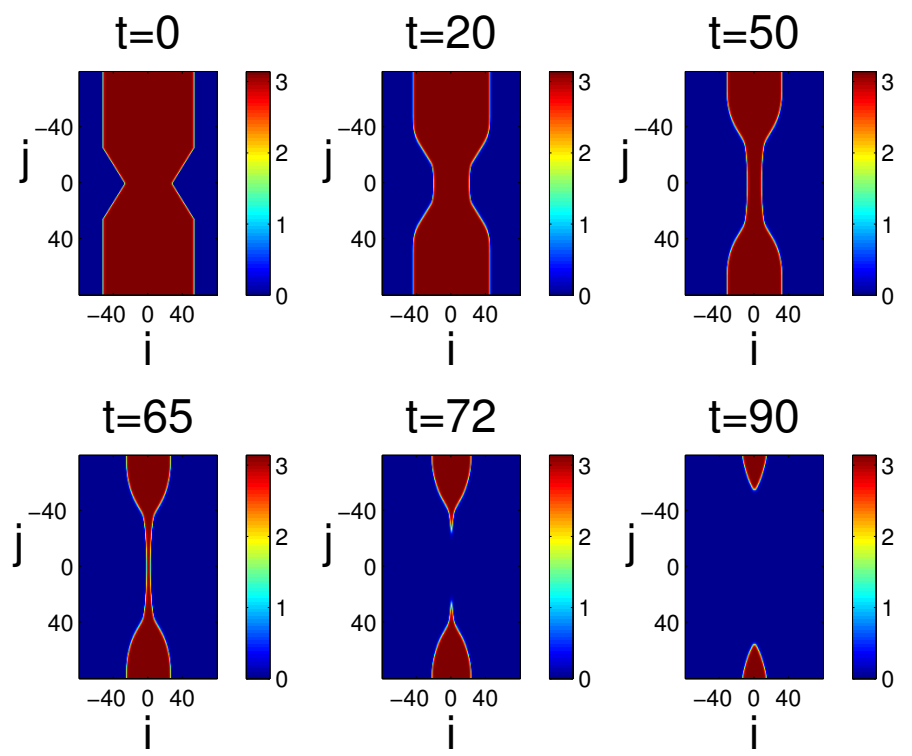

Figure 12: Snapshots of two inwardly perturbed fronts at times $t=0,20,50,65,72$ and 90 when $k=1.3, \mu=0.5$. The fronts meet near their centers, separate, and evolve into two (upper and lower) parts.

are more fully understood, it may complement other models (e.g., [5]) as a form of computational test bed for exploring and making predictions about patterns emerging from forcing of coupled oscillator networks, such as visual phenomena evoked by electrical stimulation that do not depend specifically on the complex biological details of the neurons involved. In this context, it may be interesting to explore the influence of changes in the amplitude or qualitative form of the forcing function on wave propagation [3] and to investigate waves induced by presenting traveling wave stimuli (e.g., [24, 25]), representing objects passing through the visual field, in addition to periodic forcing. Indeed, these adjustments may help resolve the model's current failure to capture the observation that neighboring fronts tend to propagate in a similar manner. At a more fundamental level, the present class of models appears to be a (modified) overdamped variant of the widely studied so-called sine lattices (see e.g. [26, 27] and, for a discussion of some of the relevant applications, [28]), hence it would be particularly interesting to consider models of this type that include both inertia and dissipation terms and connect the 

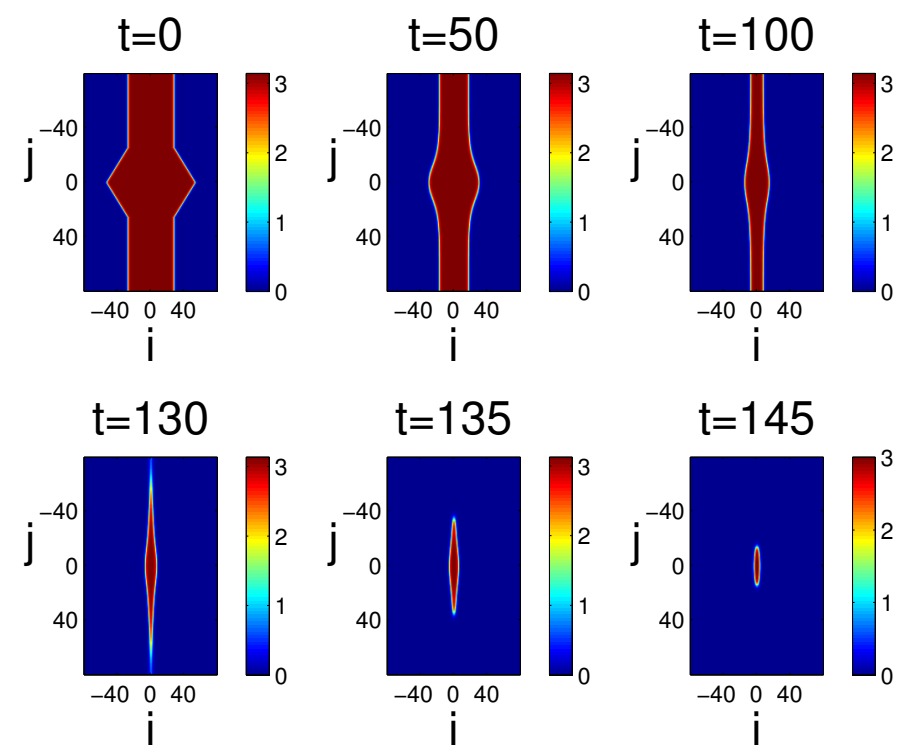

Figure 13: Snapshots of two outwardly perturbed fronts at times $t=0,50,100,130,135$ and 145 when $k=2, \mu=0.15$. As in Fig. 11, the fronts meet near the domain edges, form a loop, and shrink.

previously explored overdamped and undamped limits. Some of these issues are currently under investigation and will be reported upon in future publications.

Acknowledgments. PGK gratefully acknowledges the support of the USAFOSR under grant FA9550-12-1-0332, and the ERC under FP7, Marie Curie Actions, People, International Research Staff Exchange Scheme (IRSES605096). Some of PGK's work on the manuscript was done during his oneyear residence as an Ulam fellow at the Center for Nonlinear Studies, Los Alamos National Laboratory, supported in part by the U.S. Department of Energy. JER was partially supported by the NSF award DMS-1312508.

\section{Appendix A. Background instability}

We now discuss the stability of the background states with $\Theta_{0}=0$ or $\Theta_{0}=\pi$ and their relation to the stability of the traveling wave solutions that 

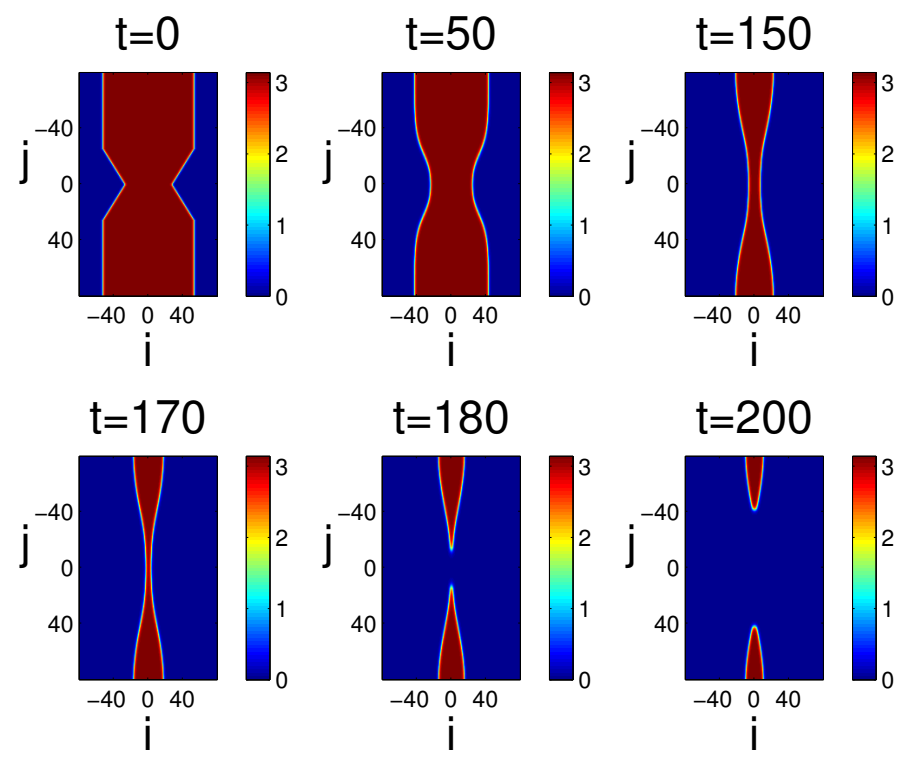

Figure 14: Snapshots of two inwardly perturbed fronts at times $t=0,50,150,170,180$ and 200 when $k=2, \mu=0.15$. As in Fig. 12, the fronts meet near their centers, separate, and evolve into two (upper and lower) parts.

we consider. Our traveling wave solutions clearly have support on both of these states. In fact, the nonlinear system resulting from the discretization of Eq. (5) contains very few equations at the front, while most of the equations involve the background. Therefore the spectrum of these homogeneous states should be mirrored within that of our (standing or traveling) waves. In other words, we are implicitly assuming here that the spectrum of a front solution contains two principal "ingredients", namely the point spectrum associated with the front itself, and the continuous spectrum associated with the background on which the front resides.

Substituting $\Theta(z, \tau)=\Theta_{0}+\epsilon v(z, \tau)$ in Eq. (3), where $\epsilon$ is a small parameter, and linearizing about $\Theta_{0}$, one obtains the following linear advance-delay partial differential equation with constant coefficients for $v(z, \tau)$ :

$$
v_{\tau}-c v_{z}=k \cos (\mu)(v(z+1, \tau)-2 v(z, \tau)+v(z-1, \tau))-2 v(z, \tau) .
$$

Seeking solutions of (A.1) in the form $v=e^{\lambda \tau} e^{i p z}$ for real $p$, we have

$$
\lambda=-2\left(2 k \cos (\mu) \sin ^{2}(p / 2)+1\right)+i c p .
$$


The equilibrium state $\Theta(z, \tau) \equiv \Theta_{0}$ is unstable when $\operatorname{Re} \lambda>0$, i.e. when

$$
k \cos (\mu)<-\frac{1}{2 \sin ^{2}(p / 2)}
$$

for some real $p$. Since $k>0$, this can happen for example at large enough $k$ and $\frac{\pi}{2}<\mu<\frac{3 \pi}{2}$. More precisely, the background solution is always unstable for

$$
k>\frac{1}{2}|\sec (\mu)|, \quad \cos (\mu)<0 .
$$

Additionally, since (A.2) implies that $\operatorname{Re} \lambda=-2\left(2 k \cos (\mu) \sin ^{2}(p / 2)+1\right)$ and $\operatorname{Im} \lambda=c p$, these eigenvalues determine the following locus of points in the spectral plane:

$$
\operatorname{Re} \lambda=-2\left\{2 k \cos (\mu) \sin ^{2}\left(\frac{\operatorname{Im} \lambda}{2 c}\right)+1\right\}
$$

which should be traceable in the spectra of the linearized operators for the traveling waves considered.

From Eq. (A.4), one can see that the background is always stable for $-\pi / 2+2 \pi j \leq \mu \leq \pi / 2+2 \pi j$, where $j$ is any integer, so any instability in this parameter regime must come from the front itself. For example, Fig. A.15(a) shows the eigenvalues of the Jacobian matrix associated with the traveling wave solution at $\mu=0.5$ and $k=1.5$. The eigenvalues are given for $n=2001,4001$ and 16001 nodes in our discretization of the co-traveling wave problem (3) on the interval [-200,200], with the step size $h=400 /(n-1)$. The eigenvalues of the linearized equation about the background solutions $\Theta_{0}=0$ and $\Theta_{0}=\pi$ are given by Eq. (A.2). For these parameter values, Eq. (A.4) yields $-7.27 \leq \operatorname{Re} \lambda \leq-2$. In Fig. A.15(a), one can see a band of eigenvalues folding towards the imaginary axis as $n$ increases. Zooming in on the region near the imaginary axis in Fig. A.15(b), we see that the eigenvalues are converging to the spectrum of the background state as the continuum limit is approached, as predicted.

In the Appendix B, we explain further how the forward difference scheme we use results in the particular structure of the continuous spectrum of the problem (inducing the shape observed in Fig. A.15(a)) and how a centered difference scheme would affect the corresponding spectral and stability picture. Similar results are found for the cases of $k=2.25$ and $k=1.1$ (not shown here). 


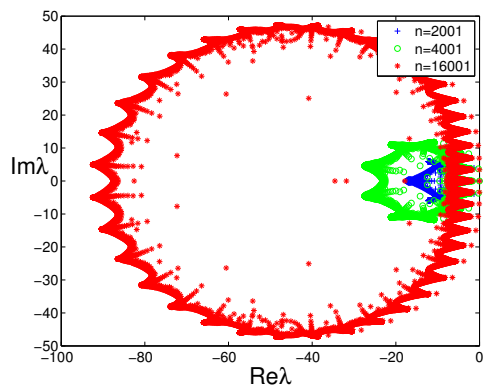

(a)

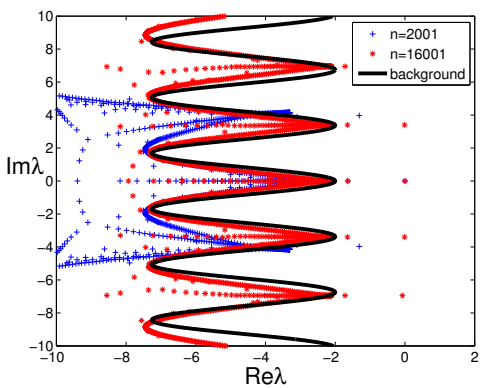

(b)

Figure A.15: (a) The eigenvalues of the Jacobian for the traveling wave solution at $\mu=0.5$, $k=1.5$ discretized on the interval $[-200,200]$ with $n=2001,4001$ and 16001 nodes. (b) The enlarged version of the $n=2001$ and $n=16001$ cases from (a), along with the curve ("background") given by Eq. (A.4) and showing the continuous spectrum of the background state, near the imaginary axis.

In Sec. 3 the analysis of traveling waves was limited, as in the study of the equilibrium states in [6], to the physically relevant interval $0<\mu<\pi / 2$. We now extend our analysis to the entire upper half of the $(\mu, k)$ plane. To this end, we first observe that Eq. (5) is $2 \pi$-periodic in $\mu$, implying that its solutions (both standing and traveling waves) are also $2 \pi$-periodic in $\mu$. The solutions are also symmetric about $\mu=\pi n$, where $n$ is any integer. Indeed, consider a traveling wave solution $\phi(z)$ at $(\mu, k)$ with velocity $c$. Defining new variables $\bar{c}=-c, \bar{\mu}=2 \pi n-\mu, \bar{z}=-z$ and setting $\bar{\phi}(\bar{z})=\pi-\phi(z)$, we obtain $\phi^{\prime}(z)=\bar{\phi}^{\prime}(\bar{z}), \phi(z+1)=\pi-\bar{\phi}(\bar{z}-1)$ and $\phi(z-1)=\pi-\bar{\phi}(\bar{z}+1)$. Substituting this in Eq. (5) and using the symmetry and periodicity of $H(\theta)$ and $f(\theta)$, we obtain, after simplification,

$$
\begin{aligned}
-\bar{c} \bar{\phi}^{\prime}(\bar{z}) & =k(\sin (\bar{\phi}(\bar{z}+1)-\bar{\phi}(\bar{z})+\bar{\mu})+\sin (\bar{\phi}(\bar{z}-1)-\bar{\phi}(\bar{z})+\bar{\mu})-2 \sin (\bar{\mu})) \\
& -\sin (2 \bar{\phi}(\bar{z})),
\end{aligned}
$$

which in fact coincides with Eq. (5) for our choice of $H(\theta)$ and $f(\theta)$. Hence, $\bar{\phi}(\bar{z})$ is a solution of Eq. (5) at $\bar{\mu}=2 \pi n-\mu$ with velocity $\bar{c}=-c$. Note that $\mu-\pi n=\pi n-\bar{\mu}$, so this is the symmetry about $\mu=\pi n$. Thus, for any traveling wave $\phi(z)$ at $(\mu, k)$, there is a traveling wave $\pi-\phi(-z)$ at $(2 \pi n-\mu, k)$ which has the same speed and spectrum as the wave with $(\mu, k)$ but propagates in the opposite direction. Similar analysis shows that for every solution $\phi(z)$ at $(\mu, k)$ there is another solution $\phi(-z)$, a reflection, 
that has the opposite velocity and the same spectrum. These results are summarized in Table A.1. The same symmetry and periodicity clearly also

Table A.1: Symmetry of the traveling wave solutions

\begin{tabular}{ccc}
\hline \hline$(\mu, k)$ & solution & velocity \\
\hline$(\mu, k)$ & $\phi(z)$ & $c$ \\
$(\mu, k)$ & $\phi(-z)$ & $-c$ \\
$(2 \pi n-\mu, k), n \in \mathbb{Z}$ & $\pi-\phi(z)$ & $c$ \\
$(2 \pi n-\mu, k), n \in \mathbb{Z}$ & $\pi-\phi(-z)$ & $-c$ \\
\hline
\end{tabular}

hold for the standing waves $(c=0)$, the equilibrium solutions $\theta_{j}$ of $(2)$.

By periodicity, it suffices to consider $-\pi<\mu \leq \pi$. Pitchfork and saddlenode bifurcation curves extended to this interval are shown in Fig. A.16 along with the curves above which the background is unstable, according to the inequality (A.3) obtained above for the linearized problem. The curve separating standing and traveling waves coincides with the saddle-node bifurcation curves where they exist and follows the pitchfork bifurcation curve otherwise. Using the $2 \pi$-periodicity, the curves can be extended to the entire upper half of the $(\mu, k)$ plane.

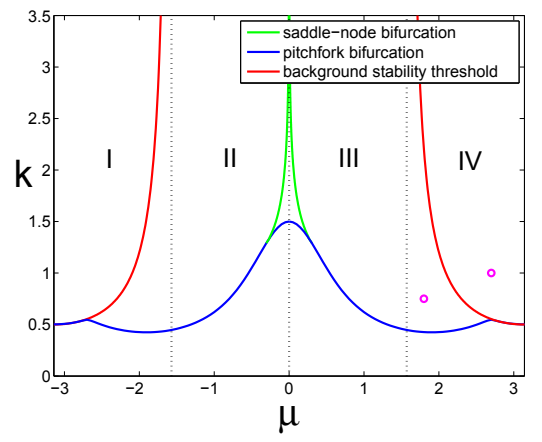

Figure A.16: Extended pitchfork (blue) and saddle-node (green) bifurcation curves and the curves (red) above which the background is unstable. Magenta circles denote the parameter values where the stability of the traveling waves was probed via direct numerical computations.

To explore the stability of traveling wave solutions where they exist, we divide the parameter space into several regions corresponding to different 
intervals of $\mu$. These are the $\mu$ regions $I:\left(-\pi,-\frac{\pi}{2}\right], I I:\left(-\frac{\pi}{2}, 0\right), I I I:\left(0, \frac{\pi}{2}\right)$, and $I V:\left[\frac{\pi}{2}, \pi\right]$. Traveling waves in the region III have already been studied in Sec. 3, with numerical simulations suggesting their stability. By the symmetry mentioned above, these results readily extend to region II.

It thus remains to consider region $I V$, since by symmetry we then also obtain results for region $I$, where the corresponding waves have the opposite velocity. With this in mind, we select $(\mu, k)$ in region $I V$ and solve the traveling wave equation (5) with the boundary conditions $\phi(z) \rightarrow \pi$ as $z \rightarrow$ $\infty, \phi(z) \rightarrow 0$ as $z \rightarrow-\infty$ for $\phi(z)$ and velocity $c$. Using the forward difference or in some cases the centered difference approximation yields convergence to the relevant solutions. As before, we then use the resulting traveling wave as initial data to solve the ODE system (1) in order to check its stability and visualize the resulting dynamics. To obtain the initial condition, we evaluate the traveling wave solution at 51 integer points, and then place it on a grid of 81 points by padding it with $0 \mathrm{~s}$ on the left and $\pi \mathrm{s}$ on the right:

$$
\theta_{j}(0)= \begin{cases}0, & j=-40, \ldots,-26 \\ \phi(j), & j=-25, \ldots, 25 \\ \pi, & j=26, \ldots, 40 .\end{cases}
$$

The padding introduces a small perturbation of the initial curve, which, when the solution is unstable, initiates the instability. The points $(\mu, k)=$ $(1.8,0.75)$ and $(2.7,1)$, (marked by circles in Fig. A.16) are chosen as representative points from the region $I V$.

We start by considering he point $(\mu, k)=(2.7,1)$, which lies in the region where the background state was shown to be unstable, above the red curve in Fig. A.16. In this case the traveling wave moves to the right with velocity $c=$ 0.2233. In panel (a) of Fig. A.17, the initial condition (perturbed traveling wave) is shown. In panel (b), the solution is shown at time $t=4$, when the perturbation of the unstable solution has caused the relevant dynamical instability to be manifested in the $\theta \approx 0$ part of the solution. In panel (c), the solution is shown at time $t=8.5$, when the instability of the background is manifested at both ends of the domain. Finally, panel (d) gives the spacetime contour plot up to time $t=50$, clearly illustrating the destabilization of the background and where it is initiated.

Fig. A.18(a) shows time snapshots of the solution of the ODE system (1) initialized by the traveling wave (solid blue curve) obtained from Eq. (5) at $(\mu, k)=(1.8,0.75)$, which lies in the region $I V$ below the background in- 


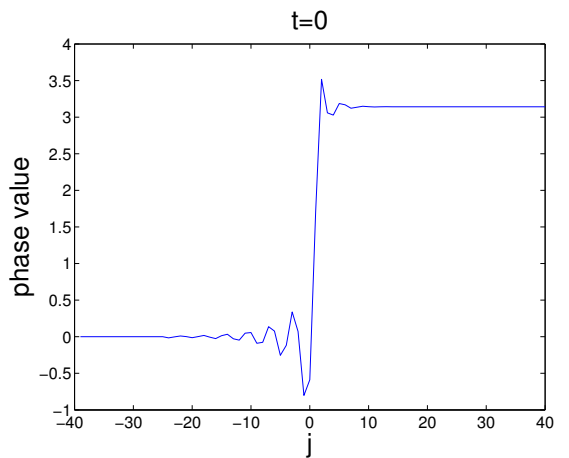

(a)

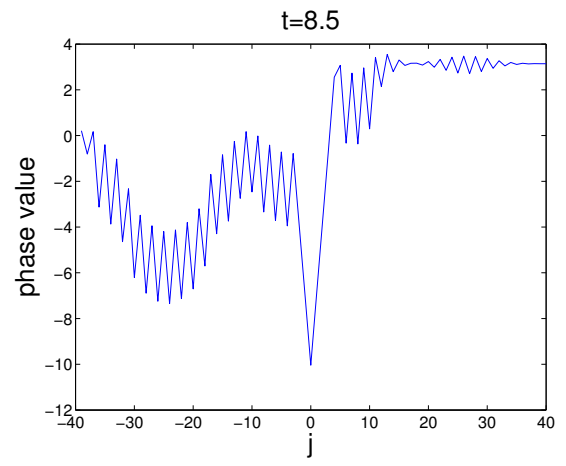

(c)

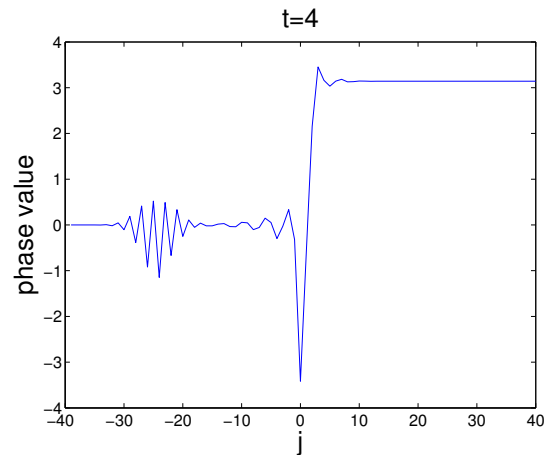

(b)

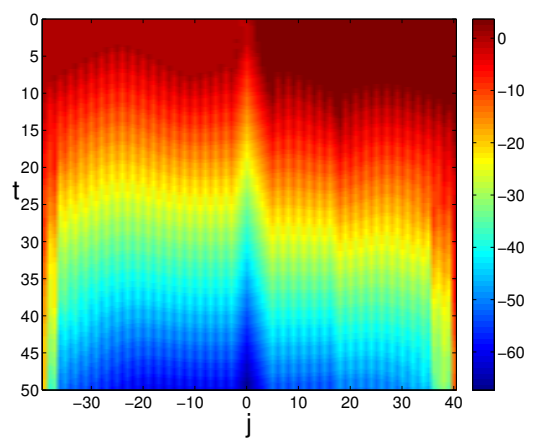

(d)

Figure A.17: Unstable traveling wave at $(\mu, k)=(2.7,1)$ with $c=0.2233$ : (a) Initial state $(t=0)$ obtained from the solution of Eq. (5). (b) Solution of Eq. (1) at $t=4$ where the left side of the solution, with $\theta_{j} \approx 0$, manifests destabilization. (c) Solution at $t=8.5$ where the right side, with $\theta_{j} \approx \pi$, also shows destabilization. (d) Contour plot of spacetime evolution until time $t=50$. Here the color code represents $\theta_{j}$ values on the real line, rather than in $[0,2 \pi) \bmod 2 \pi$.

stability curve. The space-time contour plot of the solution is shown in Fig. A.18(b). The traveling wave is initially traveling to the right at the positive predicted velocity $c=0.5493$. However, a frontal instability of the traveling wave leads to the formation of two fronts near $t=7.28$ that can be seen at $t=14.56$ (dashed-dotted green curve) and $t=21.85$ (dotted magenta curve) in Fig. A.18(a), as well as in the contour plot shown in Fig. A.18(b). Interestingly, the two fronts propagate in the opposite directions with the same speed as the initial unstable traveling wave. In contrast to the solution at $(2.7,1)$, which featured background instability, no instability of the back- 


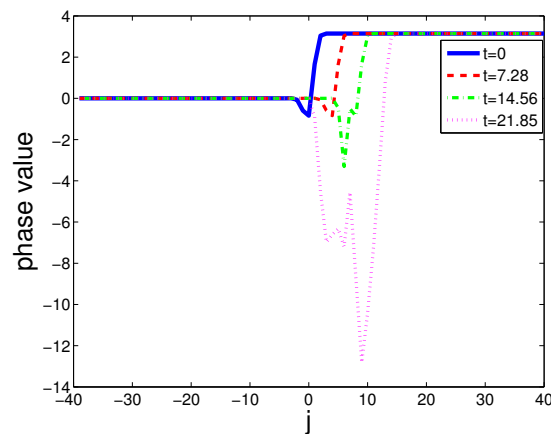

(a)

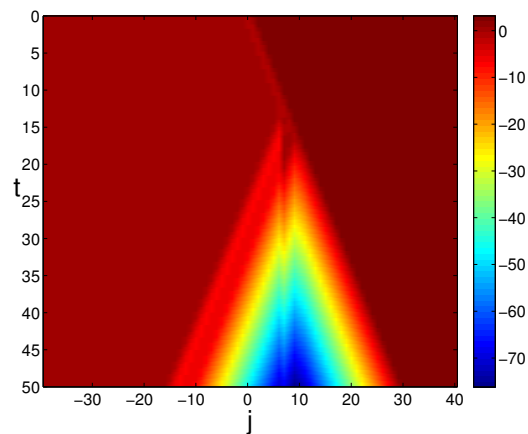

(b)

Figure A.18: Unstable traveling wave at $(\mu, k)=(1.8,0.75)$ with $c=0.5493$ : (a) Snapshots of the solution of Eq. (1) initialized at the traveling wave at times $t=0$ (solid blue curve), 7.28 (approximate onset of instability, dashed red), 14.56 (dash-dotted green) and 21.85 (dotted magenta). (b) The contour plot of space-time evolution of the solution (with $\theta \in \mathbb{R}$ ) until time $t=50$. The traveling wave becomes unstable after an initial transient propagation period and splits into two fronts propagating in the opposite directions with the same speed as the initial wave.

ground is observed in this example, and only the frontal instability arises. At $k=0.75$, the frontal instability exists at least for $1.65<\mu<q$, where $q$ satisfies $0.75=0.5|\sec (q)|$ based on equation (A.3); that is, until $\mu$ reaches the background instability region. No instability is observed for traveling waves at $\mu \leq 1.65$ and $k=0.75$ over the time interval $[0,500]$.

The results of our stability investigations are summarized in Table A.2. We emphasize that curves indicating the onset of background instability in the $(\mu, k)$-plane do not coincide with the vertical lines $\mu=-\pi / 2$ and $\mu=\pi / 2$ bounding regions $I$ and $I V$. In particular, our numerical results indicate that the curve located in region $I V$ asymptotes slightly to the right of the vertical line $\mu=\pi / 2$. By the symmetry described above there is a similar stability boundary in region I slightly to the left of the line $\mu=-\pi / 2$.

In summary, we have obtained an analytical expression for the boundary of a region in parameter space where traveling waves suffer a background instability. Numerical simulations show that as parameters are varied from the zero-speed boundary of the traveling wave region, traveling waves can be stable or can undergo either a background instability (within the region predicted analytically) or a frontal instability (between the stable region and the background instability region). Due to the periodicity of $H(\theta)$ and $f(\theta)$, 
Table A.2: Stability and velocities of traveling waves

\begin{tabular}{cccc}
\hline \hline$(\mu, k)$ & region & stability & velocity \\
\hline$(-2.7,1)$ & $I$ & unstable (background) & -0.2233 \\
$(-1.8,0.75)$ & $I$ & unstable (frontal) & -0.5493 \\
$(-1.63,1)$ & $I$ & stable & -0.8678 \\
$(-1,1)$ & $I I$ & stable & -0.6214 \\
$(-0.5,1.1)$ & $I I$ & stable & -0.2382 \\
$(-0.5,1.5)$ & $I I$ & stable & -0.5368 \\
$(-0.5,2.25)$ & $I I$ & stable & -0.8123 \\
$(0.5,2.25)$ & $I I I$ & stable & 0.8123 \\
$(0.5,1.5)$ & $I I I$ & stable & 0.5368 \\
$(0.5,1.1)$ & $I I I$ & stable & 0.2382 \\
$(1,1)$ & $I I I$ & stable & 0.6214 \\
$(1.63,1)$ & $I V$ & stable & 0.8678 \\
$(1.8,0.75)$ & $I V$ & unstable (frontal) & 0.5493 \\
$(2.7,1)$ & $I V$ & unstable (background) & 0.2233 \\
\hline
\end{tabular}

all results repeat periodically in $\mu$ with the period of $2 \pi$.

\section{Appendix B. Some details on numerical methods}

To solve the traveling wave Eq. (5), we used a second order forward difference scheme and sometimes a second order centered difference scheme to approximate the term $\phi^{\prime}(z)$. For a grid point $j$, these approximations are of the form $\left(-3 \theta_{j}+4 \theta_{j+1}-\theta_{j+2}\right) /(2 \Delta x)$ and $\left(\theta_{j+1}-\theta_{j-1}\right) /(2 \Delta x)$, respectively, where $\Delta x$ is the grid spacing. In some cases, the forward difference approximation used in solving Eq. (5) did not converge but the centered difference approximation did. Whichever approximation was used to solve Eq. (5) for the traveling wave $\phi(z)$, it was checked that the solution of the ODE system (1) with the initial condition $\theta_{i}(0)=\phi(i)$ produced results that were consistent with the obtained traveling wave solution.

In the following we analyze the stability of the background state in the discretized Eq. (5) with the forward and centered difference approximations described above. First, the forward difference approximation is analyzed. In Fig. A.15, we see that the eigenvalues of the Jacobian for Eq. (5) approximate the eigenvalues of the continuum background as the number of points is increased, although the full structure of the forward difference spectral locus 
is more complex. The eigenvalues for the background can also be obtained in the context of the forward difference as follows. In this case, Eq. (A.1) obtained by linearizing Eq. (3) about the background equilibrium state $\Theta_{0}=$ 0 or $\Theta_{0}=\pi$ is replaced by

$$
\begin{aligned}
& V_{\tau}-c\left[\frac{-V(J+2, \tau)+4 V(J+1, \tau)-3 V(J, \tau)}{2 \Delta x}\right]= \\
& k \cos (\mu)[(V(J+q, \tau)-2 V(J, \tau)+V(J-q, \tau))]-2 V(J, \tau),
\end{aligned}
$$

where $V(J, \tau)$ is the approximation of $v(z, \tau)$ at a grid point $z_{J}=J \Delta x$ for integer $J$ and $q$ is an integer such that $q \Delta x=1$. Seeking solutions in the form $V_{J}(\tau)=e^{\lambda \tau} e^{i J p \Delta x}$, where $p$ is the wave number, and solving for $\lambda$, we find that

$$
\begin{aligned}
\lambda & =c\left[\frac{-\cos (2 p \Delta x)+4 \cos (p \Delta x)-3}{2 \Delta x}\right]+2 k \cos \mu(\cos (p)-1)-2 \\
& +i c\left[\frac{-\sin (2 p \Delta x)+4 \sin (p \Delta x)}{2 \Delta x}\right]
\end{aligned}
$$

The real and imaginary parts of these eigenvalues parametrized by $p$ are shown by red circles in Fig. B.19 at $k=2.25,1.5$, and 1.1 and $\mu=0.5$. For comparison, the eigenvalues of the Jacobian associated with the traveling wave solution are shown by blue pluses (recall also Fig. A.15, where these eigenvalues are shown for the case $k=1.5$ and $\mu=0.5$ for different numbers of nodes in the discretization). The plots show that the two sets of eigenvalues are quite close to each other.

Expanding Eq. (B.2) in a Taylor series at small $\Delta x$, we obtain

$\lambda=-\frac{c p^{4}(\Delta x)^{3}}{4}+2\{k \cos (\mu)(\cos (p)-1)-1\}+i\left(c p+c \frac{p^{3}}{3}(\Delta x)^{2}+O\left((\Delta x)^{4}\right)\right)$,

which yields Eq. (A.2) in the limit $\Delta x \rightarrow 0$. The principal part of the error in the real and imaginary parts is $-c \frac{p^{4}}{4}(\Delta x)^{3}$ and $c \frac{p^{3}}{3}(\Delta x)^{2}$, respectively. As the wave number $p$ increases, this error pushes the real part of $\lambda$ to $-\infty$ and the imaginary part to $+\infty$ and $-\infty$, again in line with the observations of Fig. A.15.

It is interesting to explore the spectral properties of the background of the traveling wave using a centered difference approximation instead of the 


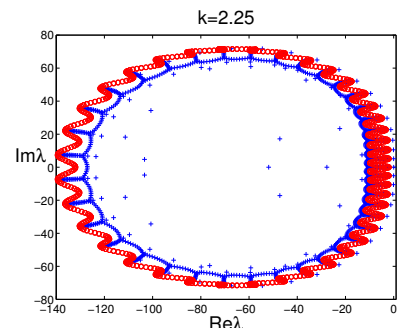

(a)

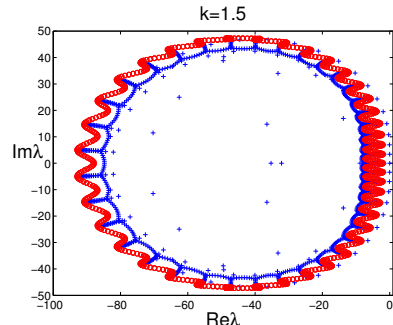

(b)

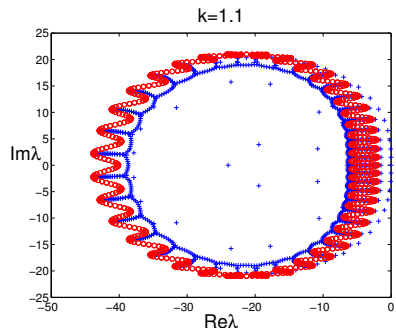

(c)

Figure B.19: Plot of the eigenvalues of the Jacobian associated with the linearization of Eq. (3) about the traveling wave solution (blue pluses), solved on [-25, 25] using 2001 nodes and a forward difference scheme, and the eigenvalues for the background equilibrium state (red circles) given by Eq. (B.2). Here $\mu=0.5$ and $k=2.25,1.5$, and 1.1.

forward difference approximation. Eq. (B.1) is then replaced by

$$
\begin{aligned}
V_{\tau}-c \frac{V(J+1, \tau)-V(J-1, \tau)}{2 \Delta x} & =k \cos (\mu)(V(J+q, \tau) \\
& -2 V(J, \tau)+V(J-q, \tau))-2 V(J, \tau),
\end{aligned}
$$

and the eigenvalues $\lambda$ are given by

$$
\lambda=2 k \cos (\mu)(\cos (p)-1)-2+i \frac{c \sin (p \Delta x)}{\Delta x} .
$$

In this case there is no error in the real part of $\lambda$.

Generally, this centered difference approximation is numerically unstable for the advection equation given by (3); see [29]. Here, we find that the nonlinear term does stabilize it for large enough values of $k$, but the instability in the numerical method is observed for smaller values of $k$ even though the solutions of the traveling wave equation are stable, according to the forward difference approximation. For example, in Fig. B.20 the eigenvalues obtained using the centered difference approximation of Eq. (5) are presented for $k=1.1,1.5$ and 2.25 with $\mu=0.5$. The real parts of the eigenvalues mostly lie between -5.86 and -2 for $k=1.1$, between -7.27 and -2 for $k=1.5$ and between -9.90 and -2 for $k=2.25$, which agree almost exactly with the continuum background theory based on Eq. (A.2). According to Fig. B.20, however, with fixed $\mu=0.5$, the centered difference approximation predicts that traveling waves become unstable somewhere between $k=1.5$ 


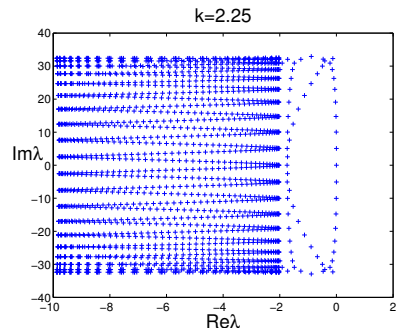

(a)

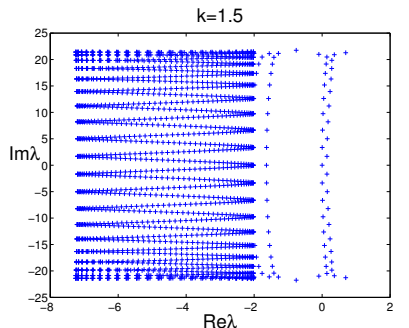

(b)

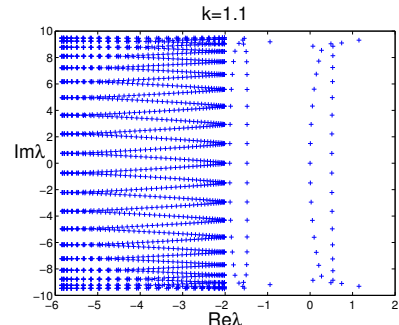

(c)

Figure B.20: Plot of the eigenvalues of the Jacobian associated with the linearization of Eq. (3) about the traveling wave solution (blue pluses), solved on [-25, 25] using 2001 nodes and centered difference approximation. Here $\mu=0.5$ and $k=2.25,1.5$ and 1.1.

and $k=2.25$, as some eigenvalues emerge with positive real part due to the instabilities associated with the centered difference approximation. In the results presented in the manuscript, care has been taken to avoid such spurious instabilities induced by the numerical scheme. 


\section{References}

[1] L. Cervetto, G. C. Demontis, C. Gargini, Cellular mechanisms underlying the pharmacological induction of phosphenes, British Journal of pharmacology 150 (4) (2007) 383-390.

[2] E. Zrenner, Will retinal implants restore vision?, Science 295 (5557) (2002) 1022-1025.

[3] E. J. Tehovnik, W. M. Slocum, Electrical induction of vision, Neuroscience \& Biobehavioral Reviews 37 (5) (2013) 803-818.

[4] R. H. S. Carpenter, Contour-like phosphenes from electrical stimulation of the human eye: some new observations, The Journal of Physiology 229 (3) (1973) 767-785.

[5] J. D. Drover, G. B. Ermentrout, Phase boundaries as electrically induced phosphenes, SIAM Journal on Applied Dynamical Systems 5 (4) (2006) $529-551$.

[6] H. F. Parks, G. B. Ermentrout, J. E. Rubin, The dynamics of a forced coupled network of active elements, Physica D 240 (7) (2011) 554-567.

[7] G. B. Ermentrout, Simulating, analyzing, and animating dynamical systems: a guide to XPPAUT for researchers and students, Vol. 14, SIAM, 2002.

[8] A. Carpio, L. L. Bonilla, Wave front depinning transition in discrete onedimensional reaction-diffusion systems, Physical Review Letters 86 (26) (2001) 6034.

[9] O. M. Braun, Y. S. Kivshar, The Frenkel-Kontorova model: concepts, methods, and applications, Springer, Berlin-Heidelberg, 2004.

[10] G. Friesecke, R. L. Pego, Solitary waves on FPU lattices: I. Qualitative properties, renormalization and continuum limit, Nonlinearity 12 (6) (1999) 1601-1626.

[11] G. Friesecke, R. L. Pego, Solitary waves on FPU lattices: II. Linear implies nonlinear stability, Nonlinearity 15 (4) (2002) 1343-1359. 
[12] G. Friesecke, R. L. Pego, Solitary waves on Fermi-Pasta-Ulam lattices: III. Howland-type Floquet theory, Nonlinearity 17 (1) (2004) 202-207.

[13] G. Friesecke, R. L. Pego, Solitary waves on Fermi-Pasta-Ulam lattices: IV. Proof of stability at low energy, Nonlinearity 17 (1) (2004) 229-251.

[14] J. English, R. L. Pego, On the solitary wave pulse in a chain of beads, Proceedings of the American Mathematical Society 133 (6) (2005) 17631768.

[15] A. Stefanov, P. Kevrekidis, Traveling waves for monomer chains with precompression, Nonlinearity 26 (2) (2013) 539-564.

[16] V. Nesterenko, Dynamics of heterogeneous materials, Springer-Verlag, New York, 2001.

[17] D. E. Pelinovsky, V. M. Rothos, Bifurcations of travelling wave solutions in the discrete NLS equations, Physica D 202 (1) (2005) 16-36.

[18] D. E. Pelinovsky, Translationally invariant nonlinear Schrödinger lattices, Nonlinearity 19 (11) (2006) 2695-2716.

[19] O. F. Oxtoby, I. V. Barashenkov, Moving solitons in the discrete nonlinear Schrödinger equation, Physical Review E 76 (3) (2007) 036603.

[20] T. R. O. Melvin, A. R. Champneys, P. G. Kevrekidis, J. Cuevas, Radiationless traveling waves in saturable nonlinear Schrödinger lattices, Physical Review Letters 97 (12) (2006) 124101.

[21] T. R. O. Melvin, A. R. Champneys, P. G. Kevrekidis, J. Cuevas, Travelling solitary waves in the discrete Schrödinger equation with saturable nonlinearity: Existence, stability and dynamics, Physica D 237 (4) (2008) 551-567.

[22] M. Syafwan, H. Susanto, S. M. Cox, B. A. Malomed, Variational approximations for traveling solitons in a discrete nonlinear Schrödinger equation, Journal of Physics A: Mathematical and Theoretical 45 (7) (2012) 075207.

[23] P. G. Kevrekidis, The discrete nonlinear Schrödinger equation: mathematical analysis, numerical computations and physical perspectives, Vol. 232, Springer-Verlag, Heidelberg, 2009. 
[24] S. E. Folias, P. C. Bressloff, Stimulus-locked traveling waves and breathers in an excitatory neural network, SIAM Journal on Applied Mathematics 65 (6) (2005) 2067-2092.

[25] G. B. Ermentrout, J. Z. Jalics, J. E. Rubin, Stimulus-driven traveling solutions in continuum neuronal models with a general smooth firing rate function, SIAM Journal on Applied Mathematics 70 (8) (2010) 3039-3064.

[26] S. Homma, S. Takeno, A coupled base-rotator model for structure and dynamics of DNA local fluctuations in helical twist angles and topological solitons, Progress of Theoretical Physics 72 (4) (1984) 679-693.

[27] S. Takeno, S. Homma, Kinks and breathers associated with collective sugar puckering in DNA, Progress of Theoretical Physics 77 (3) (1987) $548-562$.

[28] F. Zhang, Kink shape modes and resonant dynamics in sine-lattices, Physica D: Nonlinear Phenomena 110 (1) (1997) 51-61.

[29] K. W. Morton, D. F. Mayers, Numerical solution of partial differential equations, Cambridge University Press, 1994. 


\title{
Traveling Wave Solutions in a Chain of Periodically Forced Coupled Nonlinear Oscillators
}

\author{
M. Duanmu, ${ }^{1}$ N. Whitaker, ${ }^{2}$ P. G. Kevrekidis, ${ }^{1,3}$ A. Vainchtein,${ }^{4}$ and J. Rubin ${ }^{5}$ \\ ${ }^{1}$ Department of Mathematics and Statistics, \\ University of Massachusetts, Amherst MA 01003-4515, USA \\ ${ }^{2}$ Department of Mathematics and Statistics, University of Massachusetts, \\ Amherst MA 01003-4515, USA, Telephone:413-545-1572, \\ FAX:413-545-1801, Email: whitaker@math.umass.edu \\ ${ }^{3}$ Center for Nonlinear Studies and Theoretical Division, \\ Los Alamos National Laboratory, Los Alamos, New Mexico 87545, USA \\ ${ }^{4}$ Department of Mathematics, University of Pittsburgh, Pittsburgh, PA 15260, USA \\ ${ }^{5}$ Department of Mathematics and Center for the Neural Basis of Cognition, \\ University of Pittsburgh, Pittsburgh, PA 15260, USA
}

\begin{abstract}
Motivated by earlier studies of artificial perceptions of light called phosphenes, we analyze traveling wave solutions in a chain of periodically forced coupled nonlinear oscillators modeling this phenomenon. We examine the discrete model problem in its co-traveling frame and systematically obtain the corresponding traveling waves in one spatial dimension. Direct numerical simulations as well as linear stability analysis are employed to reveal the parameter regions where the traveling waves are stable, and these waves are, in turn, connected to the standing waves analyzed in earlier work. We also consider a two-dimensional extension of the model and demonstrate the robust evolution and stability of planar fronts and annihilation of radial ones. Finally, we show that solutions that initially feature two symmetric fronts with bulged centers evolve in qualitative agreement with experimental observations of phosphenes.
\end{abstract}

Keywords: coupled nonlinear oscillators, discrete model, traveling waves

\section{INTRODUCTION}

Electrical stimulation of the retina can produce artificial perceptions of luminance changes called phosphenes, which may also arise in early stages of retinal or visual disease [1]. The induction of phosphenes is being used to help restore vision or develop visual aids for patients with severely compromised vision $[2,3]$, and an understanding of how phosphenes arise and behave could contribute to such efforts. In a detailed experimental study [4], Carpenter explored electrically induced 
phosphenes in human subjects. Each subject's eyes were immersed in a saline bath to which an alternating current was applied. When a dark object was passed through a subject's visual field in the presence of such stimulation, visual perceptions of line phosphenes occurred in its wake. The lines were observed to move and interact but never cross. This work suggested the presence of a bistability of activity states in the system, with the moving lines representing boundaries between sets of cells in different activity regimes.

Drover and Ermentrout [5] developed a one-dimensional model providing a simple representation of the phosphenes in Carpenter's experiments and their motion. In their work, a chain of excitable neurons was driven by a spatially uniform periodic stimulus at a frequency higher than the cells could follow, inducing a 1:2 phase locking with the stimulus. In the absence of coupling, neurons could fire on even or odd cycles of the stimulus, resulting in an intrinsic bistability for the forced system. Sufficiently strong coupling, even if directionally unbiased, elicited unidirectional traveling waves in which cells were recruited to switch phase. Large amplitude forcing of an excitable system is a difficult problem to tackle analytically. In view of that difficulty, the more recent analysis of [6] assumed that each neuron is intrinsically oscillatory and characterized by a state evolving at half the frequency of an applied forcing signal. Using multiple time scale expansion and the Fredholm alternative, the authors of [6] derived a more analytically tractable effective model for the time evolution of the neurons' phases. This reduced model, which is quite general and particularly interesting in its own right, will be the focus of the present work.

In [6], a detailed numerical existence and stability analysis was done using XPPAUT [7] for a finite chain of coupled phase oscillators. Certain interesting bifurcation phenomena were identified including saddle-node and pitchfork bifurcations in a two-dimensional parameter space characterizing the strength and asymmetry of coupling between nearest-neighbor oscillators. Beyond the critical points involved in these bifurcations, direct numerical simulations identified traveling waves that are strongly reminiscent of the "recruitment waves" obtained in the original chain of forced neural oscillators. It is exactly these traveling waves that we systematically obtain and analyze in the present manuscript, by a combination of numerical and, whenever possible, semi-analytical techniques.

Specifically, we consider the exact traveling wave problem, which takes the form of an advancedelay differential ordinary equation in the co-traveling frame of such waves and which we introduce along with the mathematical formulation of the problem in Section 2. In Section 3, we proceed to solve this problem numerically, identifying exact (up to numerical error) traveling and standing (zero-speed) waves within the full two-dimensional parameter space used in [6]. We explore the 
stability of these waves in two complementary ways. On the one hand, we consider the traveling waves as steady states of the associated advance-delay partial differential equation (PDE). On the other hand, we examine them via direct numerical simulations of the original system of ordinary differential equations (ODEs) for the coupled oscillators with the initial condition "distilled" on the lattice from the obtained traveling wave solution. In [6], this problem was studied in a limited range for the parameter $\mu$ measuring the asymmetry of the nearest-neighbor coupling function. Here, our analysis extends to all values of $\mu$. We show that traveling waves are stable in certain parameter regions that are periodic in $\mu$ and are located above a certain curve, below which there exist stable standing waves. The stability regions alternate along the direction of $\mu$ with regions where the waves are unstable due to the instability of the background state as well as a frontal instability. Simulations of the lattice system initialized by an unstable traveling wave show that the frontal instability results in formation of two fronts that propagate in the opposite directions with the same speed as the initial wave.

In the final part of Section 3, we extend the lattice model to a two-dimensional setting and show that the planar fronts obtained from the one-dimensional traveling wave are very robust even with a local initial distortion, unlike radial fronts, which are eventually annihilated in the dynamical evolution. Finally, we consider the evolution of two symmetric fronts with initially bulged centers and show that the resulting dynamics is in agreement with Carpenter's findings, based on observations of phosphenes, that lines form loops instead of crossing through each other and that a line does not break apart unless it meets another line. This work adds support to the idea that a phase model may provide a useful framework for studying phosphenes and also raises interesting mathematical issues about the relation between advance-delay PDE and lattice ODE system solutions, and we conclude with a brief discussion of these points in Section 4 .

\section{DISCRETE MODEL AND THE TRAVELING WAVE EQUATION}

Consider a chain of $N$ periodically forced oscillators governed by the reduced spatially discrete model of stimulated retinal cells derived in $[6]$ :

$$
\begin{aligned}
\dot{\theta}_{-n} & =k H\left(\theta_{-n+1}-\theta_{-n}\right)+f\left(\theta_{-n}\right) \\
\dot{\theta}_{j} & =k\left[H\left(\theta_{j-1}-\theta_{j}\right)+H\left(\theta_{j+1}-\theta_{j}\right)\right]+f\left(\theta_{j}\right), \text { for } j=-n+1, \ldots, N-n-2 \\
\dot{\theta}_{N-n-1} & =k H\left(\theta_{N-n-2}-\theta_{N-n-1}\right)+f\left(\theta_{N-n-1}\right) .
\end{aligned}
$$

Here $\theta_{j}(t)$ is the slowly evolving phase of each neuron, $f(\theta)$ is a $\pi$-periodic forcing or locking function, and $H(\theta)$ is a $2 \pi$-periodic function characterizing the coupling of the nearest neighbors 
and multiplied by the coupling constant $k>0$. The periodicities of $f(\theta)$ and $H(\theta)$ represent the $1: 2$ frequency locking present in the system and can be easily generalized to different types of frequency locking. In [6] the number of oscillators was set to be even, $N=2 n$, but here we also allow it to be odd, $N=2 n+1$.

In what follows, we consider prototypical examples of the two functions proposed in [6],

$$
H(\theta)=\sin (\theta+\mu)-\sin (\mu), \quad f(\theta)=-\sin (2 \theta),
$$

where the parameter $\mu$ measures the asymmetry of the coupling function $H(\theta)$. We can identify 0 with $2 \pi$ such that $\theta_{j} \in[0,2 \pi)$ and then the firing of the $j$ th neuron corresponds to $\theta_{j}$ crossing through some distinguished value, taken for some oscillator models, for example, to be $\theta_{j}=\pi$. It is not hard to see that in the case of $\mu=0$, when $H(\theta)=\sin \theta$ is odd, there exist equilibrium split state (antiphase) solutions given by

$$
\left(\theta_{-n}, \ldots, \theta_{-1}, \theta_{0}, \ldots, \theta_{N-n-1}\right)=(0, \ldots, 0, \pi, \ldots, \pi) \text { and }(\pi, \ldots, \pi, 0, \ldots, 0)
$$

If $N=2 n$, these solutions have equal numbers of oscillators firing in each cycle. When $\mu$ is nonzero, such piecewise constant split states no longer exist. However, there are single-front equilibrium split states that are close to the above antiphase state but have boundary layers near the front. In [6], such steady state solutions of (1) are obtained numerically by varying $\mu$ and $k$. A prototypical example of the resulting diagram in the $(\mu, k)$ plane is shown in Fig. 1, adapted from Fig. 4 in [6]. There we observe that the primary split state solution is stable in a region of small enough $k$ (region A) but becomes unstable through a pitchfork bifurcation at $k=k^{*}(\mu)$ shown by the solid curve. This bifurcation is subcritical for $\mu$ above a certain threshold, $\mu>\mu_{c}$. For $\mu<\mu_{c}$, the bifurcation is supercritical and gives rise to two other, secondary, stable nonsymmetric split states that exist in region B. These states, in turn, disappear through a saddle-node bifurcation at $k=k^{* *}(\mu)$ (dashed curve), where $k^{* *}(\mu)>k^{*}(\mu)$. When exploring the dynamical byproducts of these instabilities, the authors of [6] identified a spontaneous emergence of traveling waves in region $\mathrm{C}$. This motivates us to seek (numerically) exact traveling wave solutions for this problem.

To this end, we consider the infinite chain of oscillators

$$
\dot{\theta}_{j}=k\left[H\left(\theta_{j-1}-\theta_{j}\right)+H\left(\theta_{j+1}-\theta_{j}\right)\right]+f\left(\theta_{j}\right), \quad j \in \mathbb{Z} .
$$

We start by seeking a solution of this system in the form $\theta_{j}(t)=\Theta(z, \tau)$, where $z=j-c t$ is a traveling wave coordinate with wave velocity $c$ and $\tau=t$, such that $\Theta \rightarrow 0$ and $\Theta \rightarrow \pi$ when $z \rightarrow-\infty$ and $\infty$, respectively, or vice versa. Substitution of this ansatz into (2) leads to the partial 


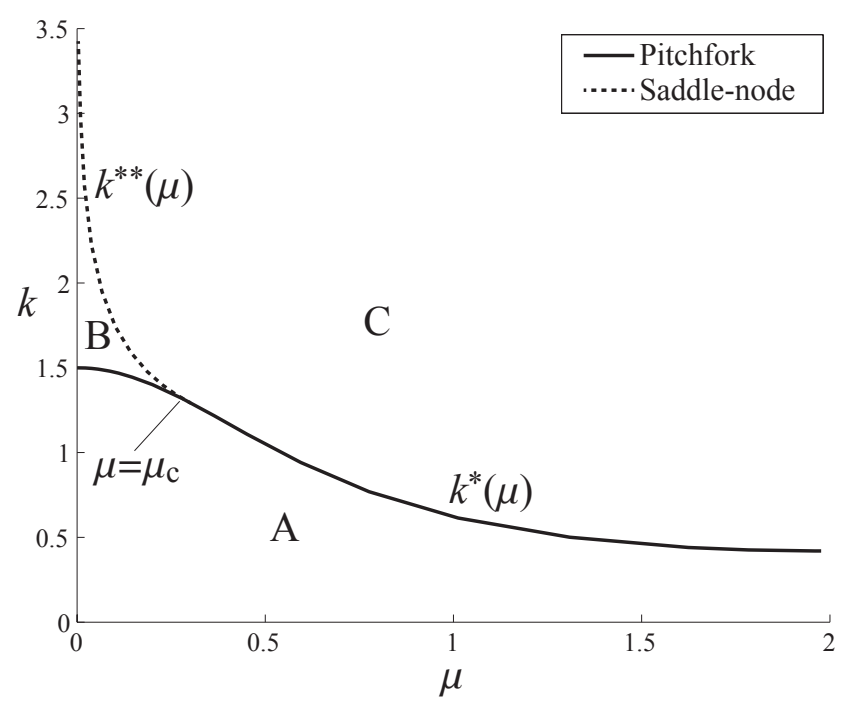

FIG. 1: Bifurcation diagram for stationary solutions of the system (1) with $N=2 n=10$, as obtained in [6]. The primary split state equilibria are stable in region A and destabilize via a pitchfork bifurcation (solid curve, $\left.k=k^{*}(\mu)\right)$. At $\mu<\mu_{c}$ the bifurcation is supercritical and gives rise to a pair of secondary split state equilibria that are stable in region B and disappear through a saddle-node bifurcation (dashed curve, $\left.k=k^{* *}(\mu), \mu<\mu_{c}\right)$.

differential advance-delay equation (co-traveling frame PDE) of the form

$$
\Theta_{\tau}-c \Theta_{z}=k[H(\Theta(z+1, \tau)-\Theta(z, \tau))+H(\Theta(z-1, \tau)-\Theta(z, \tau))]+f(\Theta(z, \tau)) .
$$

Traveling wave solutions of (2),

$$
\theta_{j}(t)=\phi(z), \quad z=j-c t
$$

are stationary solutions of (3) and satisfy the advance-delay ordinary differential equation

$$
-c \phi^{\prime}(z)=k[H(\phi(z+1)-\phi(z))+H(\phi(z-1)-\phi(z))]+f(\phi(z)) .
$$

Since every translate of the traveling solution is also a solution, the additional pinning condition $\phi(0)=\pi / 2$ is imposed on the nonlinear system in order to fix (i.e., pin) the traveling wave. This condition uniquely identifies the solution, as well as the corresponding velocity $c$. Note that the traveling wave ansatz (4) implies that $\theta_{j}(t)=\theta_{j+1}(t+1 / c)$ for any integer $j$, meaning that the phase value $\theta_{j}(t)$ is periodic (modulo shifts) with a period of $1 / c$. 


\section{EXISTENCE, STABILITY AND DYNAMICS}

\section{A. Examples of traveling wave solutions}

We start by considering solutions of the traveling wave Eq. (5) satisfying $\phi(0)=\pi / 2, \phi(z) \rightarrow \pi$ as $z \rightarrow \infty, \phi(z) \rightarrow 0$ as $z \rightarrow-\infty$. Eq. (5) is discretized in the interval $[-25,25]$ with a forward difference approximation of the derivative in the left hand side (for comparison purposes, a centered difference scheme was also used). The middle grid point is fixed to satisfy the pinning condition $\phi(0)=\pi / 2$, and the speed $c$ is added as a variable. We solve the resulting system using Newton's method for the traveling wave $\phi(z)$ and its velocity $c$, thus identifying numerically exact (at least up to a prescribed tolerance of $10^{-12}$ in the discretized system) solutions.

Examples of traveling wave solutions are shown in Figs. 2a-4a corresponding to different values of the parameters $k$ and $\mu$, characterizing the intersite coupling. In each case, stability of the traveling wave solution $\phi(z)$ is analyzed by computing the spectrum of the Jacobian matrix obtained by linearizing Eq. (3) about $\phi(z)$ with a forward difference approximation of the spatial derivative (see Appendix for further details); the resulting spectra are shown in Figs. 2b-4b. We also check stability of the obtained solutions by solving both the ODE system (1) and the advance-delay PDE (3) initialized at the traveling wave. Figs. 2c-4c show space-time evolution of the solution of the advance-delay PDE (3) with the initial condition $\Theta(z, 0)=\phi(z)$. The solution is obtained with the discretization grid used to obtain the traveling wave $\phi(z)$. As expected, the results show that $\phi(z)$ is a stationary solution of Eq. (3). Finally, in Figs. $2 d-4 d$, we show the space-time evolution of the solution of the ODE system (1) with $N=2 n+1=51$ oscillators initialized by the traveling wave solution $\phi(z)$ evaluated at the integer values of $z, \theta_{j}(0)=\phi(j), j=-25, \ldots, 25$. The time evolution of the PDE (3) and the ODE system (1) is accomplished using the classical fourth order Runge-Kutta method.

In Fig. 2a, the traveling wave solution $\phi(z)$ (solid line) is shown at $k=2.25$ and $\mu=0.5$, which yields $c=0.8123$. Fig. $2 \mathrm{~b}$ indicates that the eigenvalues of the linearization Jacobian of the PDE (3), evaluated at this solution, have only negative real parts, implying the linear (spectral) stability of the traveling wave solution as a stationary solution of Eq. (3). To further examine this prediction, the traveling wave solution is subsequently used as the initial data in Eq. (3), $\Theta(z, 0)=\phi(z)$, and the PDE is solved numerically, with the space-time contour of the evolution of $\Theta(z, \tau)$ shown in Fig. 2c confirming the robust evolution of this steady state. Finally, Fig. 2d shows the evolution of the solution of the discrete ODE system (1) initialized by the traveling wave 
solution, $\theta_{j}(0)=\phi(j)$. As anticipated by the traveling wave ansatz, the ODE solution is found to traverse the lattice at the velocity $c=0.8124$, which agrees with the traveling wave velocity very well [23].

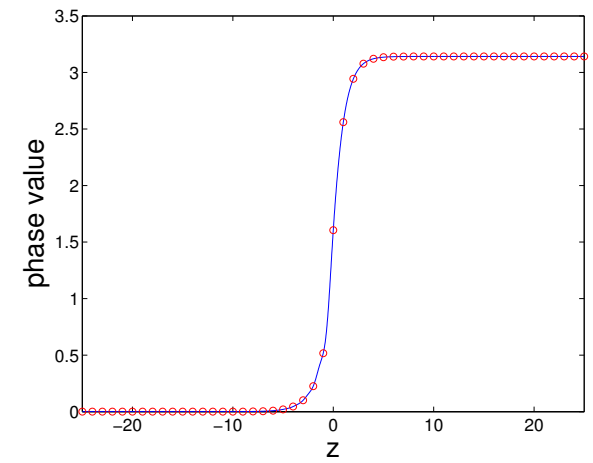

(a)

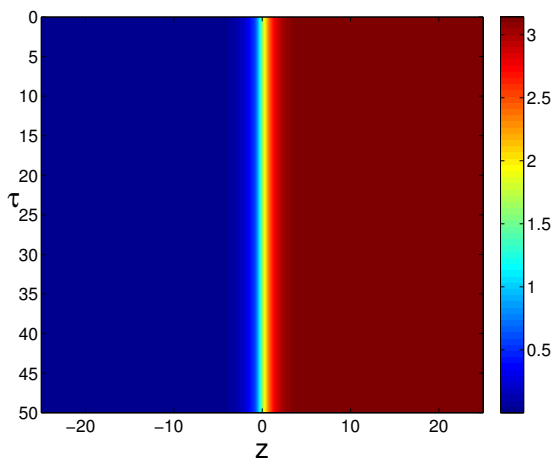

(c)

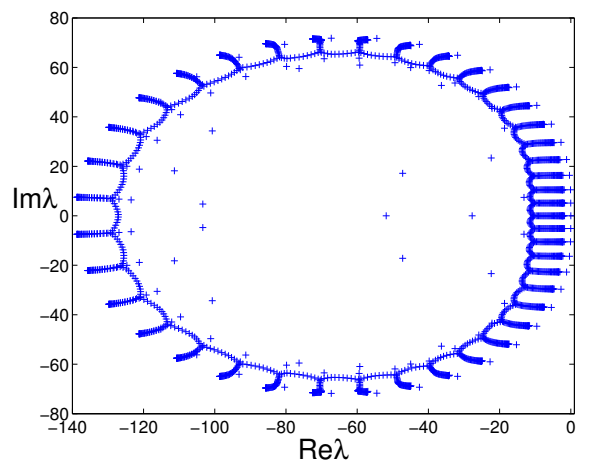

(b)

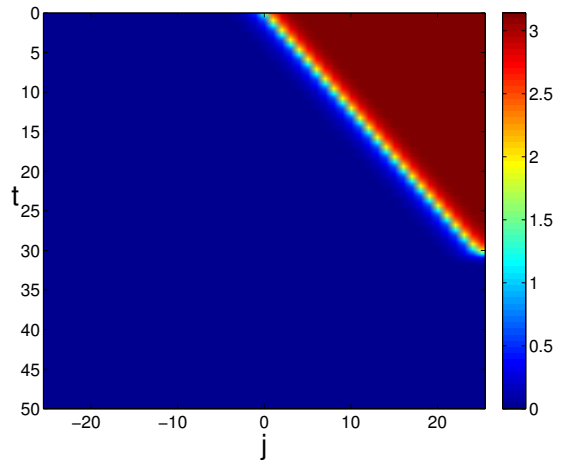

(d)

FIG. 2: (a) Traveling wave solution (solid curve) $\phi(z)$ of (5) at $k=2.25$ and $\mu=0.5$, yielding $c=0.8123$, and the initial condition (circles) $\theta_{j}(0)=\phi(j)$ on the lattice for the ODE simulations shown in (d). (b) Eigenvalues of the Jacobian of the linearization of the co-traveling frame PDE (3) around the stationary solution $\phi(z)$. (c) The space-time evolution of the solution of PDE (3) with the stationary state as the initial condition, $\Theta(z, 0)=\phi(z)$. (d) The space-time evolution of the solution of ODE (1) with initial condition $\theta_{j}(0)=\phi(j)$ shown by circles in (a).

Similar results are shown in Figs. 3 and 4 for the cases of $k=1.5$ and $k=1.1$ at $\mu=0.5$, with traveling wave velocities $c=0.5368$ and $c=0.2382$, respectively, obtained by solving Eq. (5). These are in excellent agreement with the velocities $c=0.5367$ and $c=0.2377$, respectively, obtained from the solution of the ODE system (1) initialized by the traveling wave (d panels). Observe that as $k$ decreases at fixed $\mu$, the velocity $c$ of the traveling wave decreases, and the eigenvalues shown in Figs. 2b, 3b and 4b move closer to the imaginary axis as well. Nonetheless, the obtained eigenvalues in all three cases remain in the left half-plane $\operatorname{Re} \lambda<0$, suggesting that the relevant 
wave is stable as a stationary solution of the co-traveling wave PDE (3). We anticipate that this spectral stability of the solution for the PDE implies the dynamical stability of the traveling wave as the solution of the ODE system (1), as confirmed by our simulations. However, the general demonstration of such a connection is, to the best of our knowledge, an intriguing open problem in analysis.

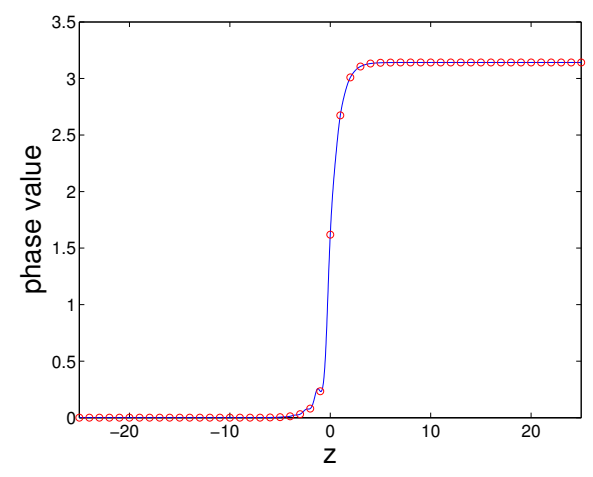

(a)

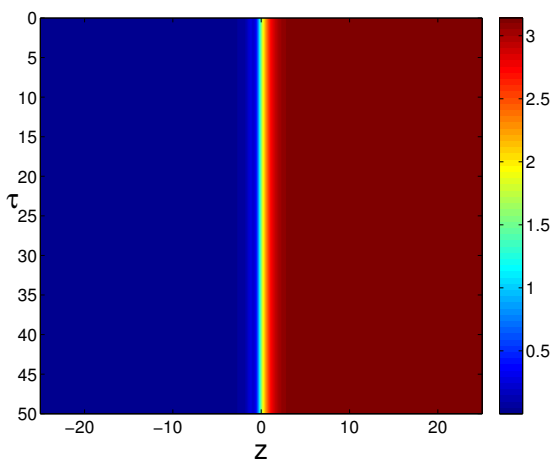

(c)

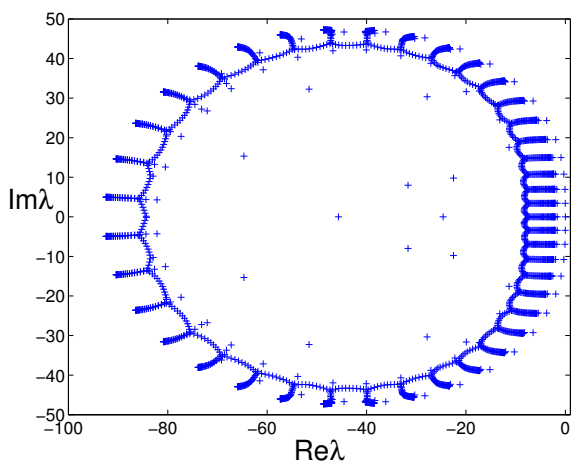

(b)

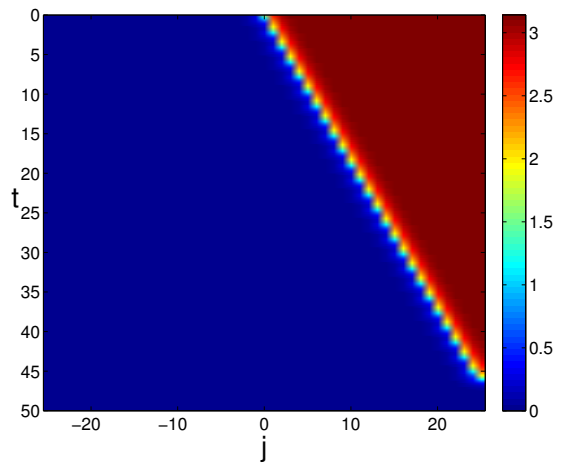

(d)

FIG. 3: Same plots as in Fig. 2 but now for the stable traveling wave solution with $k=1.5$ and $\mu=0.5$, yielding $c=0.5368$.

\section{B. Existence and stability of traveling waves for the chain}

We now explore more systematically the existence and stability of traveling waves in the $(\mu, k)$ parameter plane. The existence results for sufficiently small $\mu(0 \leq \mu \leq 1.5)$ are summarized in Fig. 5a, which shows the contour plot of velocity of the traveling wave computed at different $\mu$ and $k$ using Eq. (5) and complements the steady state analysis of [6] shown in Fig. 1. For comparison, pitchfork (yellow) and saddle-node (red) bifurcation curves for a 51-oscillator chain (and shown by solid and dashed curves, respectively, in Fig. 1) are also included. To obtain the 


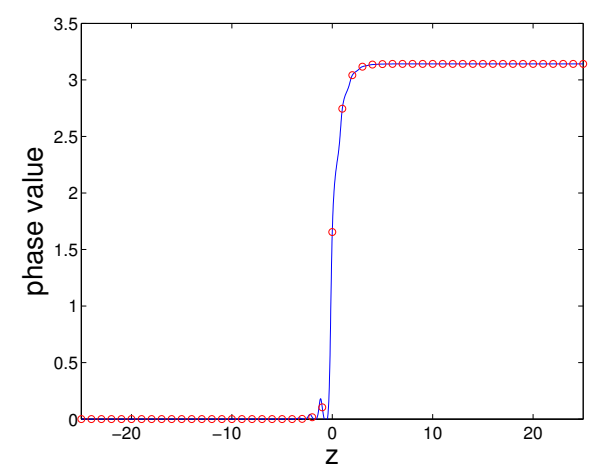

(a)

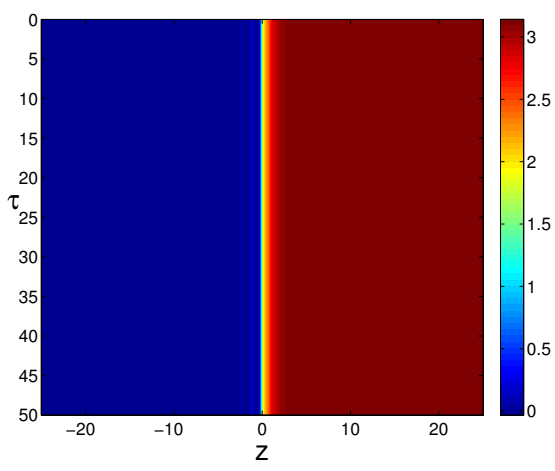

(c)

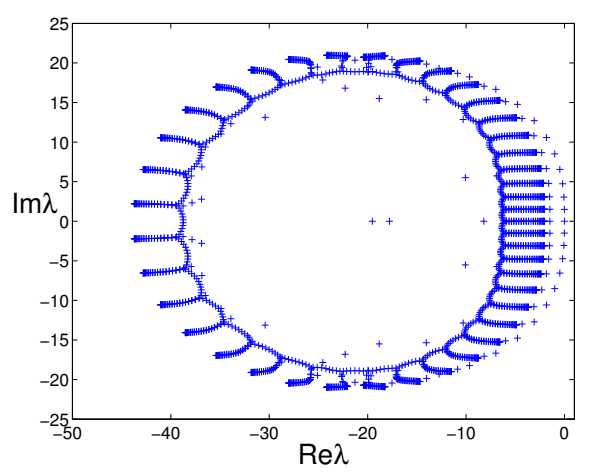

(b)

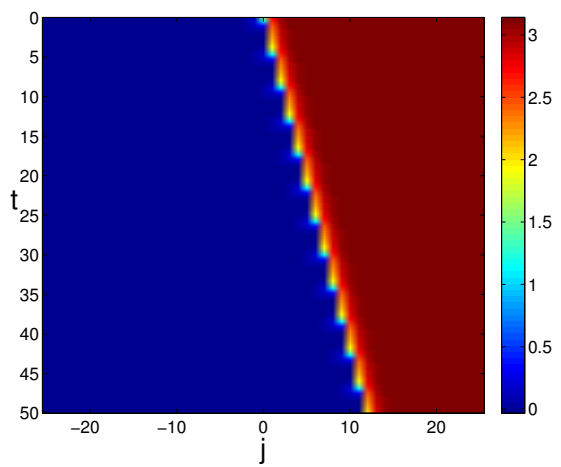

(d)

FIG. 4: Same plots as in Fig. 2 but now for the stable traveling wave solution with $k=1.1$ and $\mu=0.5$, yielding $c=0.2382$.

pitchfork curve, stationary solutions of Eq. (1) are obtained using Newton's method, starting with a value $k=0.4$ for given $\mu$ and using continuation in $k$ until the Jacobian of the relevant system becomes singular. To obtain the saddle-node curve, a stable stationary solution is identified in region B of Fig. 1. This is obtained by integrating Eq. (1) using an unstable solution initial condition over a long time horizon until a stable stationary solution is obtained (as an attractor of the dynamics). This solution is used in Newton's method as a good initial guess in order to obtain the stable waveform within region B. Numerical continuation is then used with increasing $\mu$ until the Jacobian becomes singular. This monoparametric process is repated for different values of $k$ within region $\mathrm{B}$. The curves obtained are almost indistinguishable from the ones in Fig. 1. While we perform our computations in a finite chain, having examined chains of different sizes, we expect our principal conclusions to persist (essentially without modification) for the case of the infinite chain. On the flip side, we can identify traveling waves only in region $\mathrm{C}$, while below it their speed degenerates to 0 , leading to standing waves (split-state equilibria), in agreement with the findings 


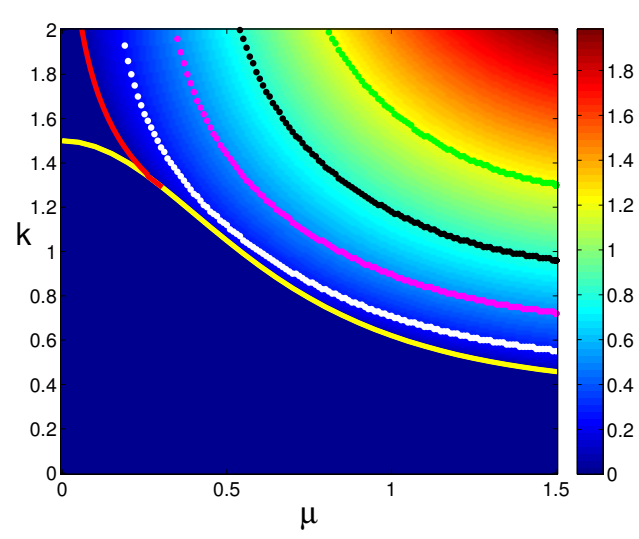

(a)

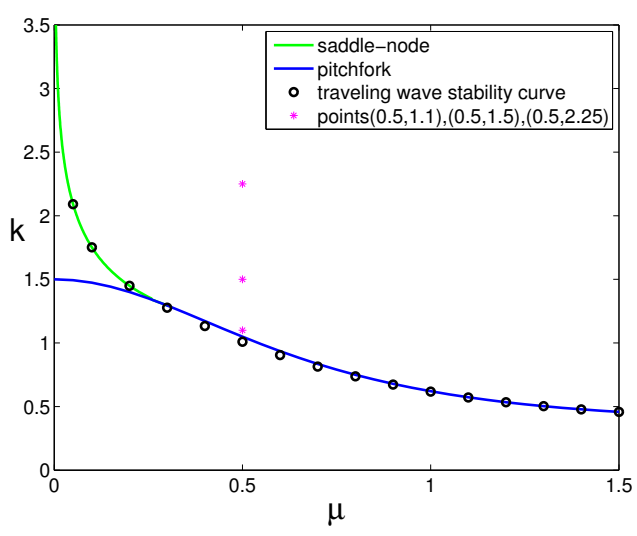

(b)

FIG. 5: (a) Contour plot of the velocity $c$ of the traveling waves in the $(\mu, k)$ plane obtained by solving Eq. (5) and isocontours of $c=0.25$ (white), $c=0.5$ (magenta), $c=0.8$ (black) and $c=1.2$ (green), shown together with the pitchfork (yellow) and saddle-node (red) bifurcation curves obtained for the equilibrium states of the 51-oscillator chain. (b) The curve (open circles) where the traveling waves have (almost) zero velocity nearly coincides with the saddle-node (green) and pitchfork (blue) bifurcation curves; parameter values used in Figures 2,3,4 are also shown (magenta dots).

of [6] for the finite chain case (and our discussion of standing wave states above).

We now explore this comparison in more quantitative detail in Fig. 5b. Here the open circles mark the curve above which numerical simulations of ODE system (1) yield stable traveling waves. To obtain these points, we fixed $\mu$ and solved (5) for the traveling wave solution $\phi(z)$ and its velocity at $(\mu, k)$ starting with $k$ just above the bifurcation curves and then progressively decreasing its value. To verify the velocity of the traveling wave at given $(\mu, k)$, we then used $\theta_{j}(0)=\phi(j)$ as initial conditions in the simulations of (1) and computed the velocity of the propagating front. The open circles were obtained by finding the values of $k$ where the traveling wave solution has zero velocity up to the numerical error in both methods. The comparison strongly suggests that the disappearance of standing wave solutions of [6] gives rise to the traveling wave solutions analyzed here.

The isocontours associated with different velocities, shown in Fig. 5a, illustrate how the velocity $c$ of traveling waves, as obtained from the PDE, depends on the two parameters $\mu$ and $k$. To complement these results with a monoparametric visualization, we also show some plots of $c(k, \mu)$ at fixed $\mu$ in Fig. 6a,b and of $c$ as a function of $\mu$ at fixed $k$ in Fig. 6c,d. These curves show that the velocity increases with $k$ and $\mu$. As the velocity decreases, these curves approach the region containing the standing waves. 


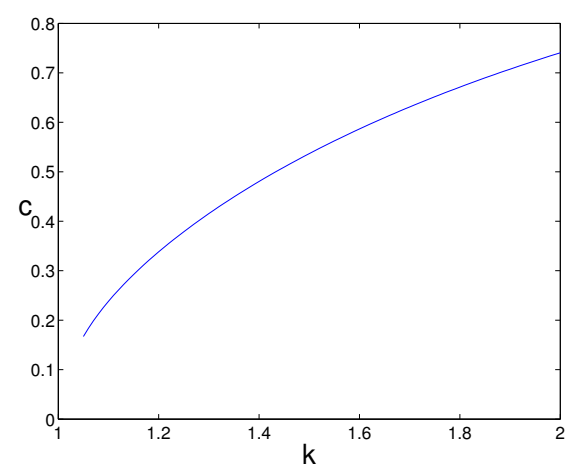

(a) $\mu=0.5$

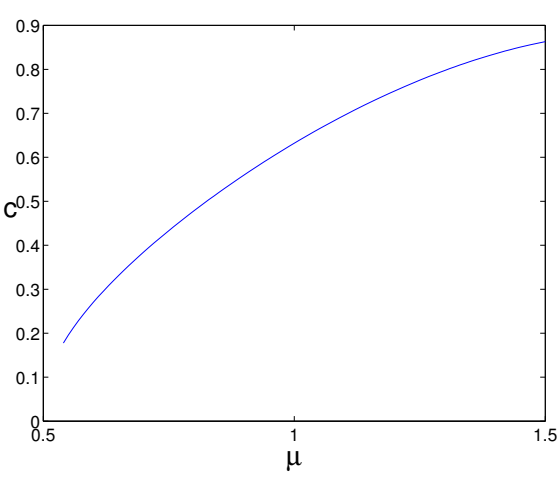

(c) $k=1.01$

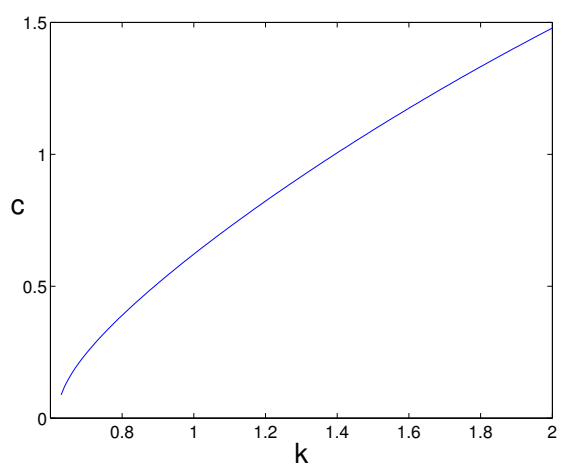

(b) $\mu=1$

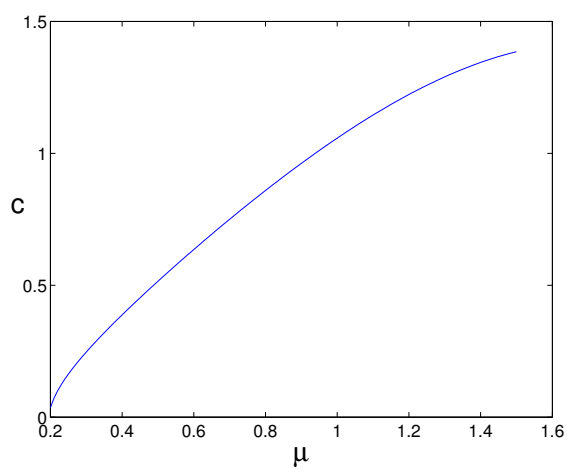

(d) $k=1.46$

FIG. 6: Velocity $c$ as a function of $k$ at fixed $\mu$ (panels a and b) and as a function of $\mu$ at fixed $k$ (panels c and $\mathrm{d})$.

We now discuss an important aspect of the obtained spectral pictures (e.g. the ones shown in Figs. 2b, 3b, 4b) that concerns stability of the background state with $\Theta_{0}=0$ or $\Theta_{0}=\pi$. Our solutions clearly have support on both of these states, so the spectrum of these homogeneous states should be mirrored within that of our (standing or traveling) waves. Substituting $\Theta(z, \tau)=$ $\Theta_{0}+\epsilon v(z, \tau)$ in Eq. (3), where $\epsilon$ is a small parameter, and linearizing about $\Theta_{0}$, one obtains the following linear advance-delay partial differential equation with constant coefficients for $v(z, \tau)$ :

$$
v_{\tau}-c v_{z}=k \cos (\mu)(v(z+1, \tau)-2 v(z, \tau)+v(z-1, \tau))-2 v(z, \tau) .
$$

Seeking solutions of (6) in the form $v=e^{\lambda \tau} e^{i p z}$ for real $p$, we have

$$
\lambda=-2\left(2 k \cos (\mu) \sin ^{2}(p / 2)+1\right)+i c p .
$$

The equilibrium state $\Theta(z, \tau) \equiv \Theta_{0}$ is unstable when $\operatorname{Re} \lambda>0$, i.e. when

$$
k \cos (\mu)<-\frac{1}{2 \sin ^{2}(p / 2)}
$$




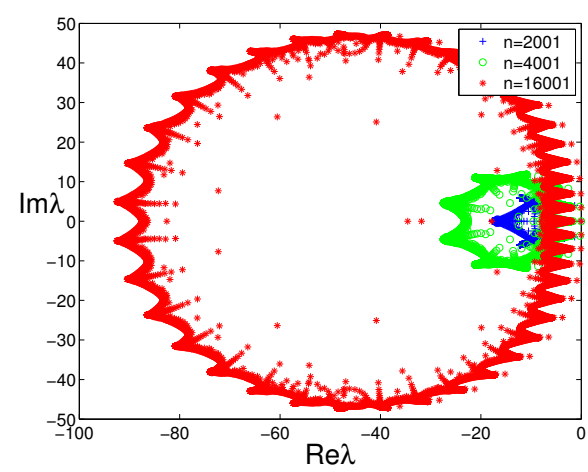

(a)

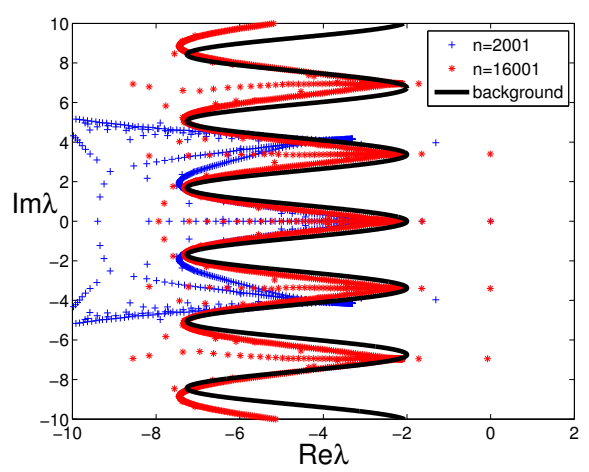

(b)

FIG. 7: (a) The eigenvalues of the Jacobian for the traveling wave solution at $\mu=0.5, k=1.5$ discretized on the interval $[-200,200]$ with $n=2001,4001$ and 16001 nodes. (b) The enlarged version of the $n=2001$ and $n=16001$ cases from (a), along with the continuous background spectrum curve given by Eq. (9), near the imaginary axis.

for some real $p$. Since $k>0$, this can happen for example at large enough $k$ and $\frac{\pi}{2}<\mu<\frac{3 \pi}{2}$. More precisely, the background solution is always unstable for

$$
k>\frac{1}{2}|\sec (\mu)|, \quad \cos (\mu)<0 .
$$

Additionally, since (7) implies that $\operatorname{Re} \lambda=-2\left(2 k \cos (\mu) \sin ^{2}(p / 2)+1\right)$ and $\operatorname{Im} \lambda=c p$, these eigenvalues determine the following locus of points in the spectral plane:

$$
\operatorname{Re} \lambda=-2\left\{2 k \cos (\mu) \sin ^{2}\left(\frac{\operatorname{Im} \lambda}{2 c}\right)+1\right\},
$$

which should be traceable in the linearization spectra of the traveling waves considered here.

For $0 \leq \mu \leq \frac{\pi}{2}$ and $\frac{3 \pi}{2} \leq \mu \leq 2 \pi$, based on equation (9), the background is always stable, so any instability must come from the front itself. For example, Fig. 7a shows the eigenvalues of the Jacobian matrix associated with the traveling wave solution at $\mu=0.5$ and $k=1.5$. The eigenvalues are given for $n=2001,4001$ and 16001 nodes in our discretization of the co-traveling wave problem (3) on the interval $[-200,200]$, with the step size $h=400 /(n-1)$. The eigenvalues of the linearized equation about the background solutions $\Theta_{0}=0$ and $\Theta_{0}=\pi$ are given by Eq. (7). For these parameter values, we theoretically have $-7.27 \leq \operatorname{Re} \lambda \leq-2$. In Fig. 7 one can see a band of eigenvalues folding towards the imaginary axis as $n$ increases. Zooming in on the region near the imaginary axis in Fig. 7b, we see that the eigenvalues are converging to the background spectrum as the continuum limit is approached, as predicted. In the Appendix, we explain further how the forward difference scheme we use results in the particular structure of the continuous spectrum 
of the problem (inducing the parabolic shape observed in Fig. 7a) and how a centered difference scheme would affect the corresponding spectral and stability picture. Similar results are found for the cases of $k=2.25$ and $k=1.1$ (not shown here).

In the above discussion, we extended the analysis of the standing waves in [6] to traveling wave solutions. However, somewhat in line with the considerations in [6], this study until now was limited to $0 \leq \mu \leq 1.5$. We now extend our analysis to the entire upper half of the $(\mu, k)$ plane. So far, we have obtained stability curves (pitchfork and saddle-node) separating traveling and standing waves. Extending these notions to a broader $\mu$-interval in the $(\mu, k)$ plane, we obtain the bifurcation curves for $0 \leq \mu \leq 10$ as shown in Fig. 8. Along with extended pitchfork and saddle-node curves we show the curves above which the background is unstable, according to the inequality (8) obtained above for the linearized problem. The curve separating standing and traveling waves coincides with the saddle-node bifurcation curves where they exist and follows the pitchfork bifurcation curve otherwise.

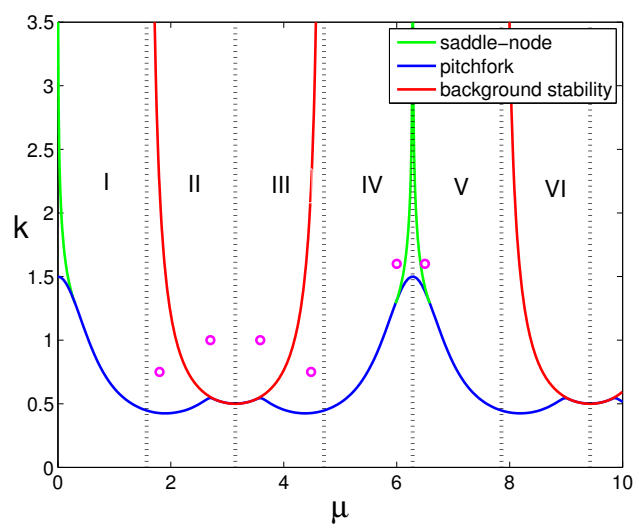

FIG. 8: Extended pitchfork (blue) and saddle-node (green) bifurcation curves and the background instability curve (red). Magenta circles denote the parameter values where the stability of the traveling waves was probed via direct numerical computations.

To explore the stability of traveling wave solutions where they exist, we divide the parameter space into several regions corresponding to different intervals of $\mu$. These are the $\mu$ regions $I$ $\left(\left[0, \frac{\pi}{2}\right]\right), I I\left(\left[\frac{\pi}{2}, \pi\right]\right), I I I\left(\left[\pi, \frac{3 \pi}{2}\right]\right), I V\left(\left[\frac{3 \pi}{2}, 2 \pi\right]\right), V\left(\left[2 \pi, \frac{5 \pi}{2}\right]\right)$ and $V I\left(\left[\frac{5 \pi}{2}, 3 \pi\right]\right)$. In the regions from $I I$ to $V$, we choose points $(\mu, k)$ and solve the traveling wave Eq. (5) for $\phi(z)$ and velocity $c$. Using the forward difference or in some cases the centered difference approximation yields convergence to the relevant solutions. As before, we then use the resulting traveling wave as initial data to solve the ODE system (1) in order to check its stability and visualize the resulting dynamics. To obtain 
the initial condition, we evaluate the traveling wave solution at 51 integer points, and then place it on a grid of 81 points by padding it with 0 s on the left and $\pi \mathrm{s}$ on the right:

$$
\theta_{j}(0)= \begin{cases}0, & j=-40, \ldots,-26 \\ \phi(j), & j=-25, \ldots, 25 \\ \pi, & j=26, \ldots, 40 .\end{cases}
$$

The padding introduces a small perturbation of the initial curve, which, when the solution is unstable, initiates the instability. The points $(\mu, k)=(1.8,0.75),(2.7,1),(2 \pi-2.7,1),(2 \pi-$ $1.8, .75,),(6,1.6)$ and $(6.5,1.6)$ (magenta circles in Fig. 8) are chosen as representative points from the regions $I I, I I I, I V$ and $V$. The points $(\mu, k)=(2.7,1)$ and $(2 \pi-2.7,1)$ lie in the region where the background state was shown to be unstable, above the red curve in Fig. 8. Perturbed traveling waves for these points are shown in Fig. 9 and Fig. 10 respectively.

When $(\mu, k)=(2.7,1) \in I I$, the traveling wave is predicted to move to the right with speed $c=0.2233$. In panel (a) of Fig. 9, the initial condition is shown. In panel (b), the solution is shown at time $t=4$, when the perturbation of the unstable solution has caused the relevant dynamical instability to be manifested in the $\theta \approx 0$ part of the solution. In panel (c), the solution is shown at time $(t=8.5)$, when the instability of the background is manifested at both ends of the domain. Finally, panel (d) gives the space-time contour plot up to time $t=50$, clearly illustrating the destabilization of the background and where it is initiated.

In Fig. 10, when $(\mu, k)=(2 \pi-2.7,1) \in I I I$, the front is predicted to move with speed $c=-0.2233$. The results are similar to the ones discussed above and observed in Fig. 9. Observe that the traveling wave solutions $\phi_{1}(z)$ at $(\mu, k)=(2.7,1)$, shown in Fig. 9a, and $\phi_{2}(z)$ in Fig. 10a at $(\mu, k)=(2 \pi-2.7,1)$ exhibit the symmetry $\phi_{2}(z)=\pi-\phi_{1}(-z)$, and their velocities are equal in absolute value and opposite in sign. This symmetry is discussed below. Importantly, in both cases the intervals where the padding is added, and hence slight perturbations are introduced, are exactly where the instability of the background initially manifests itself (in panels (b) and (c)) numerically.

Fig. 11a shows time snapshots of the solution of the ODE system (1) initialized by the traveling wave (solid blue curve) obtained from Eq. $(5)$ at $(\mu, k)=(1.8,0.75)$, which lies in the region $I I$ below the background instability curve. The space-time contour plot of the solution is shown in Fig. 11b. The traveling wave is initially traveling to the right at the positive predicted velocity $c=0.5493$. However, a frontal instability of the traveling wave leads to the formation of two fronts near $t=7.28$ that can be seen at $t=14.56$ (dashed-dotted green curve) and $t=21.85$ 


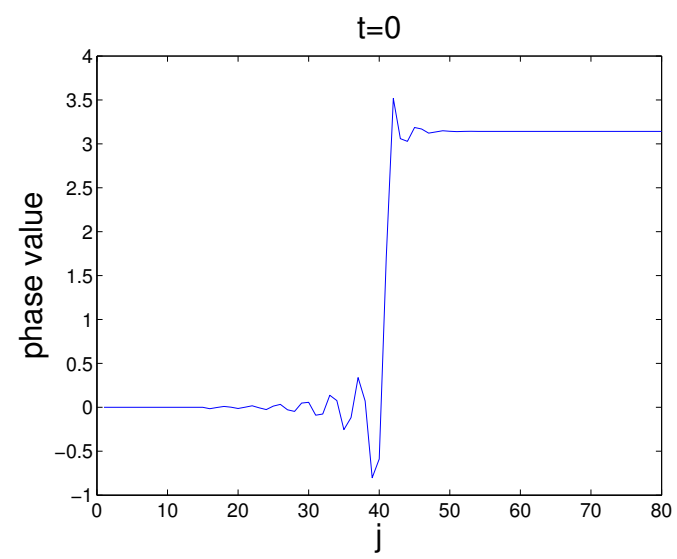

(a)

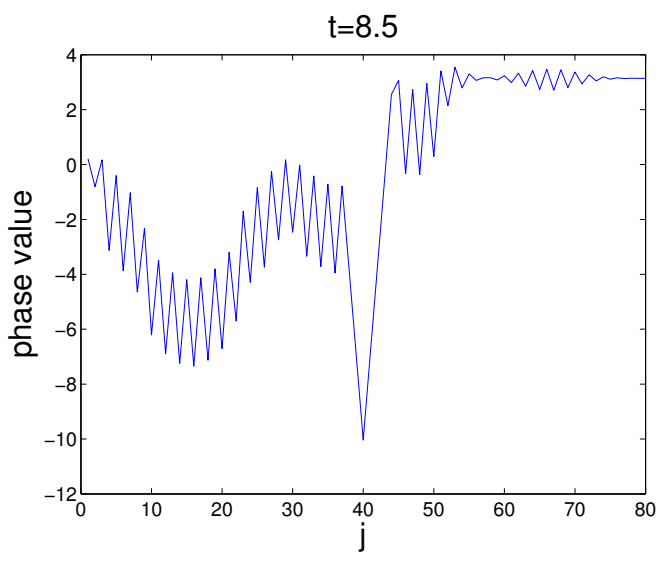

(c)

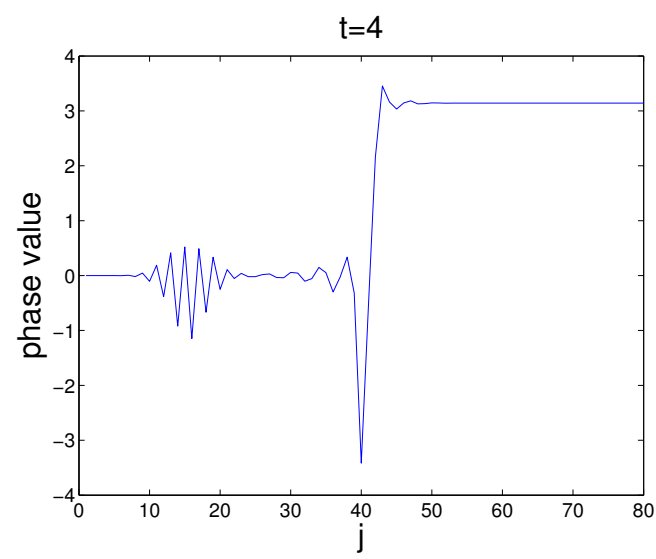

(b)

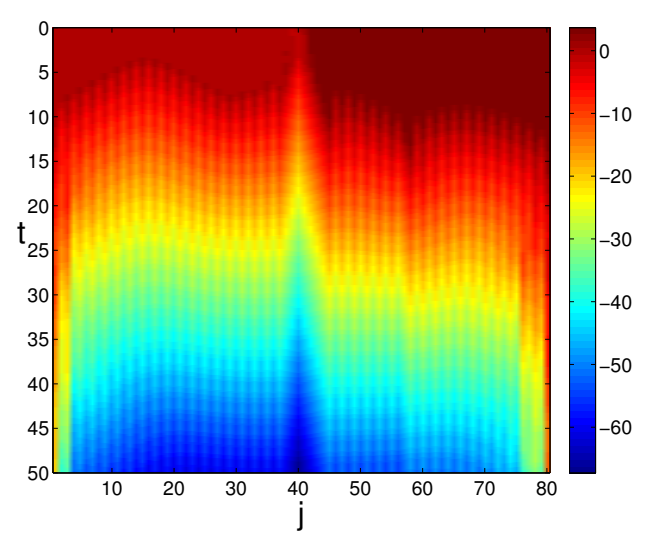

(d)

FIG. 9: Unstable traveling wave at $(\mu, k)=(2.7,1)$ with $c=0.2233$ : (a) Initial state $(t=0)$ obtained from the solution of Eq. (5). (b) Solution of Eq. (1) at $t=4$ where the left side of the solution, with $\theta_{j} \approx 0$, manifests destabilization. (c) Solution at $t=8.5$ where the right side, with $\theta_{j} \approx \pi$, also shows destabilization. (d) Contour plot of space-time evolution until time $t=50$. Here the color code represents $\theta_{j}$ values on the real line, rather than in $[0,2 \pi) \bmod 2 \pi$.

(dotted magenta curve) in Fig. 11a, as well as in the contour plot shown in Fig. 11b. Interestingly, the two fronts propagate in the opposite directions with the same speed as the initial unstable traveling wave. A similar instability is observed at $(\mu, k)=(2 \pi-1.8,0.75)$ in region $I I I$ in Fig. 12 . However, in this case the predicted velocity of the traveling wave is found to be negative, $c=-0.5493$. As explained below, this pair of solutions also exhibits the symmetry mentioned above for the solutions shown in Fig 9a and 10a. In contrast to those solutions, which featured background instability, no instability of the background is observed in these two examples, and only the frontal instability arises. At $k=0.75$, the frontal instability exists at least for $1.65<\mu<q$, where $q$ satisfies $0.75=0.5|\sec (q)|$; that is, until $\mu$ reaches the background instability region. No 


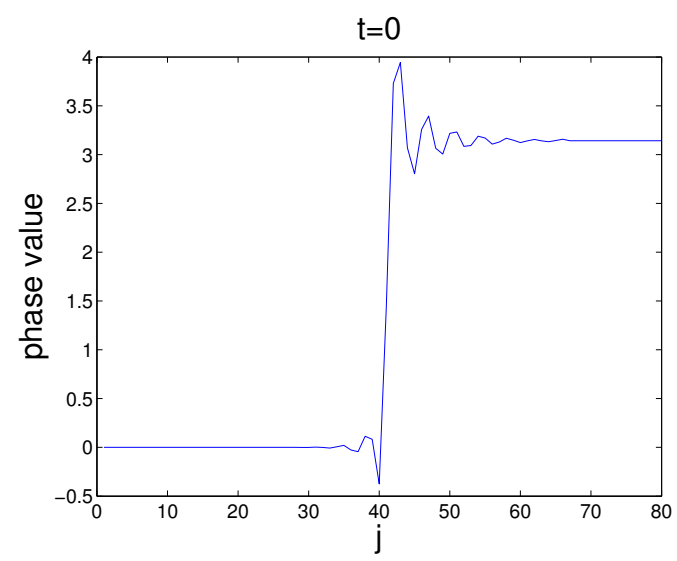

(a)

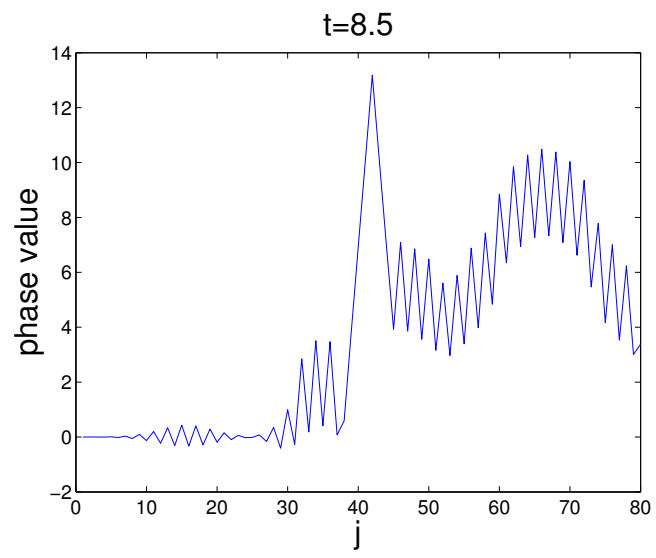

(c)

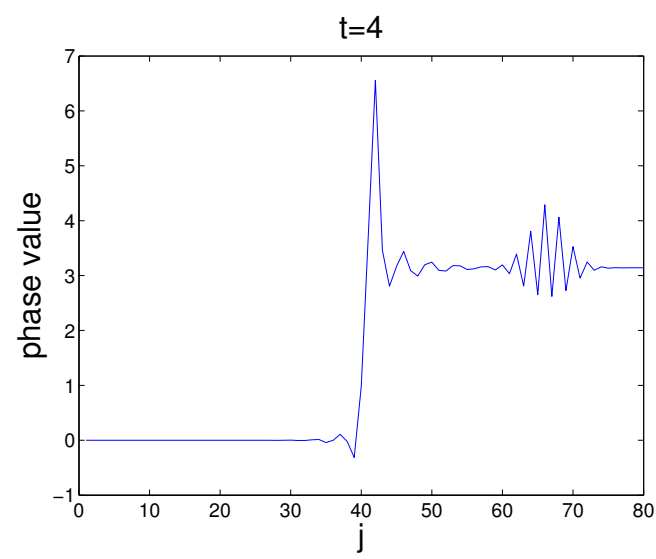

(b)

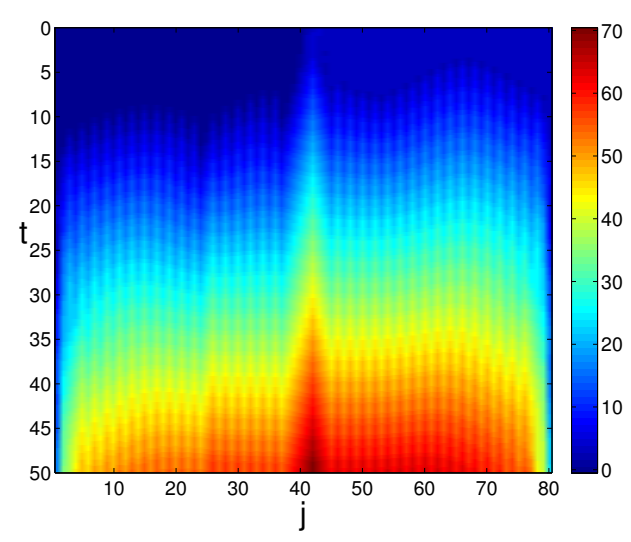

(d)

FIG. 10: Unstable traveling wave at $(\mu, k)=(2 \pi-2.7,1)$ with $c=-0.2233$ : (a) Initial state $(t=0)$ obtained from the solution of Eq. (5). (b) Solution of Eq. (1) at $t=4$ where the right side of the solution, with $\theta_{j} \approx \pi$ imanifests destabilization. (c) Solution at $t=8.5$ where the left side, with $\theta_{j} \approx 0$, suffers a similar effect. (d) Contour plot of space-time evolution until time $t=50$. Again, the color code represents $\theta_{j}$ values on the real line, rather than in $[0,2 \pi) \bmod 2 \pi$.

instability is observed for traveling waves at $\mu \leq 1.65$ and $k=0.75$ propagated until time $t=500$. The results of our stability investigations are summarized in Table I. We emphasize that curves in the $(\mu, k)$-plane separating stable and unstable traveling waves sampled above do not coincide with the vertical lines $\mu=\pi / 2$ and $\mu=3 \pi / 2$ bounding regions II and III where unstable waves were found. In particular, our numerical results indicate that the curve separating stable traveling waves at small $\mu$ from the unstable ones in region II is located in region II slightly to the right of the vertical line $\mu=\pi / 2$. By the symmetry described below we anticipate a similar stability boundary in region III slightly to the left of the line $\mu=3 \pi / 2$.

Recall that the background is stable in the regions $I V$ and $V$. To explore the traveling wave 


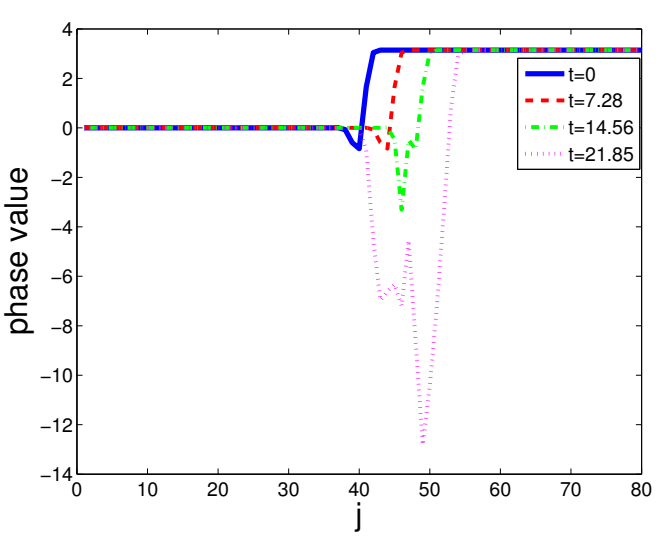

(a)

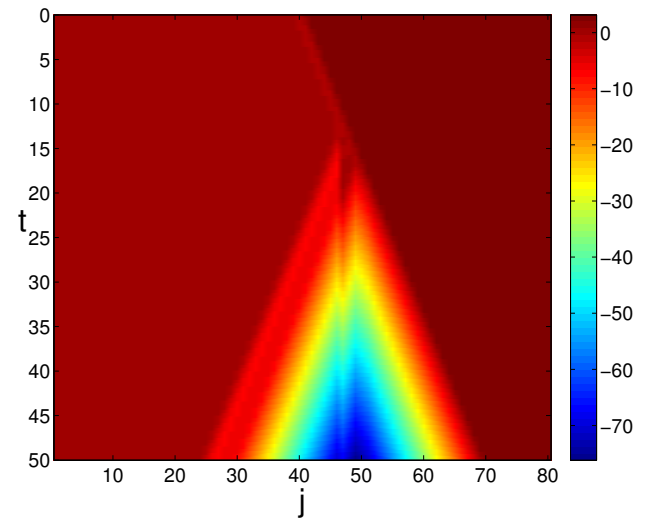

(b)

FIG. 11: Unstable traveling wave at $(\mu, k)=(1.8,0.75)$ with $c=0.5493$ : (a) Snapshots of the solution of Eq. (1) initialized at the traveling wave at times $t=0$ (solid blue curve), 7.28 (approximate onset of instability, dashed red), 14.56 (dash-dotted green) and 21.85 (dotted magenta). (b) The contour plot of space-time evolution of the solution (with $\theta \in \mathbb{R}$ ) until time $t=50$. The traveling wave becomes unstable after an initial transient propagation period and splits into two fronts propagating in the opposite directions with the same speed as the initial wave.

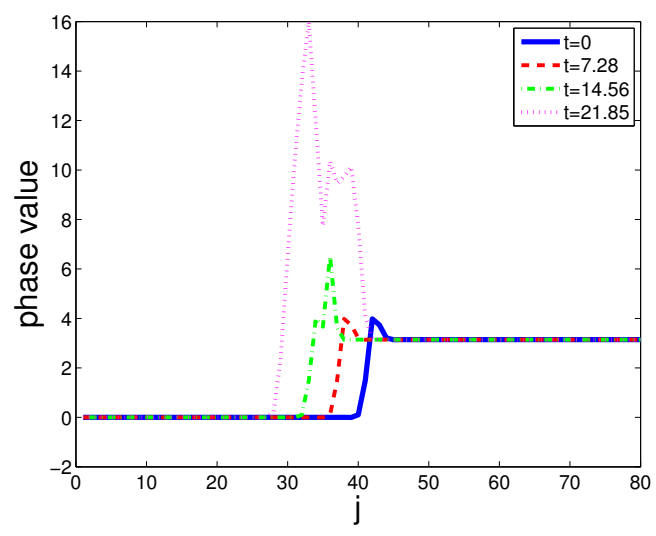

(a)

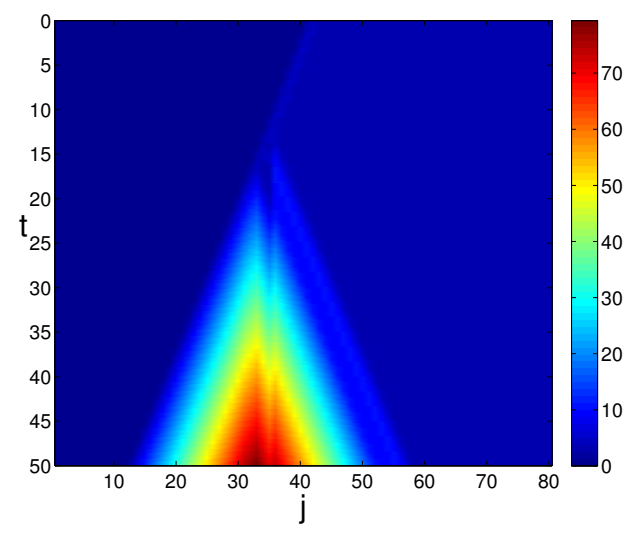

(b)

FIG. 12: Unstable traveling wave at $(\mu, k)=(2 \pi-1.8,0.75)$ with $c=-0.5493$ : (a) Snapshots of the solution of Eq. (1) initialized at the traveling wave at times $t=0$ (solid blue curve), 7.28 (dashed red), 14.56 (approximate onset of instability, dash-dotted green) and 21.85 (dotted magenta). (b) The contour plot of space-time evolution of the solution (with $\theta \in \mathbb{R}$ ) until time $t=50$. The traveling wave becomes unstable after an initial propagation transient period and splits into two fronts propagating in opposite directions with the same speed as the initial wave.

stability in these regions, we solve Eq. (5) at points $(6,1.6)$ and $(6.5,1.6)$ in regions $I V$ and $V$, 
respectively. The fronts and their space-time evolution are shown in Fig. 13 for $(\mu, k)=(6,1.6)$ and Fig. 14 for $(\mu, k)=(6.5,1.6)$. At the point $(6,1.6)$ in region $I V$, the wave is stable and travels to the left, in accordance with the negative velocity $c=-0.2919$ predicted by the traveling wave equation; see Fig. 13. At the point $(6.5,1.6)$ in region $V$, the wave is also stable and travels to the right with the positive velocity $c=0.1894$, as shown in Fig. 14 .

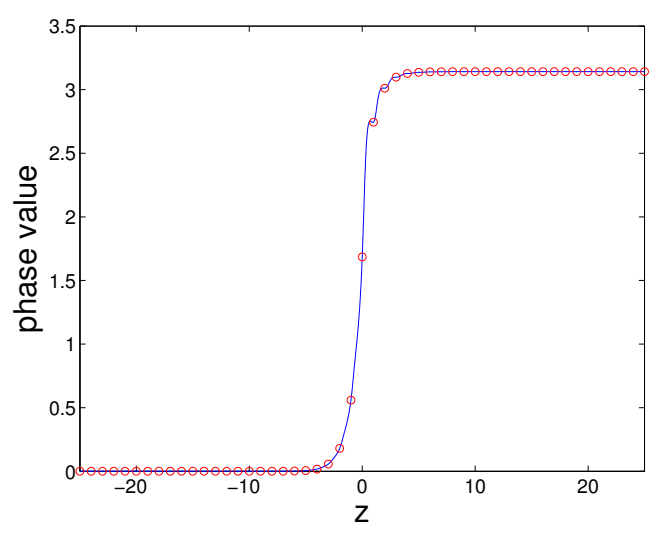

(a)

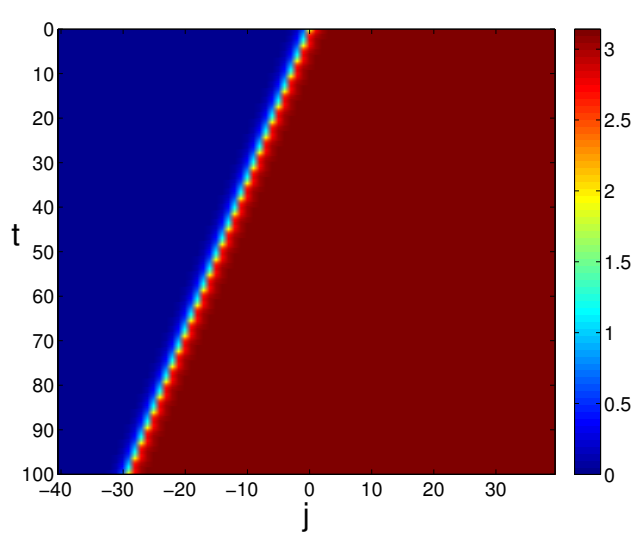

(b)

FIG. 13: Stable traveling wave at $(\mu, k)=(6,1.6)$ with $c=-0.2919$ : (a) The traveling wave $\phi(z)$ (solid line) and the initial condition $\theta_{j}(0)=\phi(j)$ (circles) for the simulation of the ODE system (1). (b) The contour plot of the space-time evolution of the solution of ODE system (1) until time $t=100$. In accordance with the predicted negative velocity, the traveling wave propagates to the left.

TABLE I: Stability and velocities of traveling waves

\begin{tabular}{cccc}
\hline \hline point & region & stability & velocity \\
\hline$(2.7,1)$ & $I I$ & unstable (background) & 0.2233 \\
$(2 \pi-2.7,1)$ & $I I I$ & unstable (background) & -0.2233 \\
$(1.8,0.75)$ & $I I$ & unstable (frontal) & 0.5493 \\
$(2 \pi-1.8,0.75)$ & $I I I$ & unstable (frontal) & -0.5493 \\
$(6,1.6)$ & $I V$ & stable & -0.2919 \\
$(6.5,1.6)$ & $V$ & stable & 0.1894 \\
\hline
\end{tabular}

To extend these results to the whole upper half of the parameter plane, we observe that the right hand side of the traveling wave Eq. (5) is $2 \pi$-periodic in $\mu$, implying that its solutions are also $2 \pi$-periodic in $\mu$. For example, the traveling wave solutions in region $I$ are isomorphic to the ones in region $V$ ( $I I$ with $V I$ etc.). Note also that the traveling (and standing) wave solutions are symmetric about $\mu=\pi$. To show this, we define new variables $\bar{c}=-c, \bar{\mu}=2 \pi-\mu, \bar{z}=-z$, 


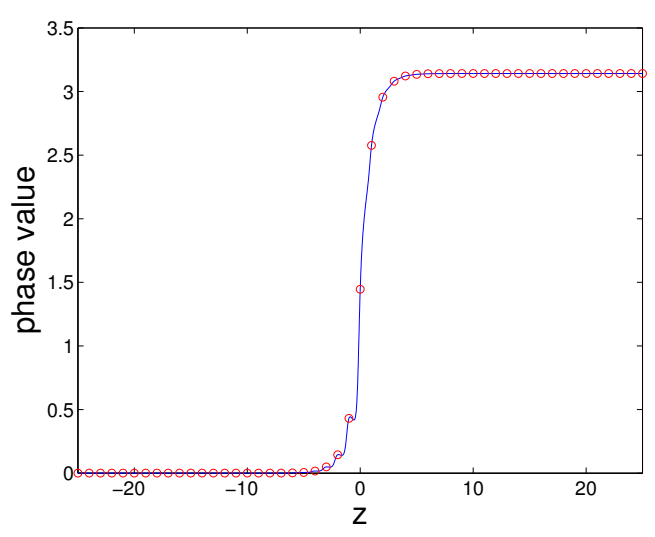

(a)

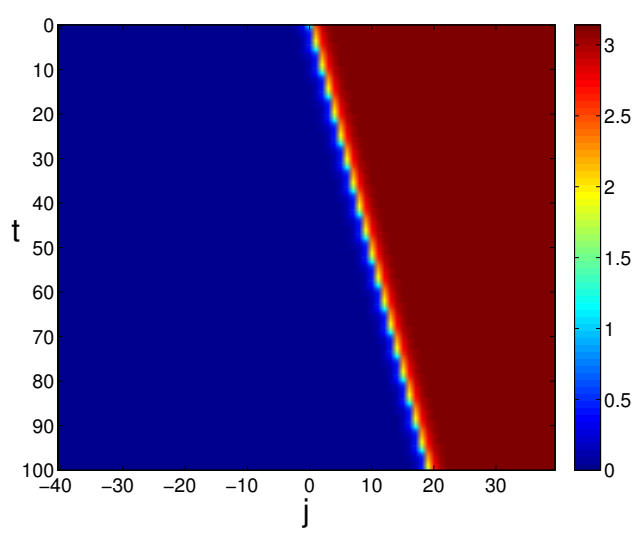

(b)

FIG. 14: Stable traveling wave at $(\mu, k)=(6.5,1.6)$ with $c=0.1894$ : (a) The traveling wave $\phi(z)$ (solid line) and the initial condition $\theta_{j}(0)=\phi(j)$ (circles) for the simulation of the ODE system (1). (b) The contour plot of the space-time evolution of ODE system (1) until time $t=100$. In accordance with the predicted positive velocity, the traveling wave now propagates to the right.

$\bar{\phi}(\bar{z})=\pi-\phi(z)$. This yields $\phi^{\prime}(z)=\bar{\phi}^{\prime}(\bar{z}), \phi(z+1)=\pi-\bar{\phi}(\bar{z}-1)$ and $\phi(z-1)=\pi-\bar{\phi}(\bar{z}+1)$. Substituting this in Eq. (5) for our choice of $H(\theta)$ and $f(\theta)$ we obtain, after simplification,

$$
-\bar{c} \bar{\phi}^{\prime}(\bar{z})=k(\sin (\bar{\phi}(\bar{z}+1)-\bar{\phi}(\bar{z})+\bar{\mu})-\sin (\bar{\mu})+\sin (\bar{\phi}(\bar{z}-1)-\bar{\phi}(\bar{z})+\bar{\mu})-\sin (\bar{\mu}))-\sin (2 \bar{\phi}(\bar{z})),
$$

which in fact coincides with Eq. (5). Hence, $\bar{\phi}(\bar{z})$ is a solution to the traveling wave equation at $\bar{\mu}=2 \pi-\mu$ with velocity $\bar{c}=-c$. In other words, for any $k>0$, given a traveling wave $\phi(z)$ with $\mu=\pi+b$ (for some $b$ ), there is a traveling wave at $\mu=\pi-b$ obtained through the above transformations. This traveling wave at $\mu=\pi-b$ has the same speed and spectrum as the wave with $\mu=\pi+b$ but propagates in the direction opposite to that of the original traveling wave. This implies that the backward moving waves in regions $I I I$ and $I V$ are obtained directly from the waves in regions $I I$ and $I$, respectively, with the spectrum (and hence stability properties) preserved. The observed symmetry obviously exists not only at $\pi$ but at $(2 m-1) \pi$ for any integer $m$. Note that in accordance with these results, the extended curve shown in Fig. 8 repeats periodically once $\mu$ reaches $2 \pi$ and is symmetric about $\mu=\pi$ and $\mu=3 \pi$. Since the waves under study are $2 \pi$-periodic, this analysis can be extended to the entire upper half of the $(\mu, k)$ plane. In particular, it explains the symmetry observed above for the solution pairs at $(2.7,1),(2 \pi-2.7,1)$ and at $(1.8,0.75)$, $(2 \pi-1.8,0.75)$.

In summary, we have obtained an analytical expression for the boundary of a region in parameter space where traveling waves suffer a background instability. Numerical simulations show that as 
parameters are varied within from the zero-speed boundary of the traveling wave region, traveling waves can be stable or can undergo either a background instability (within the region predicted analytically) or a frontal instability (between the stable region and the background instability region). Due to a symmetry in the model corresponding to the periodicity of $H$ and $f$, all results repeat periodically in $\mu$.

\section{Two-dimensional phase equation dynamics}

We now turn to some explorations of the two-dimensional generalization of our oscillator problem. The extension of Eq. (1) to two dimensions has the form

$$
\begin{aligned}
\dot{\theta}_{1,1} & =k\left(H\left(\theta_{1,2}-\theta_{1,1}\right)+H\left(\theta_{2,1}-\theta_{1,1}\right)\right)+f\left(\theta_{1,1}\right) \\
\dot{\theta}_{i, 1} & =k\left(H\left(\theta_{i, 2}-\theta_{i, 1}\right)+H\left(\theta_{i+1,1}-\theta_{i, 1}\right)+H\left(\theta_{i-1,1}-\theta_{i, 1}\right)\right)+f\left(\theta_{i, 1}\right), \quad i=2, \ldots, 2 n-1, \\
\dot{\theta}_{1, j} & =k\left(H\left(\theta_{1, j+1}-\theta_{1, j}\right)+H\left(\theta_{1, j-1}-\theta_{1, j}\right)+H\left(\theta_{2, j}-\theta_{1, j}\right)\right)+f\left(\theta_{1, j}\right), \quad j=2, \ldots, 2 n-1, \\
\dot{\theta}_{i, j} & =k\left(H\left(\theta_{i, j+1}-\theta_{i, j}\right)+H\left(\theta_{i, j-1}-\theta_{i, j}\right)+H\left(\theta_{i+1, j}-\theta_{i, j}\right)+H\left(\theta_{i-1, j}-\theta_{i, j}\right)\right)+f\left(\theta_{i, j}\right), \\
& i, j=2, \ldots, 2 n-1, \\
\dot{\theta}_{i, 2 n} & =k\left(H\left(\theta_{i, 2 n-1}-\theta_{i, 2 n}\right)+H\left(\theta_{i+1,2 n}-\theta_{i, 2 n}\right)+H\left(\theta_{i-1,2 n}-\theta_{i, 2 n}\right)\right)+f\left(\theta_{i, 2 n}\right), \\
& i=2, \ldots, 2 n-1, \\
\dot{\theta}_{2 n, j} & =k\left(H\left(\theta_{2 n, j+1}-\theta_{2 n, j}\right)+H\left(\theta_{2 n, j-1}-\theta_{2 n, j}\right)+H\left(\theta_{2 n-1, j}-\theta_{2 n, j}\right)\right)+f\left(\theta_{2 n, j}\right), \\
& j=2, \ldots, 2 n-1, \\
\dot{\theta}_{2 n, 2 n} & =k\left(H\left(\theta_{2 n, 2 n-1}-\theta_{2 n, 2 n}\right)+H\left(\theta_{2 n-1,2 n}-\theta_{2 n, 2 n}\right)\right)+f\left(\theta_{2 n, 2 n}\right) .
\end{aligned}
$$

We start by solving Eq. (5) on the interval $[-25,25]$ at the parameter values $k=1.3$ and $\mu=0.5$ to obtain a one-dimensional traveling wave $\phi(z)$ with velocity $c=0.4155$. We then solve Eq. (10) for $\theta_{i, j}(t)$ using the classical fourth order Runge-Kutta method with the initial data

$$
\theta_{i, j}(0)= \begin{cases}0, & i \leq 3 \\ \phi(i-3), & 4 \leq i \leq 54 \\ \pi, & i \geq 55,\end{cases}
$$

thus studying the two-dimensional evolution of an initially planar front. The planar front propagates in the horizontal direction at the velocity of the one-dimensional wave, suggesting that the solution $\theta_{i, j}(t)=\phi(i-c t)$, which corresponds to a stable traveling wave in the one-dimensional problem, is also stable in the two-dimensional setting. To illustrate this stability, we distort a 

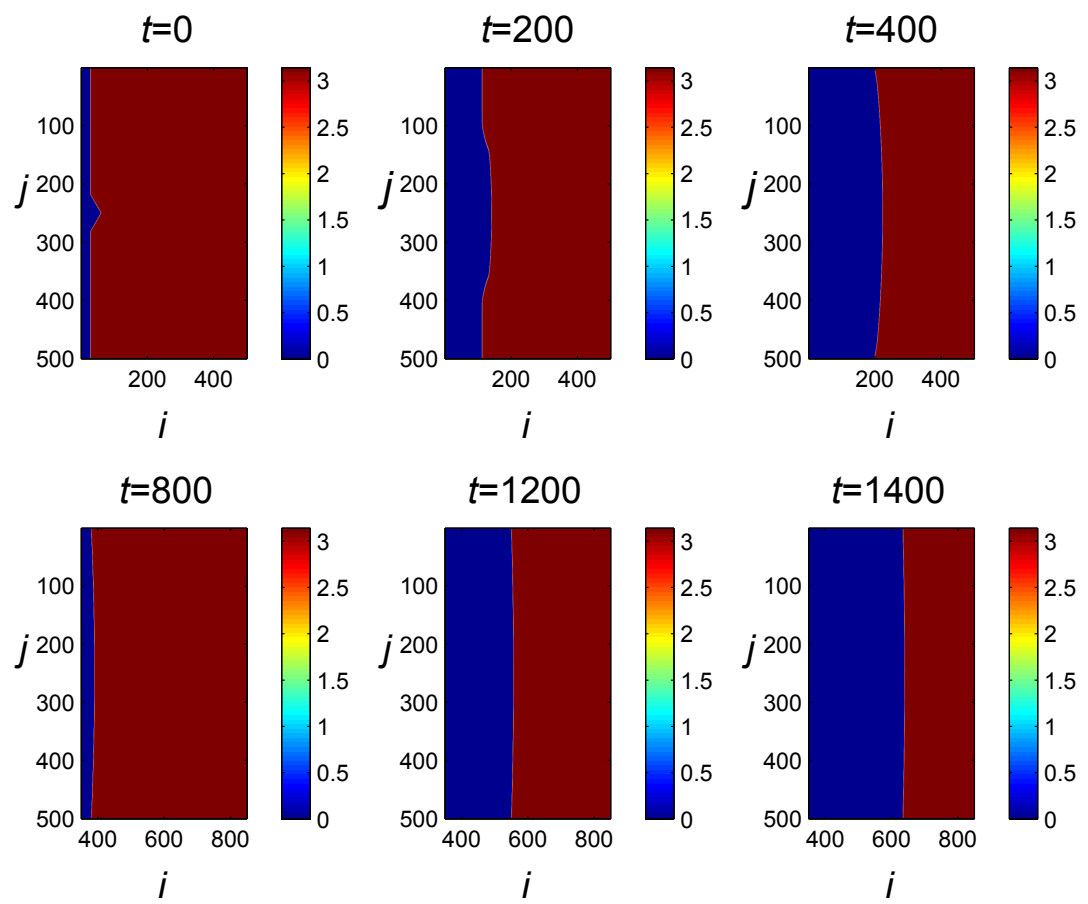

FIG. 15: Snapshots of the evolution of a perturbed planar front introduced within a two-dimensional domain with $k=1.3, \mu=0.5$ at times $t=10,200,400,800,1200$ and 1400. The evolution shows the healing of the front and the decay of the associated perturbation that restore the dynamically robust planar front traveling with velocity $c=0.4155$.

segment of the planar front in the initial condition, as shown in the first panel $(t=0)$ in Fig. 15. The resulting evolution is shown in the remaining panels of Fig. 15, where for better visualization, the range of the $i$-axis is shifted to the left in the last three panels. It can be seen that over time the system "heals" the perturbation and gradually restores its quasi-one-dimensional planar front character, while the solution eventually settles into traveling with the velocity predicted by the one-dimensional results.

Next we consider the evolution of a radial front. In the first four panels of Fig. 16, we show the evolution of an initially circular front for $k=1.3$ and $\mu=0.5$. The initial condition is set to $\theta_{i, j}(0)=\pi$ for $(i, j)$ within the circle of radius 30 centered at $(40,40)$, and $\theta_{i, j}(0)=0$ outside of the concentric circle of radius 36 . The initial value of $\theta_{i, j}$ for $(i, j)$ between these circles is obtained by linear interpolation. The front shrinks and is eventually annihilated (i.e., disappears). The bottom panel of Fig. 16 displays horizontal slices of the solution at the initial and final time steps shown above. Notice that in this case, apparently, the initial radial profile of the front is gradually 

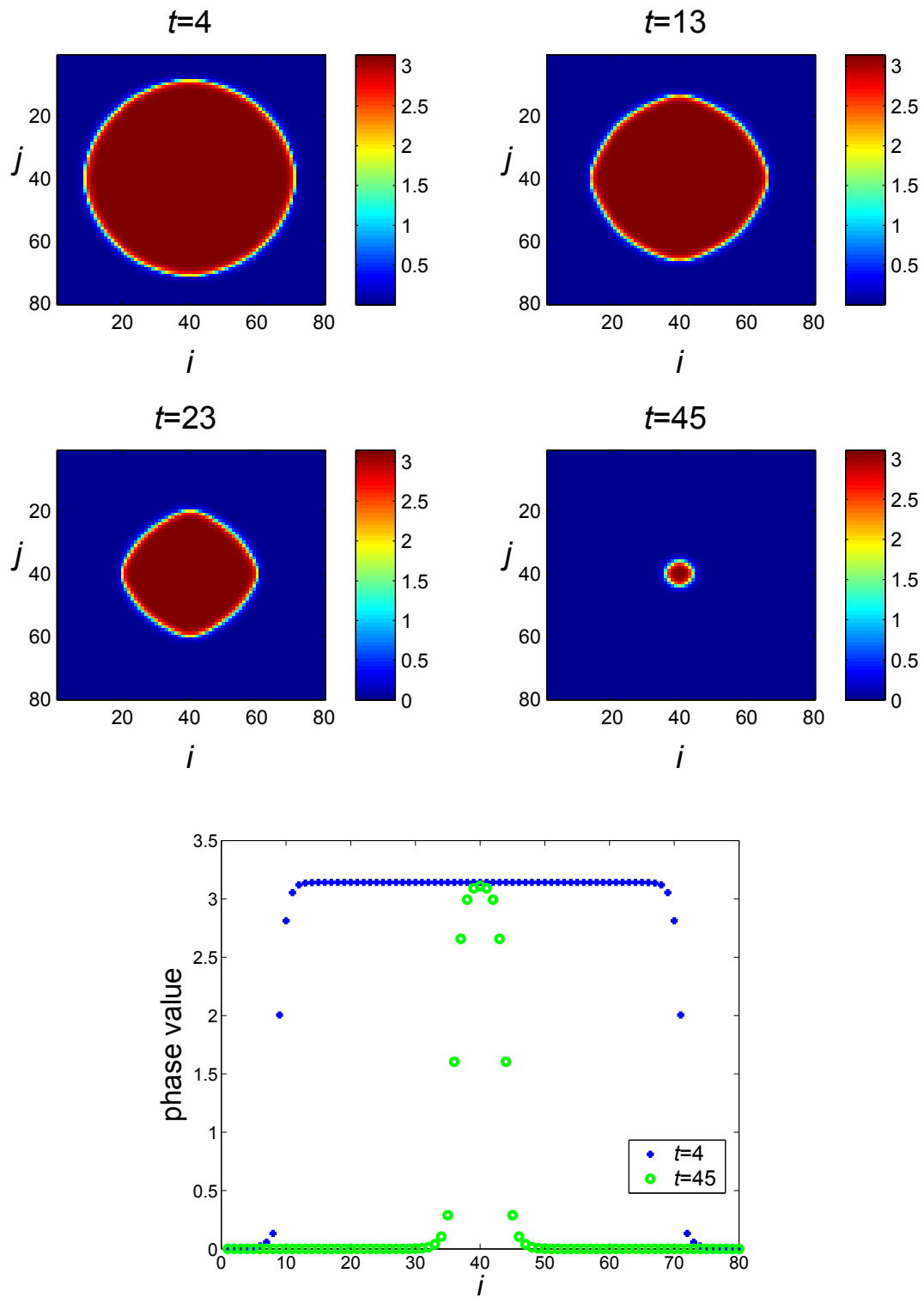

FIG. 16: Snapshots of the evolution of a circular front with $k=1.3, \mu=0.5$ at times $t=4,13,23$ and 45. The front shrinks (and is eventually annihilated) as time evolves. In the bottom panel, a horizontal $\operatorname{section}(j=40)$ of the front at $t=4$ is denoted by plus signs and a horizontal section of the front at $t=45$ (of smaller width) is denoted by circles.

deformed to conform more suitably to the square symmetry of the underlying lattice grid. Hence it appears that linear (planar) fronts are fairly robust in this system, while radial ones are clearly not as robust and eventually disappear.

As noted in [5], Carpenter [4] observed that experimentally induced phosphenes move according to the following rules in two dimensions: 
1. Lines never cross through one another. Rather, they combine to form loops.

2. A line never breaks apart unless it meets another line.

To test whether our two-dimensional model captures these features, we now consider simulations with two symmetric fronts that initially bulge either outward (Fig. 17 and Fig. 19) or inward (Fig. 18 and Fig. 20). These initial conditions are shown in the first panel $(t=0)$ of each figure. The simulation results shown in Figs. 17-20, with $k=1.3, \mu=0.5$ in Fig. 17 and Fig. 18 and $k=2$, $\mu=0.15$ in Fig. 19 and Fig. 20, are consistent with Carpenter's observations listed above. We can see that the outwardly bulging fronts eventually touch near the edges of the domain and form one loop. Meanwhile, the inwardly bulging fronts eventually touch near their centers and the lines break into two parts. These features are very much in line with the expectations of [5] (compare with their Fig. 3). However, it should also be mentioned that [5] posit a third and final feature, namely that:

3. Neighboring lines show a tendency to move in a similar manner.

Our simulations of the coupled oscillator model did not reveal such a tendency. Whether the model can be improved to reflect this feature is a question that remains to be considered in future studies.
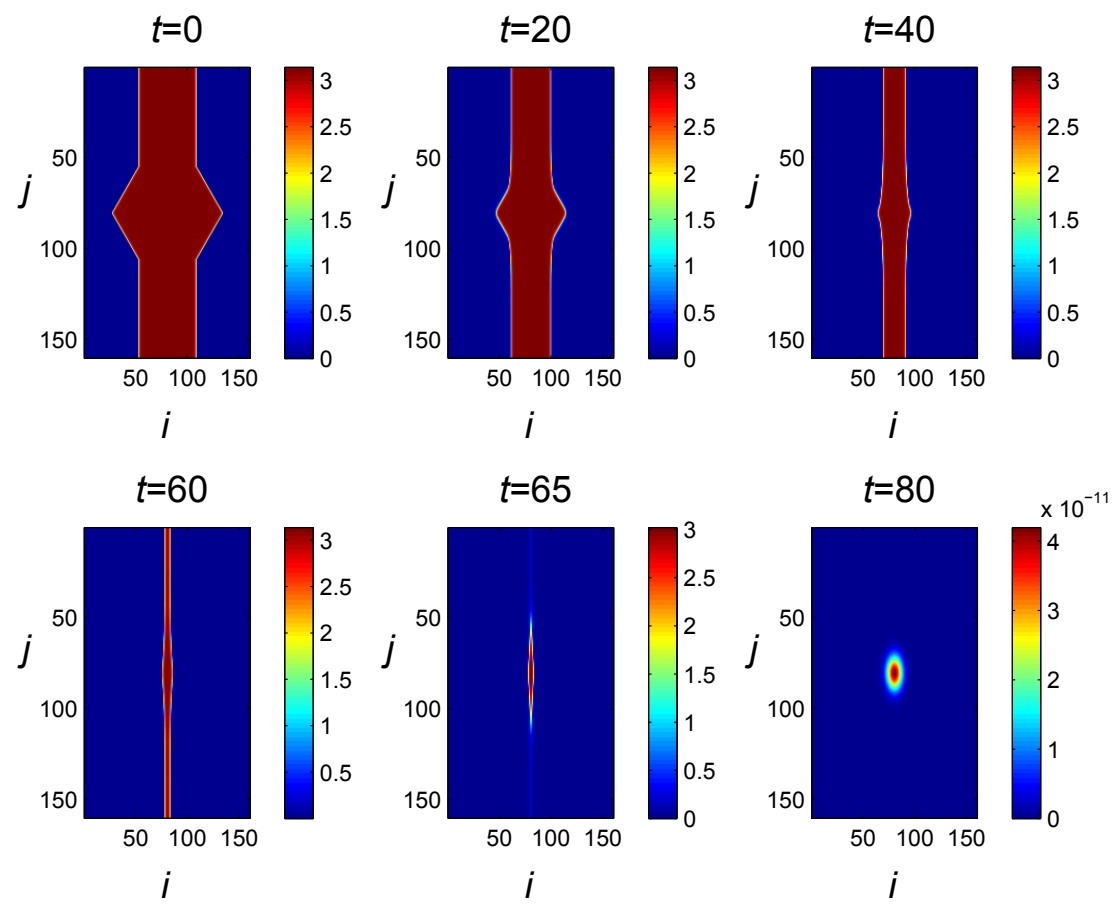

FIG. 17: Snapshots of two outwardly perturbed fronts at times $t=0,20,40,60,65$ and 80 when $k=1.3$, $\mu=0.5$. The fronts meet near the domain edges, form a loop, and shrink. 

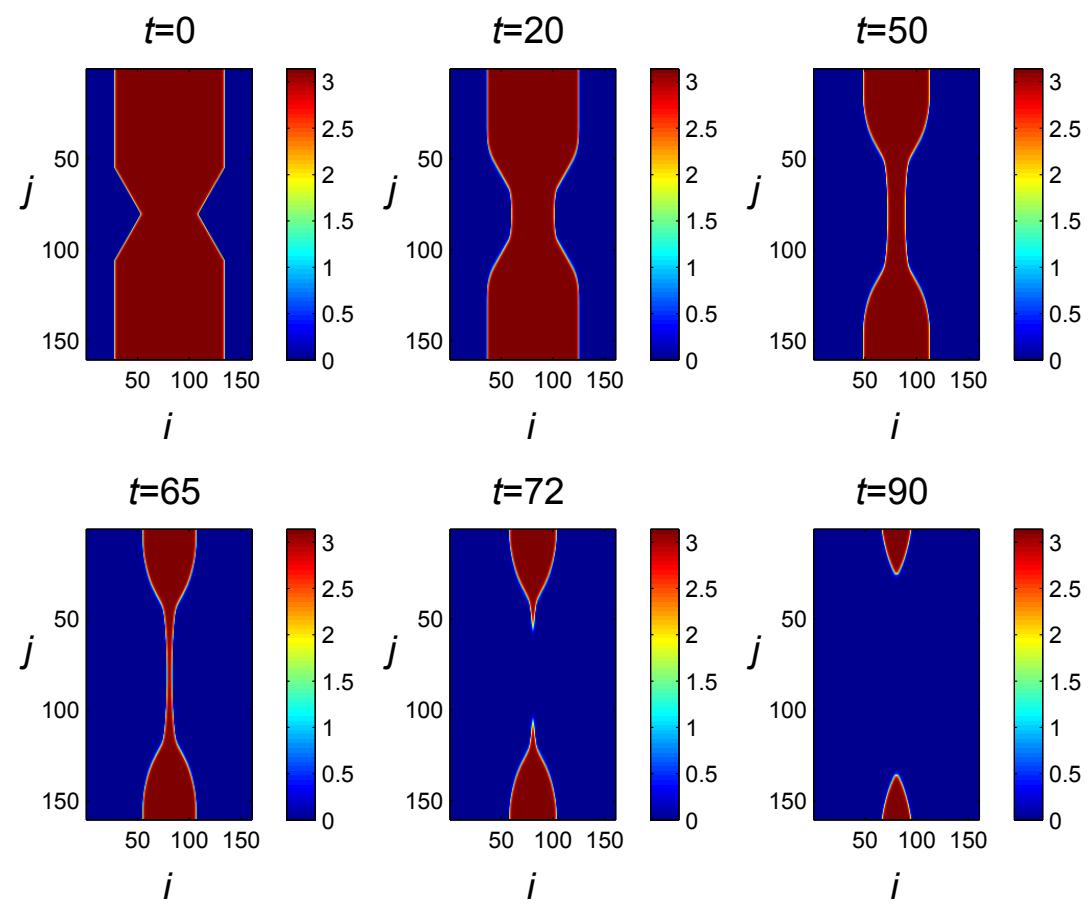

FIG. 18: Snapshots of two inwardly perturbed fronts at times $t=0,20,50,65,72$ and 90 when $k=1.3$, $\mu=0.5$. The fronts meet near their centers, separate, and evolve into two (upper and lower) parts.

\section{CONCLUSIONS AND FUTURE CHALLENGES}

In the present work we have revisited a generic nonlinear lattice model derived in [6] and associated with the dynamics of a forced network of coupled oscillators. A specific motivation for studying this system comes from its relevance to phosphenes, artificial perceptions of light arising in the visual system in which contours, possibly representing boundaries between sets of neurons in different activity states or phases, emerge and propagate. We complemented the important initial steady state analysis of [6] (see also [5]) by exploring the possibility of traveling waves in the system of ordinary differential equations. This led us to introduce the co-traveling frame (advance-delay) PDE and study existence and stability properties of the traveling waves as both special periodic modulo shift solutions of the original systems of ODEs and stationary solutions of the co-traveling frame PDE. Our results on traveling waves complement the work of [6] on the existence and stability of standing waves. We found a curve above which the standing waves become unstable. This curve agrees with and extends the bifurcation curves obtained in [6] by analyzing the equilibrium states of the ODE system. We showed that the instability of standing waves above 

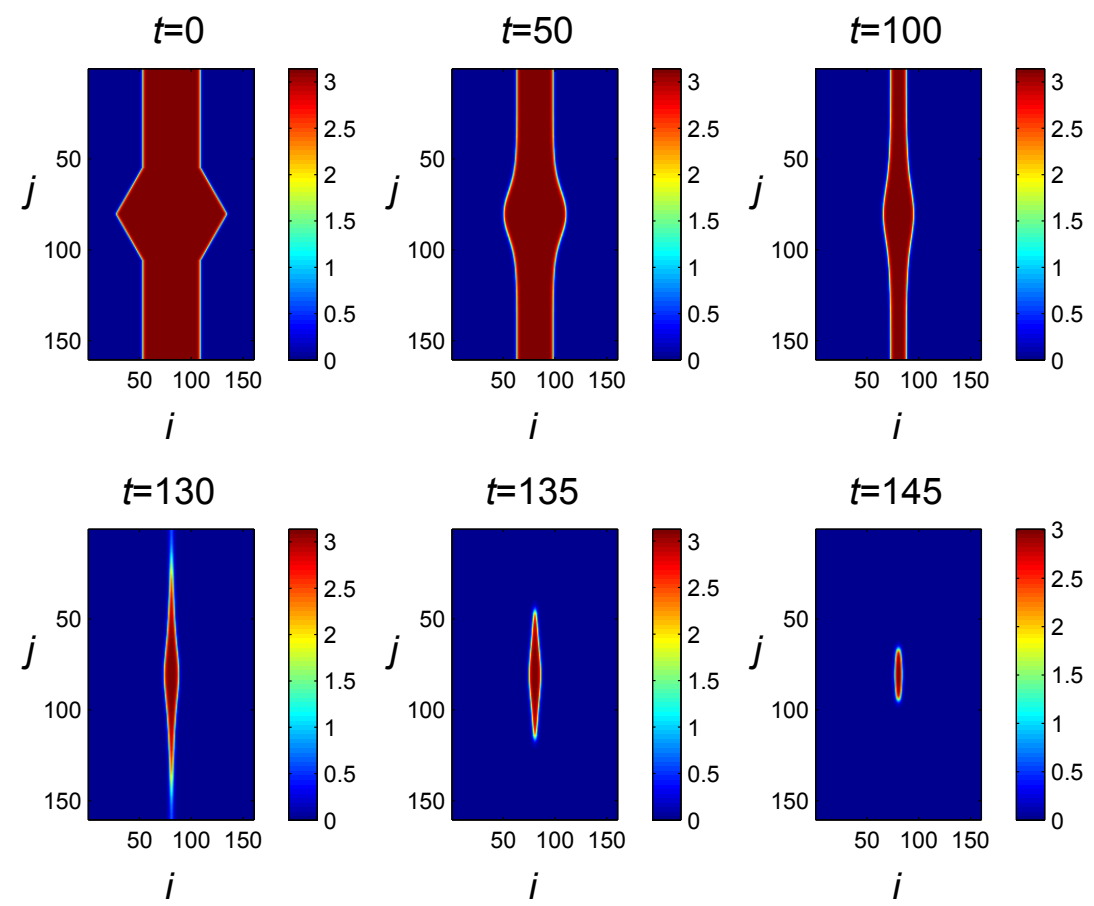

FIG. 19: Snapshots of two outwardly perturbed fronts at times $t=0,50,100,130,135$ and 145 when $k=2$, $\mu=0.15$. As in Fig. 17, the fronts meet near the domain edges, form a loop, and shrink.

the curve leads to the emergence of stable traveling waves in some parameter regimes, while at other parameter values traveling waves exhibit either frontal or background instability. An analysis of the background steady states provided information about the spectrum of these waves. From an applications perspective, a two-dimensional collection of oscillators, corresponding to cells in the retina or in a layer of visual cortex, is most relevant, and for this reason we also considered some prototypical examples of two-dimensional evolution. In particular, we demonstrated that perturbed planar fronts can heal and resume their planar form, while radial fronts shrink. Our simulations of the system that initially has two fronts with bulging centers are in qualitative agreement with Carpenter's experimental observations [4] (see also the discussion of [5]).

The present work leads to numerous interesting questions for the further exploration of this and related systems. In particular, obtaining an analytical handle on the spectrum of the front in the co-traveling wave PDE and connecting this spectrum to the stability properties of the original ODEs would be extremely valuable from a theoretical perspective, not only in the context of the present setting but also for wide additional classes of lattice dynamical problems bearing traveling waves, such as generalized Frenkel-Kontorova (see [8] and references therein), Fermi-Pasta-Ulam 

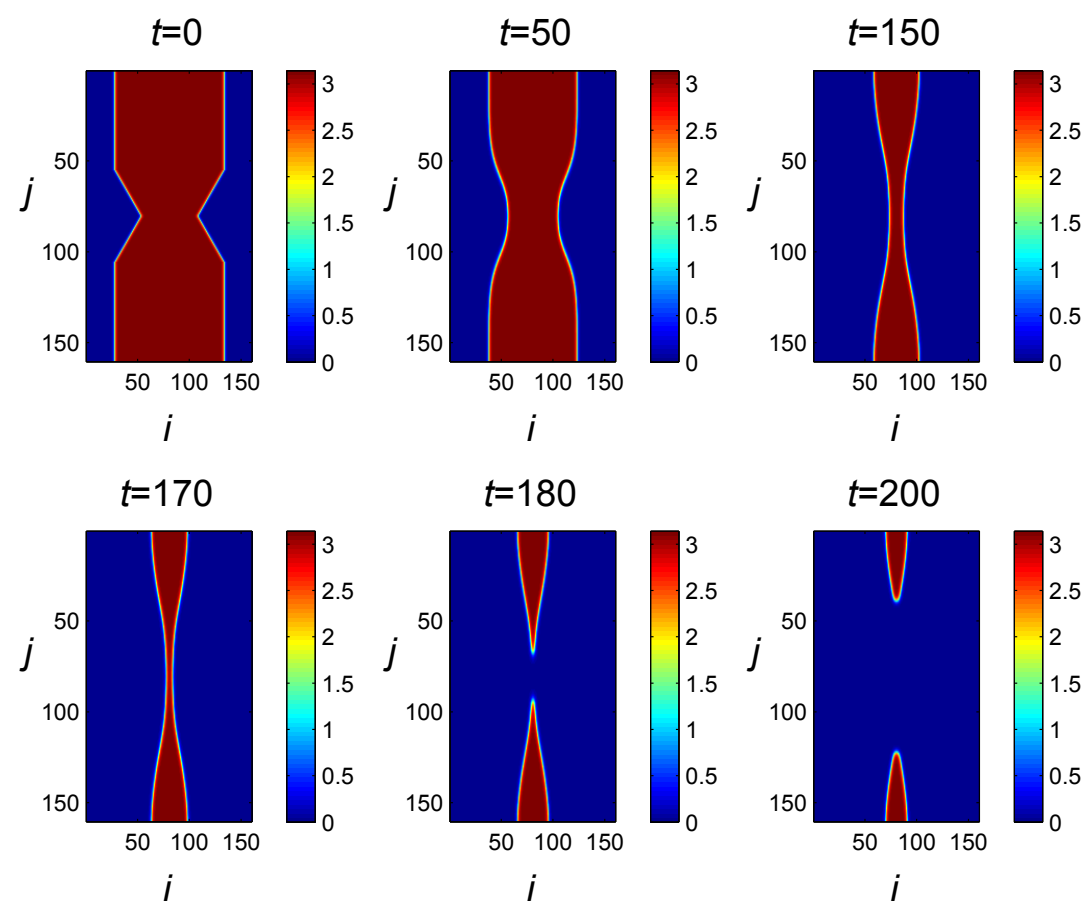

FIG. 20: Snapshots of two inwardly perturbed fronts at times $t=0,50,150,170,180$ and 200 when $k=2$, $\mu=0.15$. As in Fig. 18, the fronts meet near their centers, separate, and evolve into two (upper and lower) parts.

(see e.g. [9-11] and the discussion of Chapter 1 in [12]) or nonlinear Schrödinger systems (see e.g. [13-16] and the discussion of Chapter 21 in [17]). On the numerical front, while we explored a few prototypical cases of two-dimensional evolution, a better understanding of the stationary and traveling states in two dimensions clearly merits further investigation. Another interesting direction is to formulate and study solutions and spectra of a two-component problem that may support traveling pulses.

On the applications side, once the basic properties of this model system are more fully understood, it may serve as a form of computational test bed for exploring and making predictions about visual phenomena evoked by electrical stimulation. In this context, it may be interesting to explore the influence of changes in the amplitude or qualitative form of the forcing function on wave propagation [3] and to investigate waves induced by presenting traveling wave stimuli (e.g., [18, 19]), representing objects passing through the visual field, in addition to periodic forcing. Indeed, these adjustments may help resolve the model's current failure to capture the observation that neighboring fronts tend to propagate in a similar manner. At a more fundamental level, the present 
class of models appears to be a (modified) overdamped variant of the widely studied, so-called sine lattices (see e.g. [20] and, for a discussion of some of the relevant applications, [21]), hence it would be particularly interesting to explore hybrid variants of these models having as special case limits the overdamped and the undamped cases previously explored. Some of these issues are currently under investigation and will be reported upon in future publications.

\section{Acknowledgments}

P.G.K. gratefully acknowledges the support of the US-AFOSR under grant FA9550-12-1-0332, and the ERC under FP7, Marie Curie Actions, People, International Research Staff Exchange Scheme (IRSES-605096). P.G.K.'s work at Los Alamos is supported in part by the U.S. Department of Energy. JR was partially supported by the NSF award DMS-1312508.

\section{APPENDIX: SOME DETAILS ON NUMERICAL METHODS}

To solve the traveling wave Eq. (5), we used a second order forward difference scheme and sometimes a second order centered difference scheme to approximate the term $\phi^{\prime}(z)$. For a grid point $j$, these approximations are of the form $\left(-3 \theta_{j}+4 \theta_{j+1}-\theta_{j+2}\right) /(2 \Delta x)$ and $\left(\theta_{j+1}-\theta_{j-1}\right) /(2 \Delta x)$, respectively, where $\Delta x$ is the grid spacing. In some cases, the forward difference approximation used in solving Eq. (5) did not converge but the centered difference approximation did. Whichever approximation was used to solve Eq. (5) for the traveling wave $\phi(z)$, it was checked that the solution of the ODE system (1) with the initial condition $\theta_{i}(0)=\phi(i)$ produced results that were consistent with the obtained traveling wave solution.

In the following we analyze the stability of the background state in the discretized Eq. (5) with the forward and centered difference approximations described above. First, the forward difference approximation is analyzed. In Fig. 7, we see that the eigenvalues of the Jacobian for Eq. (5) approximate the eigenvalues of the continuum background as the number of points is increased, although the full structure of the forward difference spectral locus is more complex. The eigenvalues for the background can also be obtained in the context of the forward difference as follows. In this case, Eq. (6) obtained by linearizing Eq. (3) about the background equilibrium state $\Theta_{0}=0$ or $\Theta_{0}=\pi$ is replaced by

$$
\begin{aligned}
& V_{\tau}-c\left[\frac{-V(J+2, \tau)+4 V(J+1, \tau)-3 V(J, \tau)}{2 \Delta x}\right]= \\
& \quad k \cos (\mu)[(V(J+q, \tau)-2 V(J, \tau)+V(J-q, \tau))]-2 V(J, \tau),
\end{aligned}
$$




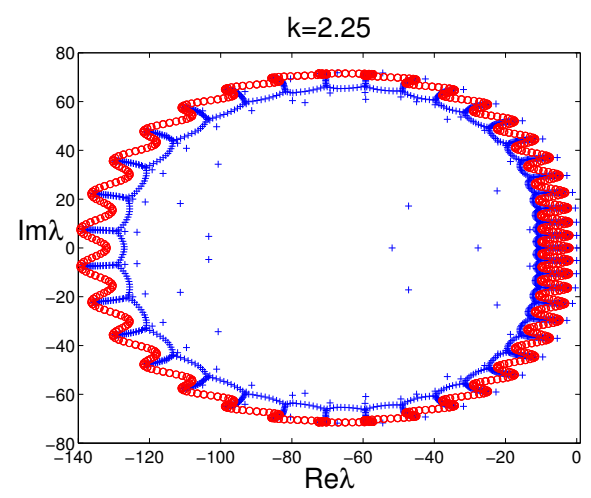

(a)

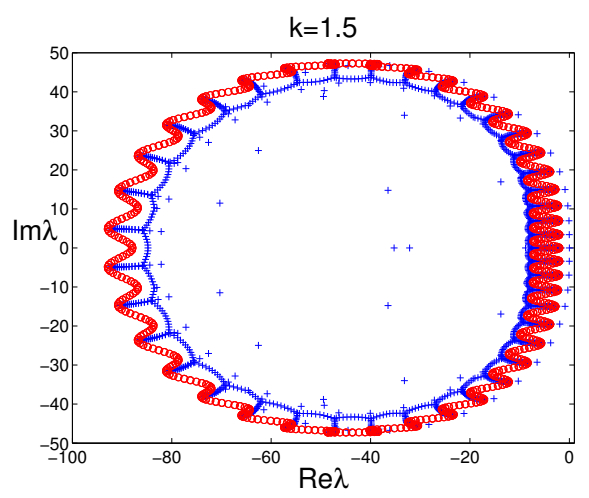

(b)

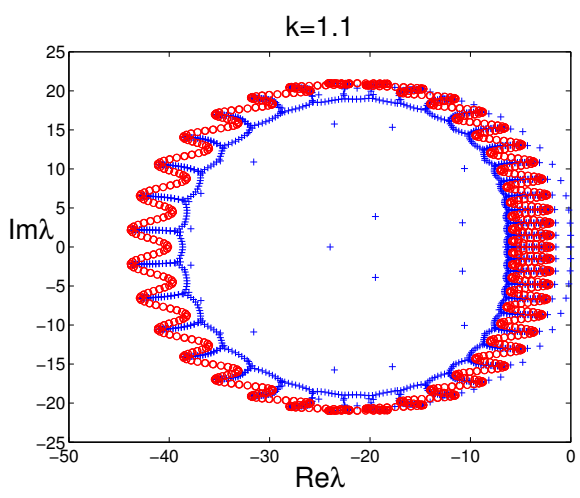

(c)

FIG. 21: Plot of the eigenvalues of the Jacobian associated with the linearization of Eq. (3) about the traveling wave solution (blue pluses), solved on [-25, 25] using 2001 nodes and a forward difference scheme, and the eigenvalues for the background equilibrium state (red circles) given by Eq. (12). Here $\mu=0.5$ and $k=2.25,1.5$ and 1.1 .

where $V(J, \tau)$ is the approximation of $v(z, \tau)$ at a grid point $z_{J}=J \Delta x$ for integer $J$ and $q$ is an integer such that $q \Delta x=1$. Seeking solutions in the form $V_{J}(\tau)=e^{\lambda \tau} e^{i J p \Delta x}$, where $p$ is the wave number, and solving for $\lambda$, we find that

$\lambda=c\left[\frac{-\cos (2 p \Delta x)+4 \cos (p \Delta x)-3}{2 \Delta x}\right]+2 k \cos \mu(\cos (p)-1)-2+i c\left[\frac{-\sin (2 p \Delta x)+4 \sin (p \Delta x)}{2 \Delta x}\right]$.

The real and imaginary parts of these eigenvalues parametrized by $p$ are shown by red circles in Fig. 21 at $k=2.25,1.5$ and 1.1 and $\mu=0.5$. For comparison, the eigenvalues of the Jacobian associated with the traveling wave solution are shown by blue pluses (recall also Fig. 7, where these eigenvalues are shown for the case $k=1.5$ and $\mu=0.5$ for different numbers of nodes in the discretization.) To solve (5), 2001 nodes are used in $[-25,25]$. The plots show that the two sets of eigenvalues are close to each other. 
Expanding Eq. (12) in Taylor series at small $\Delta x$, we obtain

$$
\lambda=-\frac{c p^{4}(\Delta x)^{3}}{4}+2\{k \cos (\mu)(\cos (p)-1)-1\}+i\left(c p+c \frac{p^{3}}{3}(\Delta x)^{2}+O\left((\Delta x)^{4}\right)\right),
$$

which yields Eq. (7) in the limit $\Delta x \rightarrow 0$. The principal part of the error in the real and imaginary parts is $-c \frac{p^{4}}{4}(\Delta x)^{3}$ and $c \frac{p^{3}}{3}(\Delta x)^{2}$ respectively. As the wave number $p$ increases, this error pushes the real part of $\lambda$ to $-\infty$ and the imaginary part to $+\infty$ and $-\infty$, again in line with the observations of Fig. 7.

It is interesting to explore the spectral properties of the background of the traveling wave using a centered difference approximation instead of the forward difference approximation. Eq. (11) is then replaced by

$$
V_{\tau}-c \frac{V(J+1, \tau)-V(J-1, \tau)}{2 \Delta x}=k \cos (\mu)(V(J+q, \tau)-2 V(J, \tau)+V(J-q, \tau))-2 V(J, \tau),
$$

and the eigenvalues $\lambda$ are given by

$$
\lambda=2 k \cos (\mu)(\cos (p)-1)-2+i \frac{c \sin (p \Delta x)}{\Delta x} .
$$

In this case there is no error in the real part of $\lambda$.

Generally, this centered difference approximation is numerically unstable for the advection equation given by (3); see [22]. Here, we find that the nonlinear term does stabilize it for large enough values of $k$, but the instability in the numerical method is observed for smaller values of $k$ even though the solutions of the traveling wave equation are stable, according to the forward difference approximation. For example, in Fig. 22 the eigenvalues obtained using the centered difference approximation of Eq. (5) are presented for $k=1.1,1.5$ and 2.25 with $\mu=0.5$. The real parts of the eigenvalues mostly lie between -5.86 and -2 for $k=1.1$, between -7.27 and -2 for $k=1.5$ and between -9.90 and -2 for $k=2.25$, which agree almost exactly with the continuum background theory based on Eq. (7). According to Fig. 22, however, with fixed $\mu=0.5$, the centered difference approximation predicts that traveling waves become unstable somewhere between $k=2.25$ and $k=1.5$, as some eigenvalues emerge with positive real part due to the instabilities associated with the centered difference approximation. In the results presented in the manuscript, care has been taken to avoid such spurious instabilities induced by the numerical scheme.

[1] L. Cervetto, G.C. Demontis, C. Gargini, British J. Pharmacol., 150, 383 (2007).

[2] E. Zrenner, Science. 295, 1022 (2002). 


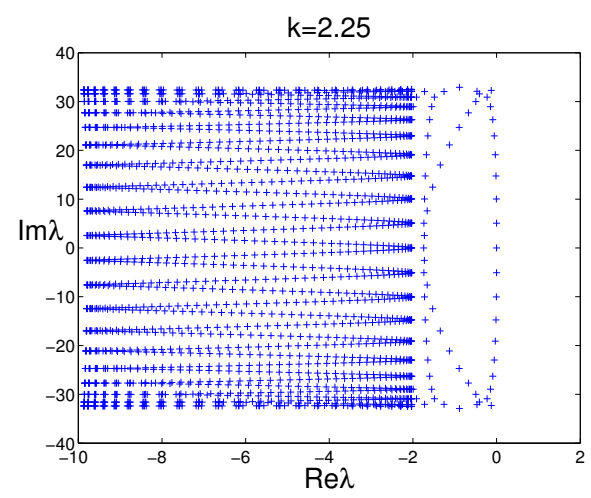

(a)

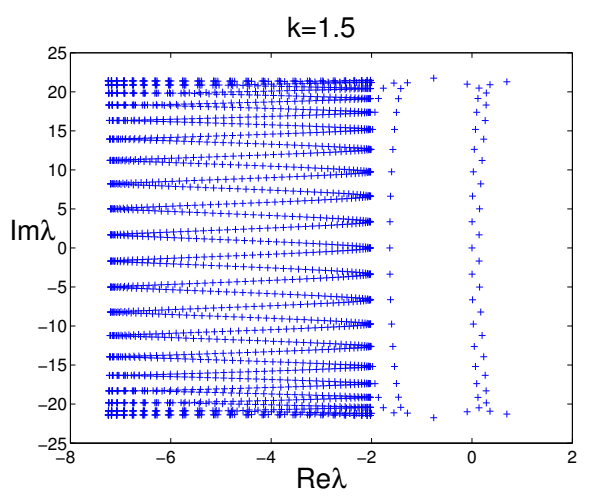

(b)

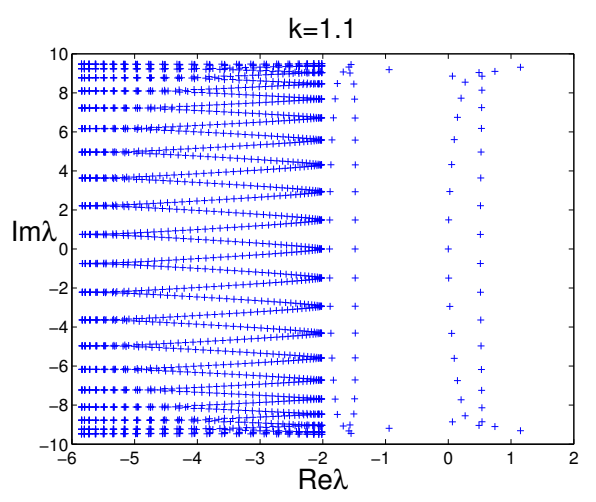

(c)

FIG. 22: Plot of the eigenvalues of the Jacobian associated with the linearization of Eq. (3) about the traveling wave solution (blue pluses), solved on [-25, 25] using 2001 nodes and centered difference approximation. Here $\mu=0.5$ and $k=2.25,1.5$ and 1.1 .

[3] E.J. Tehovnik, W.M. Slocum, Neurosci. \&3 Biobehav. Reviews, 37, 803 (2013).

[4] R. Carpenter, J. Physiol. 229, 767 (1973).

[5] J. Drover, G. Ermentrout, SIAM J. Appl. Dyn. Syst. 5, 529 (2006).

[6] H. Parks, G. Ermentrout, J. Rubin, Physica D 240, 554 (2011).

[7] B. Ermentrout, Simulating, analyzing and animating dynamical systems: a guide to XPPAUT for researchers and students, SIAM, Philadelphia, PA (2002).

[8] O. M. Braun and Y. S. Kivshar. The Frenkel-Kontorova model: concepts, methods and applications. Springer, Berlin-Heidelberg, 2004.

[9] G. Friesecke and R.L. Pego, Nonlinearity 12, 1601 (1999); G. Friesecke and R.L. Pego, Nonlinearity 15, 1343 (2002); G. Friesecke and R.L. Pego, Nonlinearity 17, 207 (2004). G. Friesecke and R.L. Pego, Nonlinearity 17, 2229 (2004).

[10] R.L. English and J.M. Pego, Proceedings of the AMS 133, 1763 (2005).

[11] A. Stefanov and P. Kevrekidis, Nonlinearity 26539 (2013).

[12] V. F. Nesterenko, Dynamics Of Heterogeneous Materials. (Springer-Verlag, New York, 2001). 
[13] D.E. Pelinovsky and V.M. Rothos, Phys. D 202, 16 (2005); D.E. Pelinovsky, Nonlinearity 19, 2695 (2006).

[14] O. F. Oxtoby and I. V. Barashenkov Phys. Rev. E 76, 036603 (2007).

[15] T.R.O. Melvin, A.R. Champneys, P.G. Kevrekidis and J. Cuevas, Phys. Rev. Lett. 97, 124101 (2006); see also Physica D 237, 551 (2008).

[16] M. Syafwan, H. Susanto, S.M. Cox and B.A. Malomed, J. Phys. A: Math. Theor. 45, 075207 (2012).

[17] P.G. Kevrekidis, The discrete nonlinear Schrödinger equation: mathematical analysis, numerical computation and physical perspectives, Springer-Verlag (Heidelberg, 2009).

[18] S.E. Folias and P.C. Bressloff, SIAM J. Appl. Math., 65, 2067 (2005).

[19] G.B. Ermentrout, J. Jalics, and J. Rubin, SIAM J. Appl. Math., 70, 3039 (2010).

[20] S. Homma and S. Takeno, Progr. Theoret. Phys. 72, 679 (1984); S. Takeno and S. Homma, Progr. Theoret. Phys. 7, 548 (1987).

[21] F. Zhang, Physica D 110, 51 (1997).

[22] K.W. Morton and D.F Mayers, Numerical Solution of Partial Differential Equations, Cambridge: Cambridge University Press, 1994.

[23] The speed of the front is numerically calculated as follows. We choose an integer grid point $k$ and place the right-traveling front to its left, near an integer grid point $m$ with $m<k$ such that $\left|\theta_{m}-\frac{\pi}{2}\right|<\left|\theta_{j}-\frac{\pi}{2}\right|$ for all $j$. Then $\theta$ is close to $\frac{\pi}{2}$ at the integer grid point $m$ than at any other integer grid point. The front is allowed to evolve using the standard fourth order Runge-Kutta method. We count the number of time steps $p$ such that $\left|\theta_{k}-\frac{\pi}{2}\right|<\left|\theta_{j}-\frac{\pi}{2}\right|$ for all $j$. The total time where $\theta=\frac{\pi}{2}$ lies in the interval $\left[k-\frac{1}{2}, k+\frac{1}{2}\right]$ is denoted as $p \Delta t$ where $\Delta t$ is the Runge-Kutta time step, and the approximation to the speed is then $\frac{1}{p \Delta t}$. The error is observed to be $O(\Delta t)$. If $p=0$, meaning that the front never entered the interval $\left[k-\frac{1}{2}, k+\frac{1}{2}\right]$, then the speed is zero, assuming that the method was allowed to run long enough. 